Portland State University

PDXScholar

$12-6-2019$

\title{
HF IQ Mixer VFO Temperature Compensation and Drive Level Optimization for Opposite Sideband Suppression
}

Katlin Anne-Rostomyan Dahn

Portland State University

Follow this and additional works at: https://pdxscholar.library.pdx.edu/open_access_etds

Part of the Electrical and Computer Engineering Commons Let us know how access to this document benefits you.

\section{Recommended Citation}

Dahn, Katlin Anne-Rostomyan, "HF IQ Mixer VFO Temperature Compensation and Drive Level Optimization for Opposite Sideband Suppression" (2019). Dissertations and Theses. Paper 5431.

https://doi.org/10.15760/etd.7304

This Thesis is brought to you for free and open access. It has been accepted for inclusion in Dissertations and Theses by an authorized administrator of PDXScholar. Please contact us if we can make this document more accessible: pdxscholar@pdx.edu. 
HF IQ Mixer VFO Temperature Compensation and Drive Level Optimization for Opposite Sideband Suppression

by

Katlin Anne-Rostomyan Dahn

A thesis submitted in partial fulfillment of the requirements for the degree of

\author{
Master of Science \\ in \\ Electrical Engineering
}

Thesis Committee:

Richard Campbell, Chair

Branimir Pejcinovic

Martin Siderius

Robert Bass

Portland State University

2019 


\begin{abstract}
An amateur radio $40 \mathrm{~m}$ band temperature compensated variable frequency oscillator (VFO) with drive-level optimized for opposite sideband suppression for use as the local oscillator (LO) of a high frequency (HF) IQ image reject mixer is considered in this work. The first problem of this thesis was the discovery of the HF IQ mixer's opposite sideband suppression dependence on LO drive-level. As a solution to this problem, the author built a fixed drive-level VFO for the optimal opposite sideband suppression. This then led to the second problem of this thesis; the discovery by this author of the well-known problem of VFO instability in the particular case where the frequency drift primarily arises from ambient temperature variation. This historically rich and technically challenging problem illustrates the difference between an actual high performance 40m JFET Hartley VFO, and an academic textbook schematic. The textbook circuit with standard components was abysmally drifty, and this thesis demonstrates application of theory to reduce the drift to $-4.25 \mathrm{ppm} /{ }^{\circ} \mathrm{C}$ and $+8.5 \mathrm{ppm} /{ }^{\circ} \mathrm{C}$ for the $40 \mathrm{~m}$ frequency ranges: $7.05-7.22 \mathrm{MHz}$, and $6.95-7.13 \mathrm{MHz}$, respectively. Drift was also reduced to $-1.42 \mathrm{ppm} /{ }^{\circ} \mathrm{C}$ with styrofoam packaging on tank components for the frequency range of 7.05- 7.22 MHz. The author constructed a temperature chamber to perform the temperature compensation tests, which is described in this work. The use of boiling water to relieve mechanical stress in the VFO components was investigated in this work, and measured results are presented. The iterations of measurements, compensation calculations, and the final achieved drift are also described in this work. Above $40 \mathrm{~dB}$ opposite sideband suppression from the HF IQ mixer was achieved with the frequency stabilized VFO of this work, designed for optimized LO drive-level in the range of $+2.25-+5 \mathrm{dBm}$. Additionally, within this optimized LO drivelevel range, opposite sideband suppression levels of above $50 \mathrm{~dB}$ were achieved.
\end{abstract}




\section{Dedication}

To my loving and supportive husband, Narek. To my wonderful parents. To my sister and greatest friend, Chelsea. To my Grandparents and wonderful Aunts and Uncles. 


\section{Acknowledgements}

Acknowledgement is due to my adviser, professor, and mentor Professor Richard Campbell, KK7B. I am forever grateful to Dr. Campbell for sparking my interest in amateur radio; the HAM radio community is a source of inexhaustible knowledge and open-source experience and resources. Although I am by no means the only student who has the great privilege to say this; it has been an honor to be Dr. Campbell's student.

I would like to acknowledge Wes Hayward, W7ZOI, from whom this work has benefited greatly from personal correspondences and from his many published and un-published works.

I would like to thank Phillip Wong, Dr. Donald Duncan, and Dr. Douglas Hall all who were instrumental in my undergraduate years. Phillip gave me the opportunity to teach and Dr. Duncan gave me the opportunity tutor students. The counsel of time and experience are indeed invaluable, but perhaps less without the special few who offer attention and sincere guidance to highlight their significance; I thank Dr. Hall for his thoughtful observations and kind advice.

I would also like to acknowledge the ladies who process all master's theses for Portland State University; this great feat falls on the shoulders of two women, Brenda Fugate and Andrea Haack. I'd like to acknowledge Andrea Haack who despite her large workload allowed me to pull up a chair and benefit from her invaluable experience. 


\section{Table of Contents}

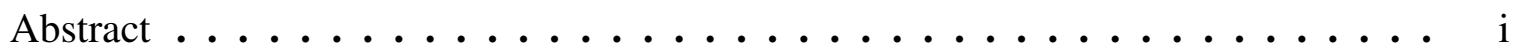

Dedication ........................ ii

Acknowledgements .................... iii

List of Tables $\ldots \ldots \ldots \ldots \ldots \ldots \ldots \ldots \ldots \ldots \ldots$

List of Figures $\ldots \ldots \ldots \ldots \ldots \ldots \ldots \ldots \ldots \ldots \ldots$ vi

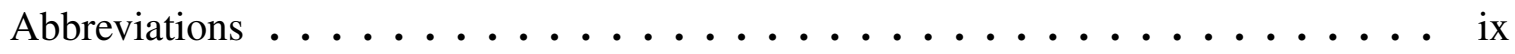

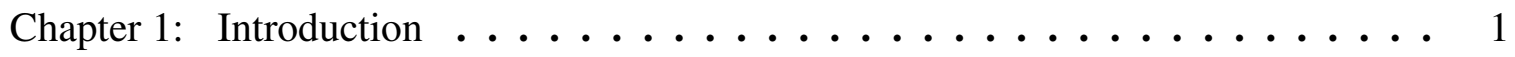

1.1 Receiver Architectures . . . . . . . . . . . . . . . . . 2

1.2 Frequency Converters . . . . . . . . . . . . . . . . . . 6

1.3 Organization of Thesis $\ldots \ldots \ldots \ldots \ldots$

Chapter 2: IQ Crystal Set $\ldots \ldots \ldots \ldots \ldots \ldots \ldots \ldots \ldots \ldots \ldots$

Chapter 3: Enhancements to the IQ Crystal Set: IQX2 $\ldots \ldots \ldots \ldots \ldots$

Chapter 4: Half Frequency VFO . . . . . . . . . . . . . . 15

Chapter 5: Measurements and Discoveries . . . . . . . . . . . 19

Chapter 6: VFO Frequency Drift $\ldots \ldots \ldots \ldots \ldots \ldots \ldots$

Chapter 7: Conclusion and Future Work $\ldots \ldots \ldots \ldots \ldots$

References......................... 48

Appendix A: Mixer Mathematics . . . . . . . . . . . . . . . . . 49

A.1 Terminology . . . . . . . . . . . . . . . . . . . . . . 49

A.2 Baseband I \& Q Output . . . . . . . . . . . . . . . . . . . . . . 49

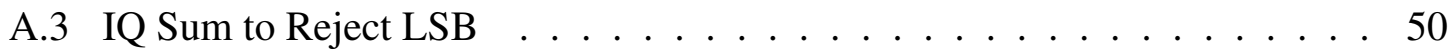

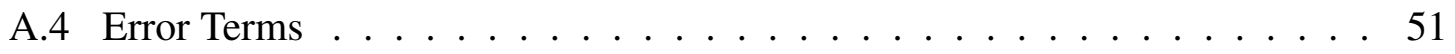

A.5 Upper Sideband (USB) desired for low-side LO injection: $\omega_{R F}>\omega_{L O} \ldots 51$

A.6 Lower Sideband (LSB) image for low-side LO injection: $\omega_{R F}<\omega_{L O} \ldots 53$

A.7 Trigonometric Identities . . . . . . . . . . . . . . . . . . . 54

A.8 IQ Image Rejection Spectra Diagrams $\ldots \ldots \ldots \ldots \ldots \ldots$

Appendix B: Additional Receiver Background . . . . . . . . . . . . . . . 59 


\section{List of Tables}

5.1 The iqx2 opposite sideband suppression with VFO utilizing variable coupler to vary LO drive-level. . . . . . . . . . . . . . . . . . . 22

5.2 The iqx2 opposite sideband suppression with temperature compensated VFO utilizing the test setup of Figure 5.0.7. 4.7pF non-NP0 ceramic capacitor used for coupler on JFET gate. . . . . . . . . . . . . . . . 26

6.1 The tank components for calculation of the TCL of the inductor, and for measurements of Figures 6.0.7, 6.0.8, and 6.0.10 . . . . . . . . 33

6.2 Tank components for fitting the mathematical model. $\mathrm{NP} 0-0 \mathrm{ppm} /{ }^{\circ} \mathrm{C}$ and SM for silver mica, Figure 6.0.11 . . . . . . . . . . . . . 36

6.3 7.05 - 7.22 $\mathrm{MHz}$ tank components, $\mathrm{NP0}-0 \mathrm{ppm} /{ }^{\circ} \mathrm{C}$ and $\mathrm{SM}$ for silver mica. Figures 6.0.13 and 6.0.12, and Equation 6.0.7 . . . . . . . . . . . . 38

6.4 6.95 - 7.13 MHz tank components, $\mathrm{NP0}-0 \mathrm{ppm} /{ }^{\circ} \mathrm{C}$ and $\mathrm{SM}$ for silver mica. Figure 6.0.14 . . . . . . . . . . . . . . . . . . 40 


\section{List of Figures}

1.1.1 Simple Superheterodyne receiver with single IF stage. . . . . . . . . . . 3

1.1.2 Simple direct conversion Receiver. . . . . . . . . . . . . . . 4

1.1.3 direct conversion Receiver with RF amp and IF port filtering. . . . . . . . 5

1.2.1 Unbalanced JFET mixer [33]. . . . . . . . . . . . . . . . . . . 6

1.2.2 Singly balanced JFET mixer [7]. . . . . . . . . . . . . . . . . . . 7

1.2.3 Double-balanced diode ring mixer. . . . . . . . . . . . 8

2.0.1 Hartley IQ Image Reject Mixer for SSB . . . . . . . . . . . . . . 11

2.0.2 IQ mixer with single SPDT switch [2] . . . . . . . . . . . . . . 12

3.0.1 The IQX2 with frequency doubler and LO-RF offset derivation. . . . . . . 13

4.0.1 JFET Hartley VFO schematic. . . . . . . . . . . . . . 15

4.0.2 3.5 MHz VFO built on a microR2 original prototype board [27]. . . . . . 16

4.0.3 JFET Hartley VFO schematic with 80m (3.5 MHz) component values. . . 17

4.0.4 JFET Hartley VFO spectrum with $+3.86 \mathrm{dBm}$ output power with $3 \mathrm{~dB}$ attenuator on output. HD2 $-28.5 \mathrm{dBc}$ and HD3 $-24 \mathrm{dBc}$. . . . . . . . . . 17

5.0.1 The iqx2 opposite sideband suppression signal generator test setup. . . . . 19

5.0.2 The iqx2 opposite sideband suppression sensitivity to LO drive-level with the signal generator test setup of Figure 5.0.1 at 3.5 MHz LO. . . . . . . . 20

5.0.3 The iqx2 opposite sideband suppression sensitivity to LO drive-level and frequency with the signal generator test setup of Figure 5.0.1 RF input level fixed at $-60 \mathrm{dBm}$. . . . . . . . . . . . . . . 21 
5.0.4 The iqx 2 opposite sideband suppression test setup with signal generator for RF input and VFO for local oscillator (LO). . . . . . . . . . . . 22

5.0.5 Equivalent JFET small-signal model (left) with coupling capacitor on the gate forming voltage divider with $C_{g s} \ldots \ldots \ldots \ldots \ldots$

5.0.6 Phase noise of VFO measured with R\&S FSWP. . . . . . . . . . . . . 24

5.0.7 The iqx2 opposite sideband suppression signal generator test setup with $3 \mathrm{~dB}$ attenuator to provide $50 \Omega$ input between VFO and the iqx 2 input. . . 25

6.0.1 Temperature Chamber for temperature compensation tests [14] $\ldots \ldots 28$

6.0.2 On the two ends are electrolytic capacitors, in the center is a film capacitor, and the remaining two are class 2 ceramic capacitors. . . . . . . . . 29

6.0.3 First is a silver mica capacitor, the following 4 are class 1 ceramic capacitors of different temperature coefficients. . . . . . . . . . . . . . 29

6.0.4 VFO drift with initial parts available from junk box. . . . . . . . . . 30

6.0.5 VFO warmup drift with initial parts available from junk box, $12.2 \mathrm{kHz}$ in 45 min and climbing! . . . . . . . . . . . . . . . 30

6.0.6 VFO drift after changing a few key components in the tank circuit. . . . . 31

6.0.7 VFO temperature drift after 1 st annealing of inductor $-63.8 \mathrm{ppm} /{ }^{\circ} \mathrm{C} . \quad \ldots \quad 32$

6.0.8 VFO temperature drift after 2 nd annealing of inductor $-46.94 \mathrm{ppm} /{ }^{\circ} \mathrm{C} . \quad \ldots 33$

6.0.9 Boiling of entire circuit in pot of water on the stove to anneal inductor. . . 34

6.0.10 Comparison of warmup of oscillator before and after annealing one and two times. . . . . . . . . . . . . . . . 35

6.0.11 Overcompensation with $56 \mathrm{pF}$ N750 compensating capacitor. . . . . . . 37

6.0.12 VFO drift with temperature compensating capacitor and low, (zero) tempco capacitors for frequency doubled tuning range 7.05 - 7.22 MHz, (doubled frequency). . . . . . . . . . . . . . . . . . . 39 
6.0.13 Temperature drift of 7.05 - 7.22 MHz, (doubled frequency), tuned oscillator with styrofoam packaging on tank inductor and capacitors, -1.42

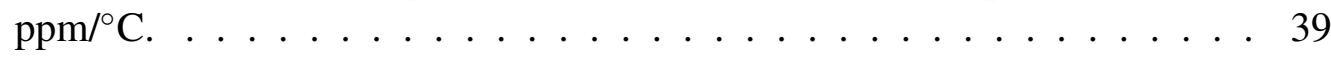

6.0.14 VFO drift with temperature compensating capacitor and low, (zero) tempco capacitors for frequency doubled tuning range $6.95-7.13 \mathrm{MHz}$, (doubled frequency). . . . . . . . . . . . . . . . . . . 40 
ARRL American Radio Relay League

ASP Analog Signal Processing

BB Baseband

BFO Beat Frequency Oscillator

BPF Band Pass Filter

C0G EIA standard temperature coefficient of capacitance code: $0 \mathrm{ppm} /{ }^{\circ} \mathrm{C}+/-$ $30 \mathrm{ppm} /{ }^{\circ} \mathrm{C}$

CW Continuous Wave

DSB Double Sideband

DSP Digital Signal Processing

DUT Device Under Test

EIA Electronic Industries Association

EMRFD Experimental Methods in RF Design

HF High Frequency

IF Intermediate Frequency

IP2 Intermodulation Product 2nd Order

IP3 Intermodulation Product 3rd Order

IIP3 Input-referred Intermodulation Product 3rd Order

IQ In-phase \& Quadrature

IQX2 In-phase \& Quadrature with Frequency Doubler (X2)

JFET Junction Field-Effect Transistor

KK7B Dr. Campbell's Amateur Radio Call Sign

KJ7DMF Katlin Dahn's Amateur Radio Call Sign

LO Local Oscillator 


\begin{tabular}{|c|c|}
\hline LNA & Low Noise Amplifier \\
\hline LPF & Low Pass Filter \\
\hline LSB & Lower Sideband \\
\hline NP0 & $\begin{array}{l}\text { Industry standard temperature coefficient of capacitance: negative } 0 \\
\mathrm{ppm} /{ }^{\circ} \mathrm{C}\end{array}$ \\
\hline $\mathbf{N} 470$ & $\begin{array}{l}\text { Industry standard temperature coefficient of capacitance: negative } 470 \\
\mathrm{ppm} /{ }^{\circ} \mathrm{C}\end{array}$ \\
\hline PCB & Printed Circuit Board \\
\hline $\mathbf{R F}$ & Radio Frequency \\
\hline SFDR & Spurious-Free Dynamic Range \\
\hline SG & Signal Generator \\
\hline SM & Silver Mica \\
\hline SNR & Signal to Noise Ratio \\
\hline SSB & Single Sideband \\
\hline TRF & Tuned Radio Frequency \\
\hline USB & Upper Sideband \\
\hline VFO & Variable Frequency Oscillator \\
\hline VHF & Very High Frequency \\
\hline W7EL & Roy Lewallen's Amateur Radio Call Sign \\
\hline W7ZOI & Wes Hayward's Amateur Radio Call Sign \\
\hline
\end{tabular}




\section{Chapter 1 \\ Introduction}

The focus of the present work is on two specific blocks of a direct conversion radio receiver for an amateur license portion of the High Frequency (HF) band of the electromagnetic spectrum which is defined as 3-30 $\mathrm{MHz}$ in frequency corresponding to $100-10 \mathrm{~m}$ in wavelength. The first block examined is a novel single single-pole-double-throw (SPDT) switch In-phase and Quadrature (IQ) image-reject mixer with frequency doubler module [1], (referenced hereafter as the "iqx2"), designed for $7 \mathrm{MHz}, 40 \mathrm{~m}$, operation and for use in a direct conversion receiver. The second receiver block examined is a Junction Field-Effect Transistor (JFET) Hartley Variable Frequency Oscillator (VFO) operating at half the receiver design frequency, 3.5 MHz. The VFO was modified from the 40m microR2 receiver VFO [27], for use as the Local Oscillator (LO) input for the iqx2 mixer module.

The iqx 2 mixer was presented to the author for this work as an experimental module. The predecessor to the iqx2 was carefully measured and published in an IEEE paper [2], but the iqx 2 investigated here in this work has not been subjected to such rigorous testing and this thesis work is the first publication using the iqx2. The experimental improvements to the IEEE SPDT iq mixer [2] resulting in the iqx2 were done by the author's professor, they were not performed by the author of this work. Instead, the author performed opposite sideband suppression measurements as a function of LO drive-level and frequency. These measurements revealed opposite sideband suppression sensitivity to LO drive-level, and the author decided to solve this sensitivity by building a fixed drive-level VFO to maximize opposite sideband suppression performance of the iqx 2 .

Construction of the VFO lead to the discovery of a historically rich and interesting problem of frequency instability. From the point of this discovery, frequency stabilization of the VFO became the chief focus of this work. The frequency drift was reduced through use of 
inductor (L) and capacitor (C) tank components with temperature drift coefficients which oppositely drifted with temperature to cancel one another. This temperature coefficient cancellation significantly minimized frequency drift due to ambient temperature variations. The author employed an iterative and heuristic frequency compensation technique [14] to achieve frequency drift with temperature of: $-1.42 \mathrm{ppm} /{ }^{\circ} \mathrm{C}$ with component packaging, and $-4.25 \mathrm{ppm} /{ }^{\circ} \mathrm{C}$ without packaging. The author built the temperature chamber test equipment, used in this work to achieve frequency stability, which is described in chapter 6. Lewallen [28] suggested that boiling the VFO inductor to "anneal" it would improve the frequency drift characteristics. It was inconvenient to remove the inductor in this work, and removal would have introduced new construction stress. A different method, therefore, was used in this work: the entire VFO circuit was immersed in boiling water. Careful measurements of frequency drift showed significant improvement in frequency drift after 5 minute and then 10 minute immersions. The drift measurements of the 5 and 10 minute boiling of the VFO are presented in chapter 6 of this work. After the VFO was frequency stabilized, the author applied it back to its original purpose-to optimize the iqx 2 opposite sideband suppression with an optimal fixed LO drive-level. Above $40 \mathrm{~dB}$ opposite sideband suppression was achieved for $\mathrm{LO}$ drive-levels $+2.25-+5 \mathrm{dBm}$ for variation in power supply from 4.2-4.5 V. Above $50 \mathrm{~dB}$ was achieved for $\mathrm{LO}$ drive-levels in this range for various RF input levels, Table 5.2.

\subsection{Receiver Architectures}

The oldest radio communications receiver architectures were comprised of a tuned circuit and single detector which would capture the audio envelope of the transmitted Amplitude Modulated (AM) signal, to be heard with high impedance headphones. This sort of receiver with radio frequency (RF) tuning stages is a so-called Tuned Radio Frequency receiver (TRF) an example of which is the "cat's whisker" receiver, Figure B.0.1 in appendix 
B, which used only the power supplied from the transmitted signal. The receiver LC circuit would be tuned to the carrier frequency, governed by Equation B.0.1, either by tunable capacitor and/or tunable inductor. The crystal, such as galena, with a lever-adjustable wire contact would behave as a primitive diode - allowing conduction in one direction - rectifying the incoming analog AM signal to capture the modulated envelope of the carrier to be heard in the earphone.

TRF receivers later included amplification and complex tuning to broaden the receive sensitivity and bandwidth, aided with the invention of vacuum tubes, more stable diodes, and creatively complex ganged filter-amplifier tuning stages. Along with vacuum tube receivers came regenerative and superregenerative receivers utilizing the oscillatory, highgain instability of vacuum tube amplification stages. The principle of heterodyning offered a way to convert received signal frequencies into a different, locally fixed and controlled, frequency which reduced the number of ganged-tuning stages required as most selectivity and signal amplification could then be achieved at a fixed frequency. Methods of heterodyning are discussed in section 1.2.

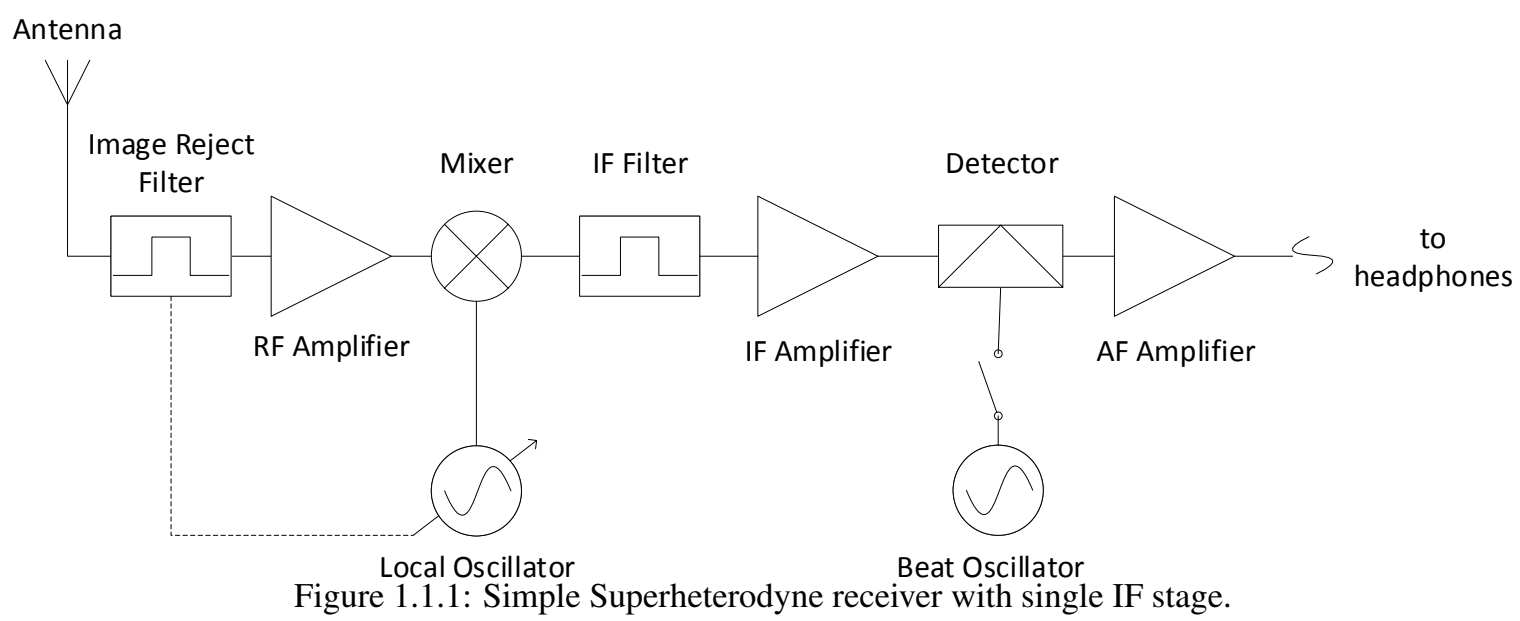

The superheterodyne, superhet, architecture employs use of what is called an intermediate frequency (IF) that is higher than the final baseband converted signal frequency. The superhet architecture acheives the final baseband signal in at least two frequency conver- 
sions. In Figure 1.1.1 two conversions are shown with the second oscillator, "Beat Oscillator", (also known as beat frequency oscilltor (BFO)), as part of the "detector" block which is another mixer. For AM detection the beat oscillator would be disconnected and the audio envelope demodulated in the mixer, much like the TRF example. For single sideband (SSB) or continuous wave $(\mathrm{CW})$ the beat oscillator is connected and performs conversion of the IF signal to another lower signal, typically audio.

The superhet has the capability of truly outstanding performance in selectivity and dynamic range, but acquisition of such performance involves many stages of filtering, amplification and clever choice of IF frequencies. A superhet for high performance can quickly become quite complex. Additionally the mulitiple frequency conversions can have a marked effect on the quality of the received signal, and each desired freqeuncy band requires its own frequency conversion plan [30] [6]. One side-effect of the multiple conversions in a supherhet is that it is susceptible to internally generated spurs, a.k.a. birdies, generated from interaction between the internal LOs and unwanted frequency products.

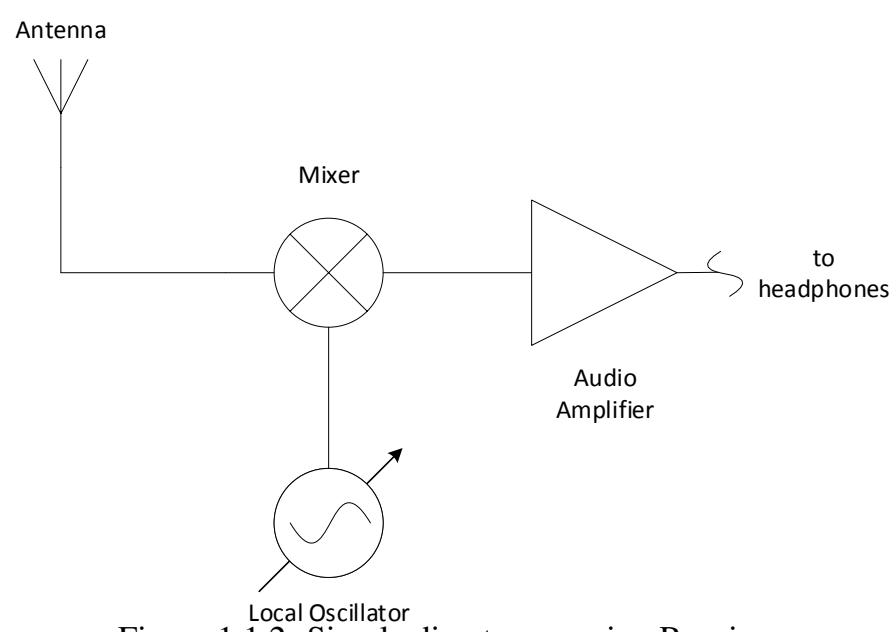

Figure 1.1.2: Simple direct conversion Receiver.

A type of receiver architecture which employs a single conversion is called a direct conversion receiver. Homodyning - zero-IF-is frequently referred to as direct conversion, but direct conversion can also apply to a low audio frequency IF, $0 \mathrm{~Hz}$ to $2 \mathrm{kHz}$ or so. 
In fact this audio output is not an intermediate frequency, but the final baseband, but this terminology is a hold-over from superheterodyne terminology. Figure 1.1.3 shows a simple and typical direct conversion receiver.

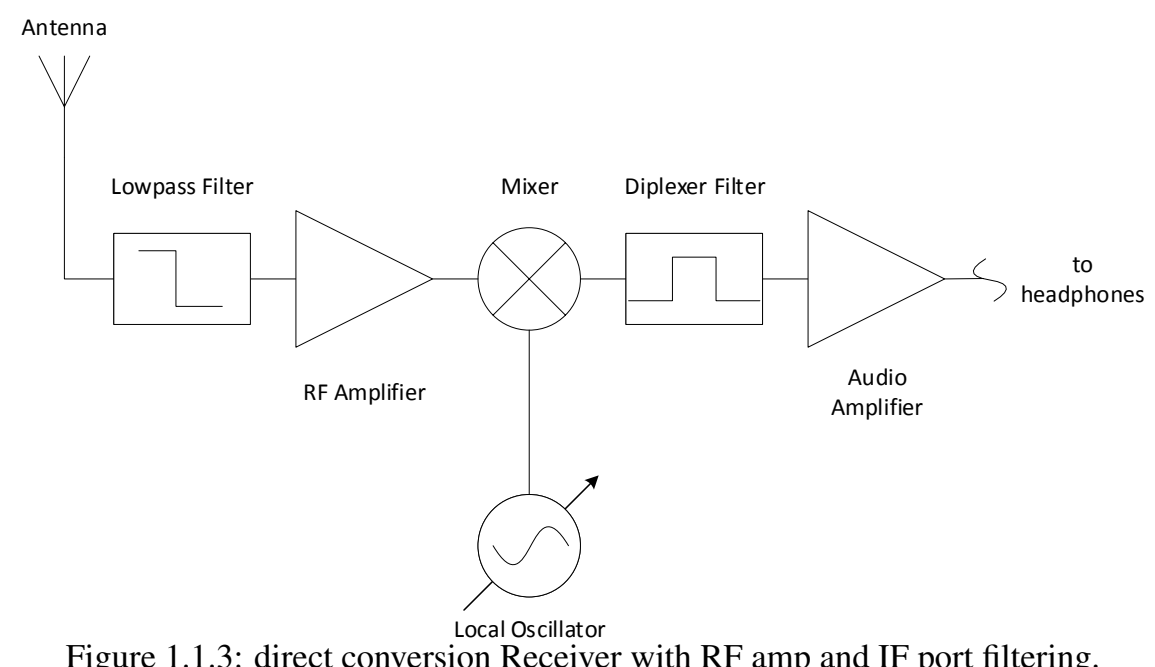

Figure 1.1.3: direct conversion Receiver with RF amp and IF port filtering.

A benefit of direct conversion is that because the signal only undergoes a single conversion the signal integrity can be much better than the superhet case. The direct conversion does not have multiple LOs and conversion stages and therefore it has no internally generated audio pitches, "birdies". The spurious-free dynamic range (SFDR) of direct conversion receivers can be much higher than the superhet. Additionally it can be seen in Figures 1.1.3 and 1.1.2 that the direct conversion architecture is simpler than the superhet, Figure 1.1.1.

Direct conversion receivers are not, however, immune to limitations. One of which is that its LO is either exactly the same frequency as the input RF signal, or only an audio frequency-range distance away. This makes it possible for any leaked or re-radiated LO energy to become incident on the antenna and be indistinguishable from the desired signal thereby corrupting it. There are solutions to get around this all of which involving shielding of the LO signal. LO leakage is particulalry problematic in direct conversion receivers because it can serve to re-enforce itself. Receiver corruption happens because LO energy 
at the RF port mixes to produce a DC product, see appendix A and section 1.2, which imbalances the mixer and allows more LO energy to leak to the RF port. See section 1.2 and reference [4] for more discussion of mixer balance. Another limitation is that direct conversion receivers are very sensitive to second-order non-linearities, which are distortion products that can show up right in the receive band near or at DC.

\subsection{Frequency Converters}

The frequency converter is the block of a receiver and transmitter often referred to as the mixer. Its basic function for heterodyning is to convert a signal from one frequency to another either higher, (up-conversion), or lower, (down-conversion), frequency. The way this is achieved is by multiplying two signals and using the simple trigonometric identity in Equation A.7.1 to acquire two output terms at the sum, (up-conversion), and difference, (down-conversion), frequencies of the two input signals.

The presence of the input signals' frequencies, RF and LO, at the output, IF, depends on the balance of the mixer. An unbalanced mixer will have both the input signals' frequencies present on the output; a single-balanced mixer will cancel one of the input frequencies on the other ports; and a double-balanced mixer will cancel both input frequencies on the other ports. The use of circuitry duplication, symmetry, and ideal matching of components contributes to the cancellation of RF, LO, and/or IF signals at the other ports [4]. Though, any deviations from these idealities leads to some feedthrough.

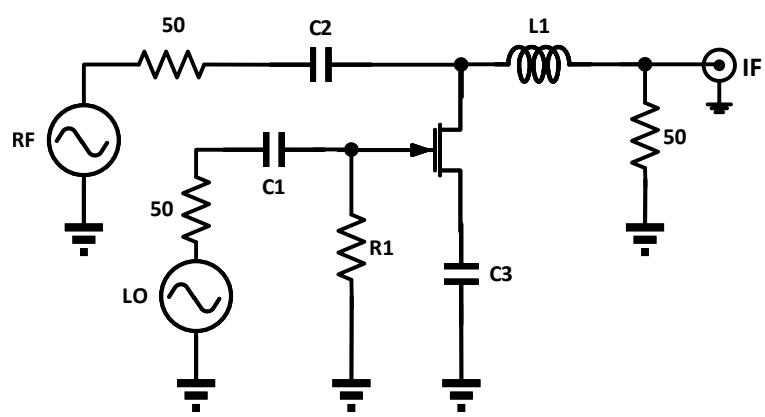

Figure 1.2.1: Unbalanced JFET mixer [33]. 
Figure 1.2.1 is an example of an unbalanced mixer using a single JFET [33] [4]. RF and LO signals are applied to the drain and gate, respectively. Low pass filtering at the drain output passes the IF. But if the RF and LO signals are still able to feedthrough to the output and other ports, proper filtering on each port is needed to reduce them. The $\mathrm{X}$-band mixer in [33] by Steven Maas utilizes coupled line and stub filters on the ports to suppress other port frequencies. This filtering is shown in lumped-element form in Figure 1.2.1 as a diplexer on the drain. In Figure 1.2.2, Figure 1.2.1 has been duplicated and the LO signal applied differentially to the gates of the two JFETs. The IF output is differential through a transformer, but because the RF input is single-ended, applied in parallel to the drains of the FETs. The differential application of LO nulls its frequency at the RF port [4]. Conversely, RF energy at the LO port is cancelled through the transformer null. RF to IF isolation is improved as the RF is single-ended and the IF taken differentially, but the LO will be present at the IF output.

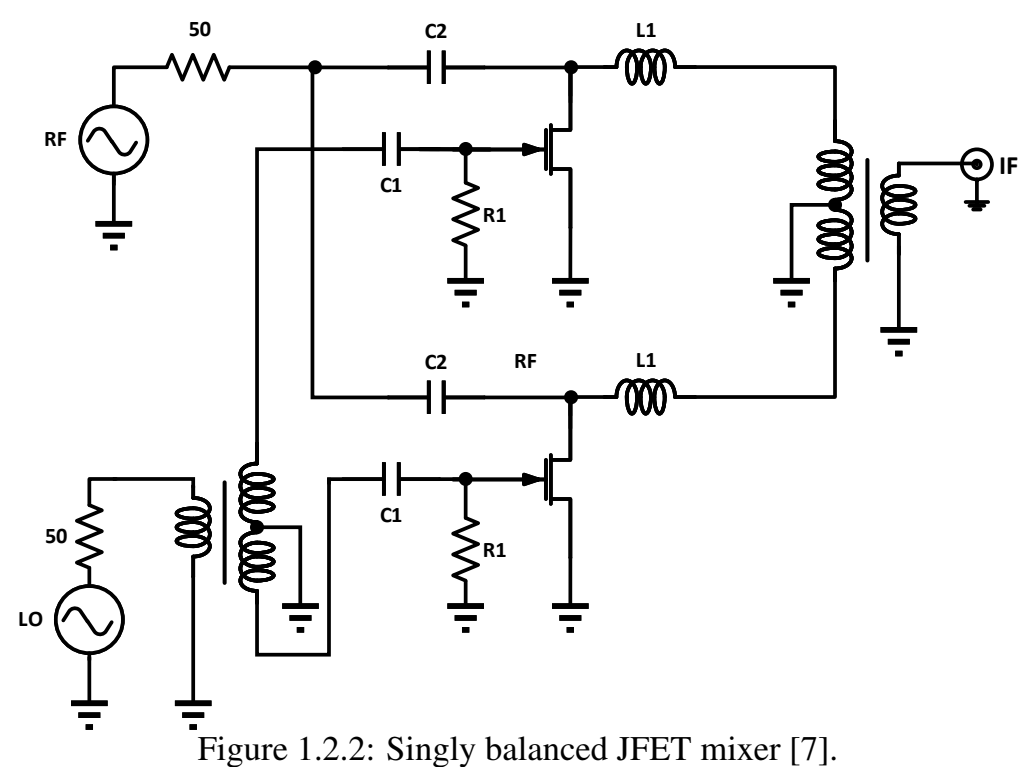

An example of a doubly-balanced mixer is the diode ring mixer where both LO and RF are cancelled at the IF port through symmetry, Figure 1.2.3. Care is needed in terminating the IF mixer port with a wideband $50 \Omega$ load to maintain balance. References [4] 
and [25] give discussion of proper termination techniques. A diplexer on the IF port is a popular termination strategy, which KK7B has employed in many designs. There is no transformer impedance transformation that occurs from the mixer IF port to the RF port [5] and therefore proper wideband $50 \Omega$ termination at the IF port translates to $50 \Omega$ at the RF.

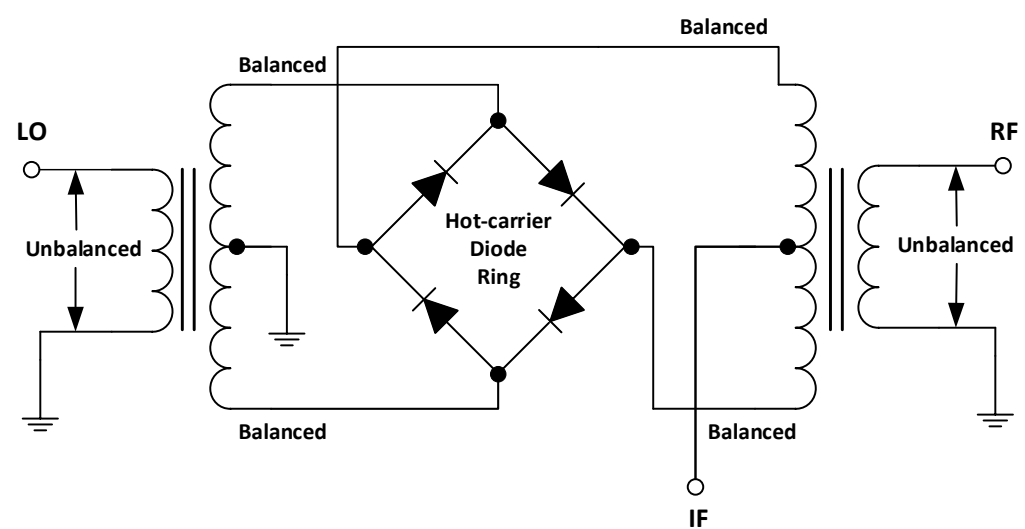

Figure 1.2.3: Double-balanced diode ring mixer.

The passive JFET mixer, both singly-balanced and un-balanced, offers very good performance in IIP3 due to high linearity and can have very low noise-figures [33] [34]. The FET mixer, however, suffers from low 2nd order distortion (IP2) [2], whereas the diode ring mixer shows good IP2 performance though its noise Figure and IIP3 performance are often not as good as its JFET counterpart. Because 2nd order linearity is so important for direct conversion receivers and the conversion loss between the passive FET mixer and the diode ring are comparable, the diode ring was chosen as the candidate for the iqx2 [2].

Additionally, active mixers like the ubiquitous Gilbert cell mixer are not typically top choices for high performance direct conversion receivers [30]. This is because the mixer in a direct conversion application comes before any significant channel selectivity and would therefore apply amplification to desired and undesired signals alike. Minimal amplification is therefore presented before the mixer in a direct conversion receiver, with the exception of a LNA with good reverse isolation, which is important for typical receiver noise Figure definition as well as improved LO-RF isolation. 
In low-IF applications, the image channel is separated by two times the IF frequency. In many practical cases, this is too close to the desired channel to be effectively filtered out, especially if large signals are present in the image band. In low-IF cases it is possible, with very sharp filters, to remove the image band from the receiver. However, they are very difficult to achieve at frequencies above $50 \mathrm{MHz}$, not to mention there is excess loss on desired weak signals. An alternative to filtering that can offer good performance is called a phasing method discussed in great detail in EMRFD chapter 9 [6]. The idea of phasing is similar to what happens in the balanced cases of the mixers mentioned, signal cancellation happens through combining signals with opposite phase. A mathematical and visual frequency domain explanation of phasing in receivers is summarized in appendix A.

With phasing, the limitation of the steepness of filters' out of band rejection and the frequency limitations of those filters is eliminated. Theoretically the phasing method is capable of achieving perfect image-rejection, though practically $30-40 \mathrm{~dB}$ of image rejection is routinely observed [30] due to device mismatches. The IQ mixer is a good choice for direct conversion applications to reduce/reject the image and therefore reduce noise inflicted on the received signal.

\subsection{Organization of Thesis}

The progression of this report by chapter number will be as follows: (1) Receiver architectures and the present direct conversion architecture; Frequency conversion methods and the present double-balanced diode-ring IQ method; (2) description of the IQ mixer module first version; (3) Enhancement of the IQ module to the present version, iqx2, explored in this work; (4) Integration of the half-frequency VFO for fixed LO drive-level; (5) Measurement, discoveries and improvements; iqx2 measurements, the first problem of LO drive-

level sensitivity; VFO initial measurements, the second problem of frequency driftiness and the source of the problem; (6) Exploration into solutions for VFO frequency drift, applica- 
tion of solution and resulting measured stability performance; (7) conclusion, summary of work done and further work towards a complete direct conversion receiver. 


\section{Chapter 2 \\ IQ Crystal Set}

The iqx 2 module was designed with key direct conversion receiver performance characteristics in mind. As mentioned direct conversion receivers for low-IF applications have an image that is very close to the desired band and thus difficult to filter. Superherterodyne receivers can suffer from this problem too, if the IF is too close to the desired band or if the band frequency is too high for high selectivity filters. Thus it is common for superhets to employ multiple conversion with a high first IF. The use of an in-phase, I, and a quadrature, $\mathrm{Q}$, representation of the input RF signal is useful in that the $90^{\circ}$ phase difference between I and Q channels can be used to destructively interfere with the undesired signal after the signals are converted and an additional $90^{\circ}$ has been applied to I or Q. The math explaining this can be found very comprehensively in Chapter 9 of EMRFD [6], a summary of which has been provided in appendix A along with a frequency-domain visual representation.

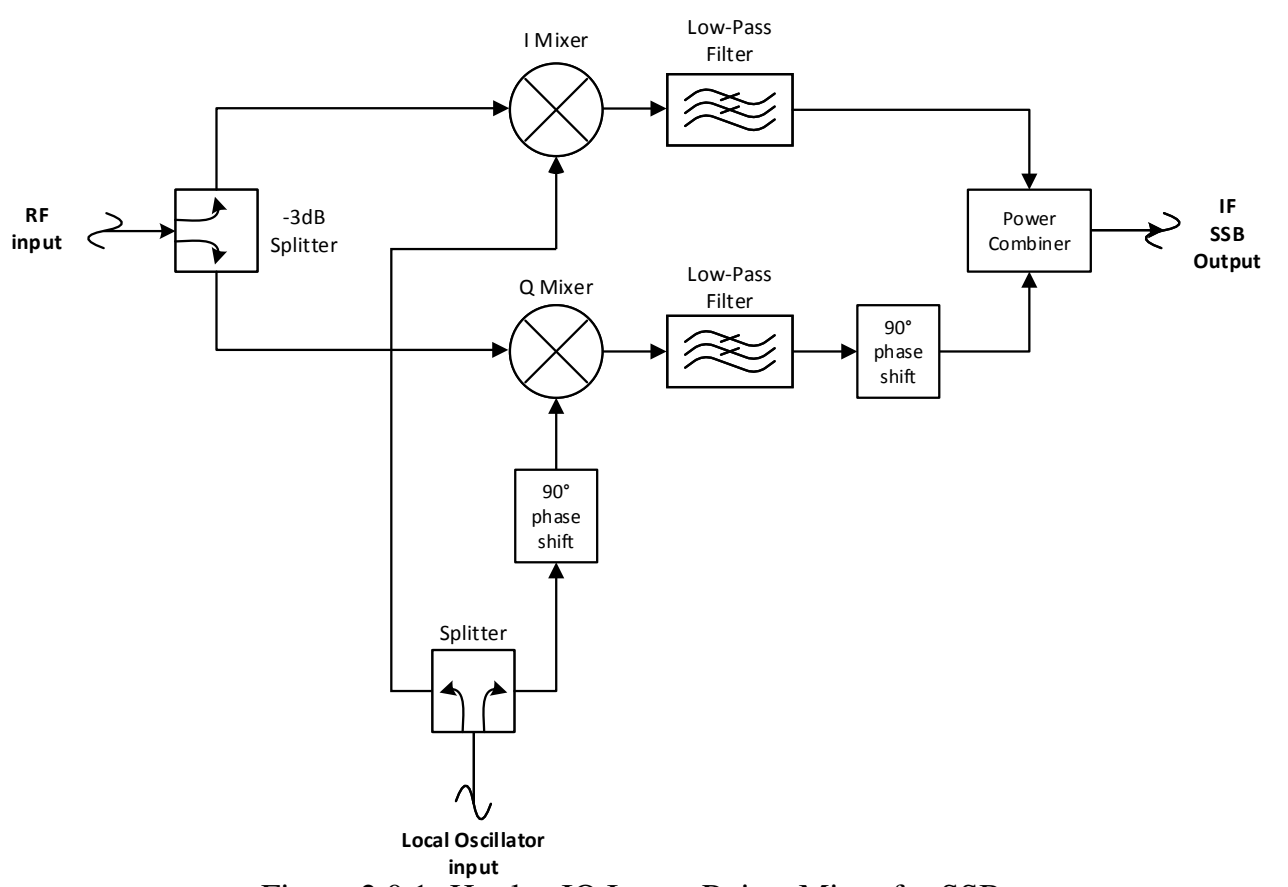

Figure 2.0.1: Hartley IQ Image Reject Mixer for SSB.

Figure 2.0.1 shows a generic block diagram of an IQ image-reject mixer with an I 
and a $\mathrm{Q}$ mixer and with quadrature placed on the LO signal, i.e. $90^{\circ}$ phase shift. For wideband applications it is useful to place quadrature on the LO, but for direct conversion applications the bandwidth is narrow and placing quadrature on the RF is also suitable. Narrow-bandedness allows dual purpose of the selectivity components in the signal paths in providing proper mixer port terminations [2].

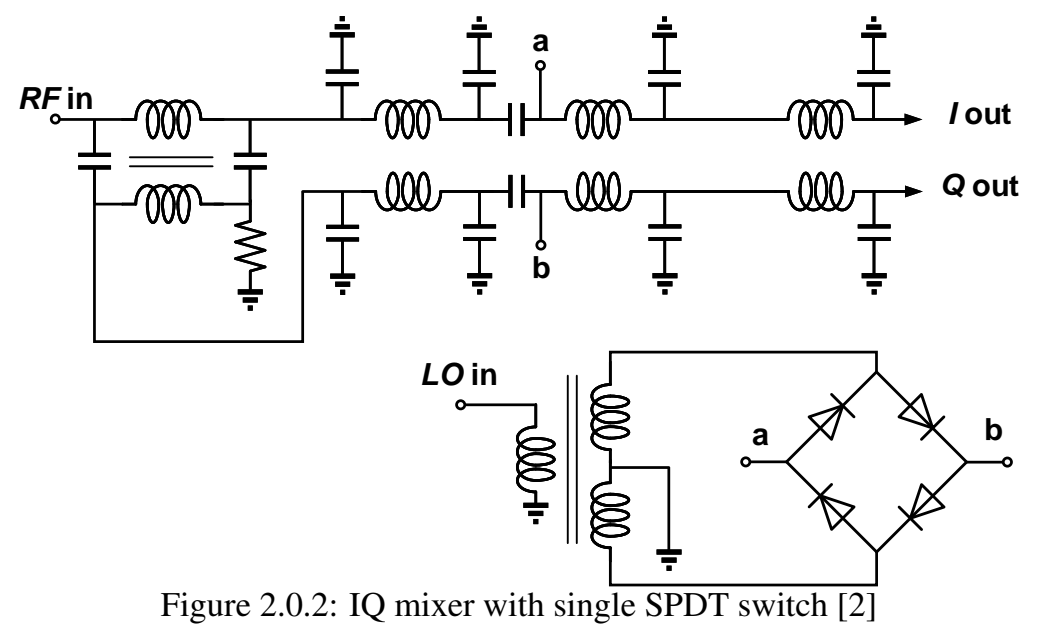

Figure 2.0.2 shows the scematic diagram of the IQ crystal set described in [2] for the diode ring mixer case. The interesting thing here is that a single single-pole-double-throw, SPDT switch as the mixer was used instead of the typical dedicated I and Q mixer seen in Figure 2.0.1. With quadrature on the RF path, the I and the Q are alternately connected to the mixer and to ground so the mixer works as if the I and Q had dedicated mixers. The article [2] also explored a singly-balanced MOSFET switch in place of the diode ring mixer, but measured second-order non-linearity performance was not as good as the diode ring nor the measured IQ stability. 


\section{Chapter 3 \\ Enhancements to the IQ Crystal Set: IQX2}

The iqx2 explored in this work sports a few enhancements from its previous predecessor: frequency doubling, and a LO-RF DC offset trim. The author's professor made these enhancements and a few cursory measurements on their performance before passing the module on to the author. The idea was for the author to make some measurements on the iqx2 and discover something for which to base a thesis on. The ensuing measurements and subsequent unfolding of this thesis's work is discussed in chapter 5. In this chapter, the general functioning and purpose of the iqx2 enhancements is discussed.
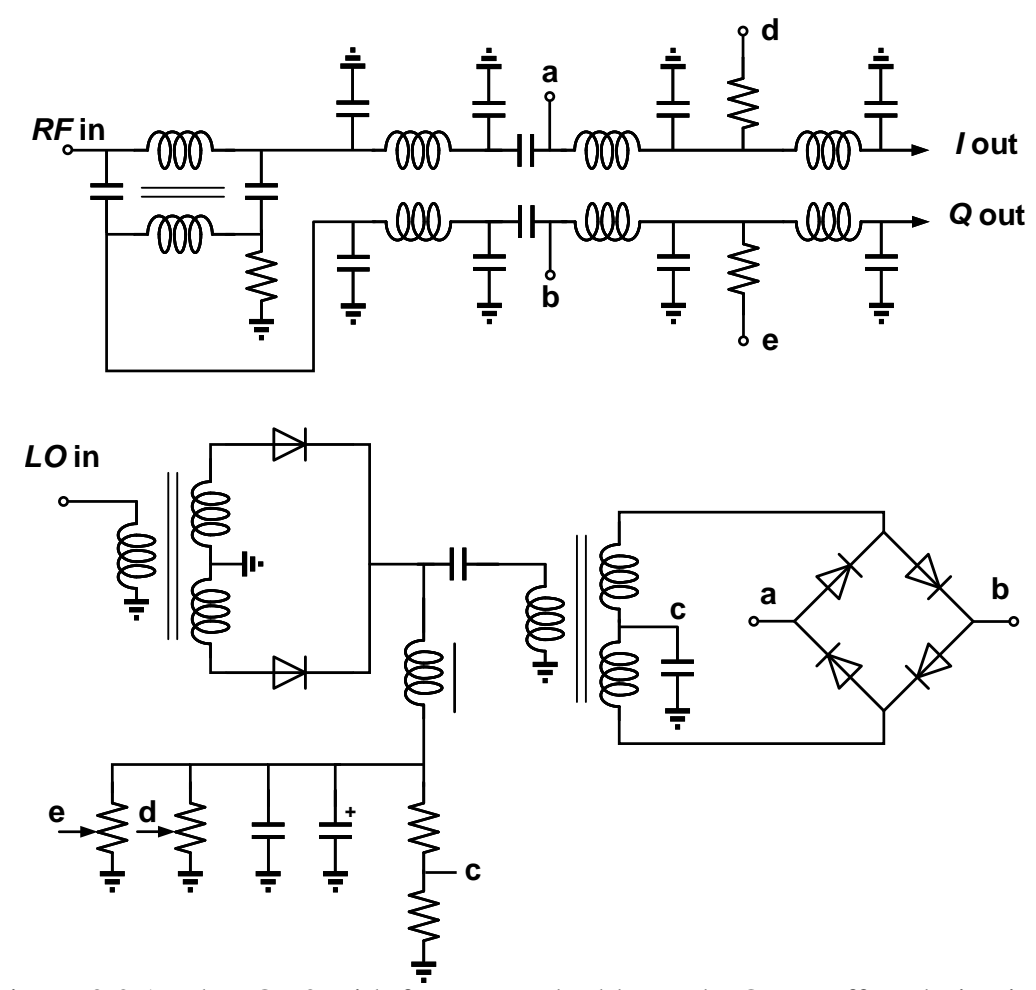

Figure 3.0.1: The IQX2 with frequency doubler and LO-RF offset derivation.

The addition of the frequency doubler is good practice especially for use in a direct conversion receiver to help against the LO leakage problem because it allows use of a LO frequency at half the operating frequency. Thus if the $\mathrm{LO}$ were able to leak out and become 
incident on the antenna, it would be far enough away to filter out. Additionally, any residual LO energy that made it to the RF port would not produce a DC output.

The self-mixing of the LO, and the ensuing DC output, does not carry any information to corrupt the desired signal, but will imbalance the mixer. This imbalancing action corrupts the natural cancellation of balanced signals at the mixer ports because the otherwise balanced signals have amplitude discrepancies from the DC. This consequently allows even more LO energy to appear at the RF port of the mixer, which has the ultimate undesired effect of degrading the opposite sideband suppression, as well as allowing AM demodulation from degraded 2nd-order non-linearity performance.

The other enhancement to the IQ crystal set was offset trim for the IQ signal paths to null the LO at the RF port. This can be seen in Figure 3.0.1 at points 'd' and 'e'. The frequency doubler produces a small amount of DC at the output of the frequency doubler at point "c", and this is used to derive trimmable DC offsets at the I and Q branches with potentiometers at points "d" and "e". This enhancement is also aimed at improving the LO-RF isolation and image-rejection (opposite sideabnd suppression). Residual LO at the RF port can be tuned out by tuning the DC offsets at I and Q. These I and Q DC trims could also be used to cancel a problematic AM signal frequency and thereby improve 2nd order distortion [1]. This would be done by applying an off-frequency AM signal at the RF port and tuning it out, at the output, with the DC offset trims [1]. 


\section{Chapter 4 \\ Half Frequency VFO}

The trimming IQ DC offset enhancement had an interesting side-effect of making the iqx2 opposite sideband suppression LO drive-level dependent [1]. This was interesting because, in contrast, the 2015 IQ crystal set with the diode-ring mixer—but without the frequency doubler-displayed very low variability with LO changes [2]. Measurements of this discovery are presented in chapter 5. This LO drive-level sensitivity was the first problem discovered in this work and lead to the construction of a fixed-level VFO at half frequency to stabilize the image-rejection performance. A classic Hartley oscillator was chosen as the VFO topology.

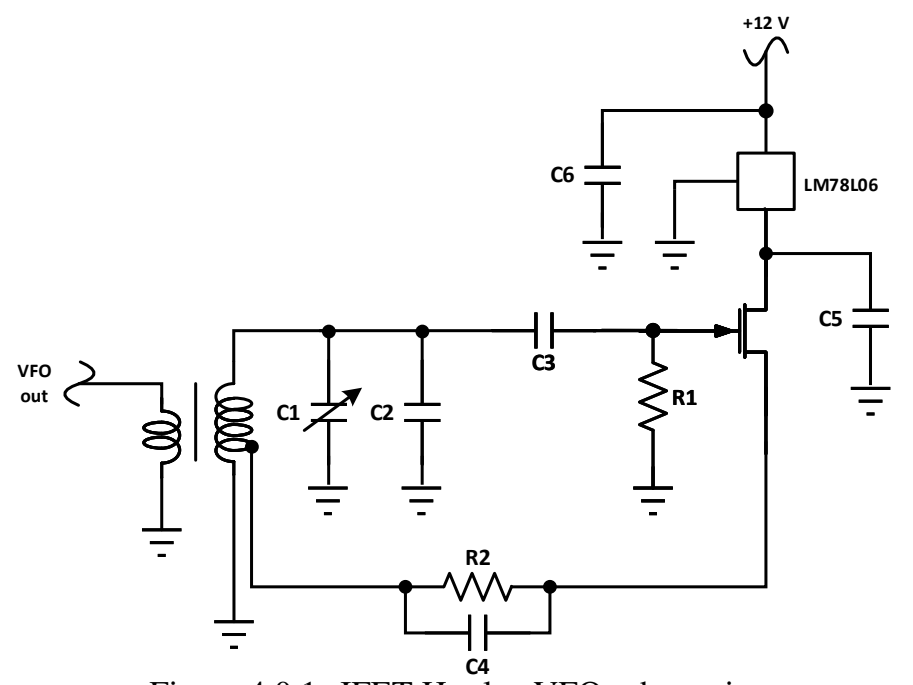

Figure 4.0.1: JFET Hartley VFO schematic.

The JFET Hartley VFO of Figure 4.0.1 is a classic design found in many HAM radio projects, not least those by W7EL, W7ZOI, and KK7B and found in many texts [4] [16] [22] and papers [27] [28] [29]. The microR2 40m version was taken as the base model for this work's LO and was built by the author on the original microR2 prototype board, Figure 4.0.2, of KK7B to serve as a benchmark. The microR2 [27] LO output was about $+10 \mathrm{dBm}$ at $7 \mathrm{MHz}$. Next the VFO was scaled to work for half the frequency, at $3.5 \mathrm{MHz}$. 


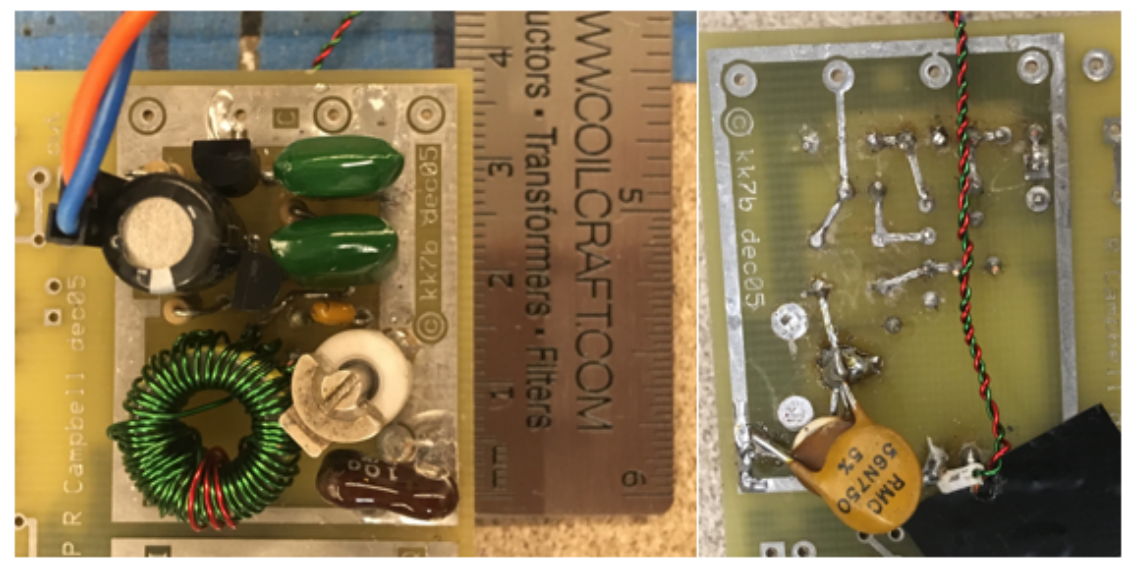

Figure 4.0.2: 3.5 MHz VFO built on a microR2 original prototype board [27].

At first, the author having already hand wound the tapped inductor on FT43-7 toroid for 7 $\mathrm{MHz}$ and fastened it into the board, thought to save time and re-use this inductor and scale the tank capacitor by $4 \mathrm{x}$ for the half frequency. This produced a non-oscillating circuit and further contemplation as to why suggested that there was too much capacitance to sustain oscillation. The Q of the LC tank can be roughly approximated as an RLC series circuit with the resistive losses assumed from the inductor, the $\mathrm{Q}$ to first order can be expressed in Equation 4.0.1. The oscillations were likely unable to start due to the lowering of the Q by about half when the capacitor was scaled up by 4 . Therefore a new tapped inductor, scaled up by 2 , was built and paired with capacitance, scaled up by 2 , and oscillations had no problem starting.

$$
Q=\frac{1}{R} \sqrt{\frac{L}{C}}
$$

The scaled oscillator, however, had a problem—frequency drift. This was observed on the spectrum analyzer as the signal swiftly marched off the screen. The author built the oscillator with available components from the author's junk box because, plagued by a myopic condition common to all novices—in a word, ignorance-the "NP0" and "COG" footnotes on the schematics did not have much meaning. This was until the observation 


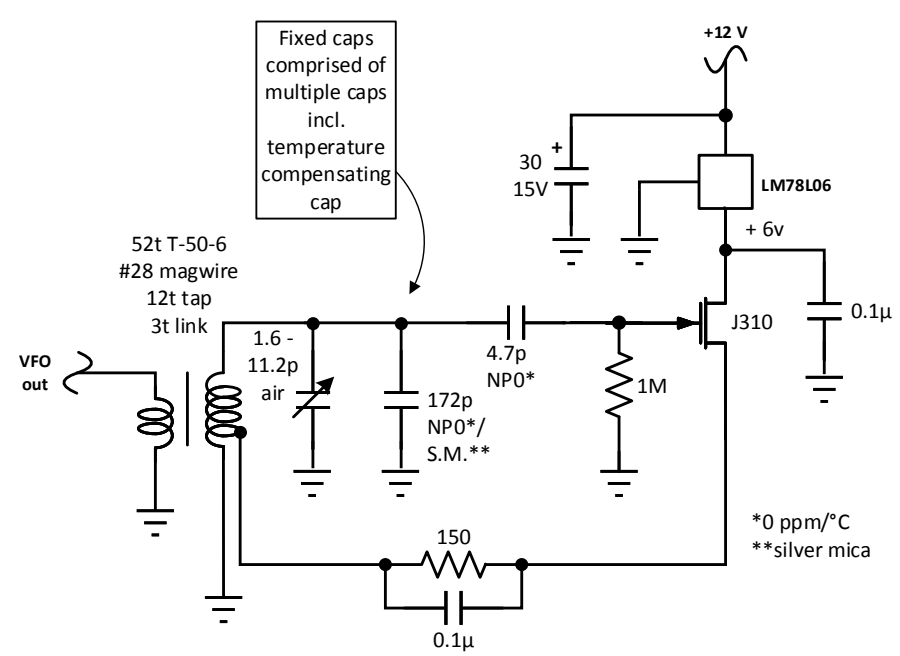

Figure 4.0.3: JFET Hartley VFO schematic with $80 \mathrm{~m}(3.5 \mathrm{MHz})$ component values.

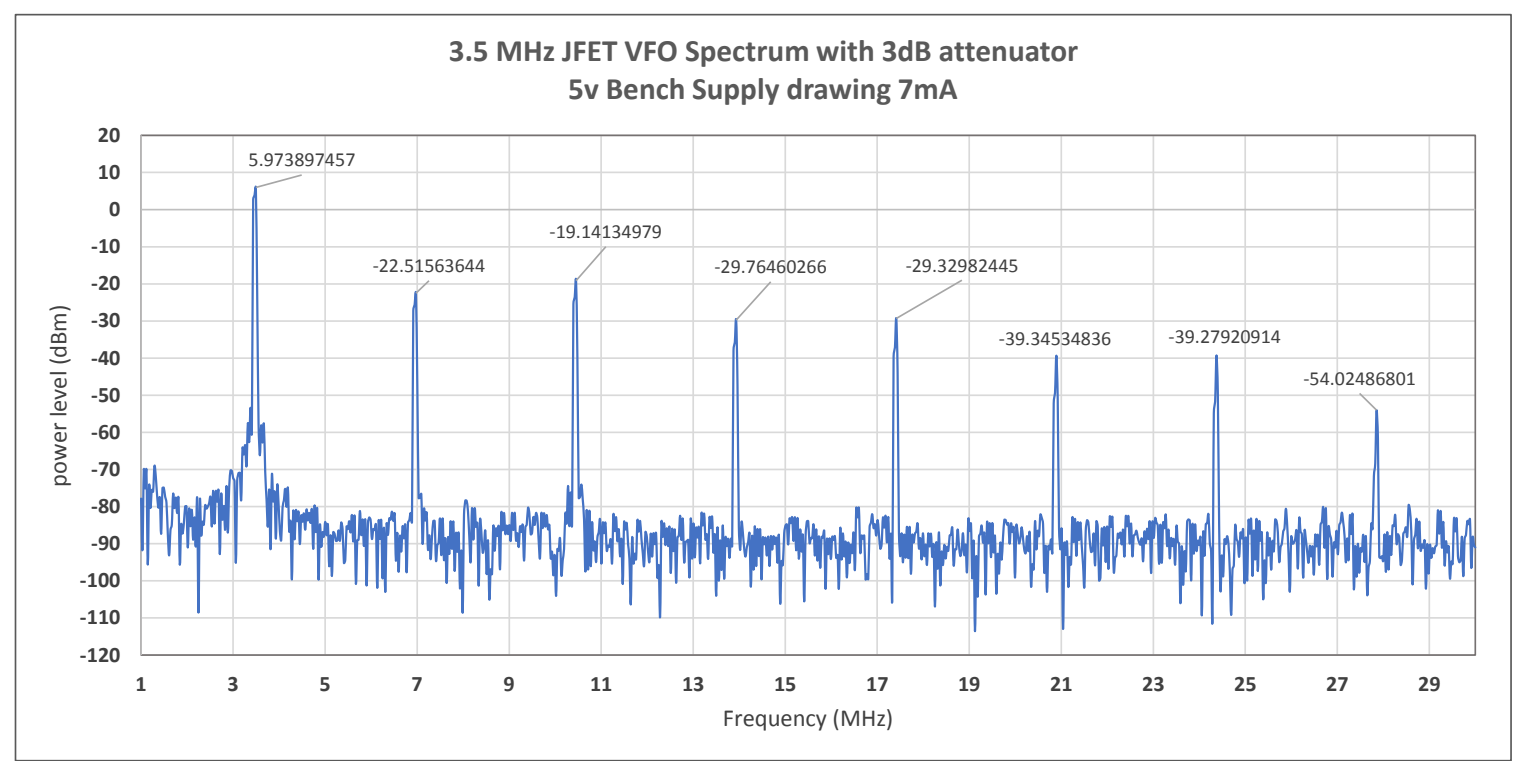

Figure 4.0.4: JFET Hartley VFO spectrum with $+3.86 \mathrm{dBm}$ output power with $3 \mathrm{~dB}$ attenuator on output. HD2 $-28.5 \mathrm{dBc}$ and HD3 $-24 \mathrm{dBc}$.

of the VFO's frequency drift, and a subsequent hunt for solutions to better understand and to solve the problem. Fortunately, oscillator frequency drift is a problem with lots of interesting history and significance in radio design, meaning there is ample literature on the subject. Radio receivers have a great concern for frequency stability of the local oscillator, especially for suppressed carrier SSB, because instability may render desired signals uncapturable, and/or audio receptions unpleasant or unintelligible. Of the few common 
culprits for frequency instability, ambient temperature variation-induced frequency drift is considered in this work. The temperature compensated circuit with component values is shown schematically in Figure 4.0.3, and photographically in Figure 4.0.2. The oscillator's output spectrum is shown in Figure 4.0.4. The method of achieving the frequency stability is discussed in chapter 6. Once the oscillator was built and stabilized, it was then put to use to solve the opposite sideband suppression LO drive-level sensitivity problem of the iqx2 module, which is discussed in chapter 5. 


\section{Chapter 5 \\ Measurements and Discoveries}

The initial measurements of the iqx 2 in this work, where we found that the opposite sideband suppression of the iqx2 module was LO drive-level dependent, are shown in Figure 5.0.2, using the test setup in Figure 5.0.1. As mentioned, this was an unintended side-effect of deriving the IQ LO-RF, (or off-frequency AM suppression), DC offset trim from the negative DC produced by the frequency doubler circuitry [1]. The LSB is the sideband being suppressed with the phasing IQ image reject mixer, iqx2. It is common for single sideband ( $\mathrm{SSB}$ ) receivers in the HF bands to suppress the lower sideband in favor of the upper sideband. Both sidebands were measured, first the USB $2 \mathrm{kHz}$ above twice the LO frequency and then the RF signal generator (SG) was tuned $4 \mathrm{kHz}$ down through zero-beat, (equal to $\mathrm{LO}$ frequency), to $2 \mathrm{kHz}$ on the other side of twice the $\mathrm{LO}$, (twice because of the frequency doubler). Then for determining the opposite sideband suppression, the difference between the USB and LSB levels, in $\mathrm{dBm}$, was calculated as the opposite sideband suppression, or image rejection, in $\mathrm{dB}$.

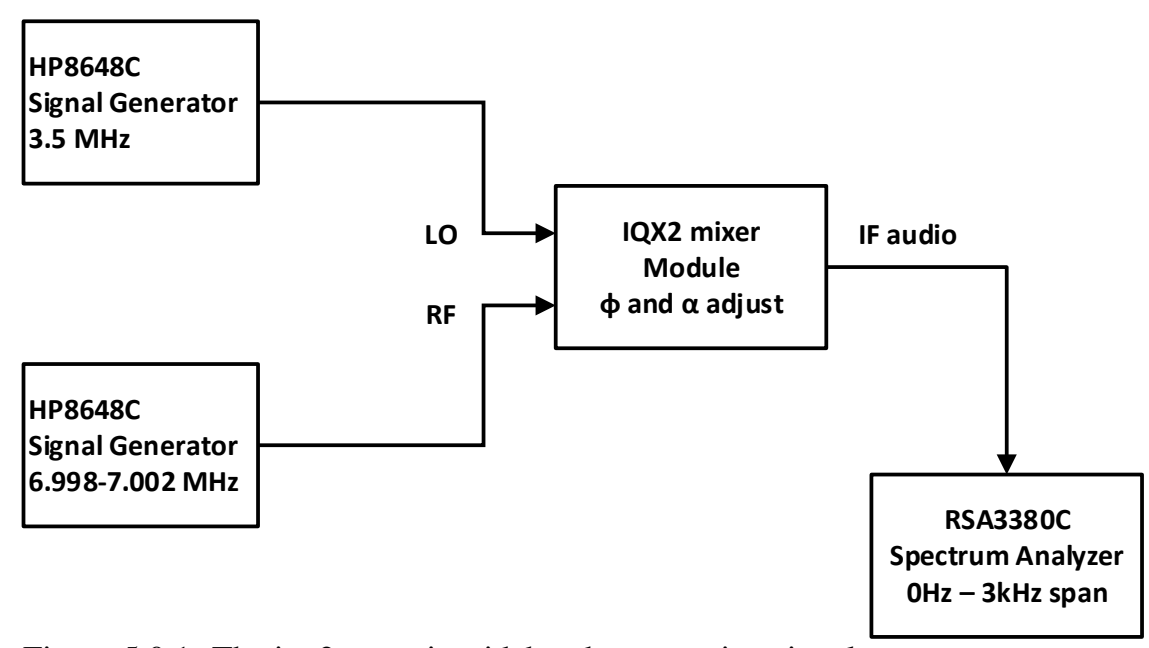

Figure 5.0.1: The iqx2 opposite sideband suppression signal generator test setup.

Measurements with signal generators, Figure 5.0.1, iqx2 showed that a drive-level of 


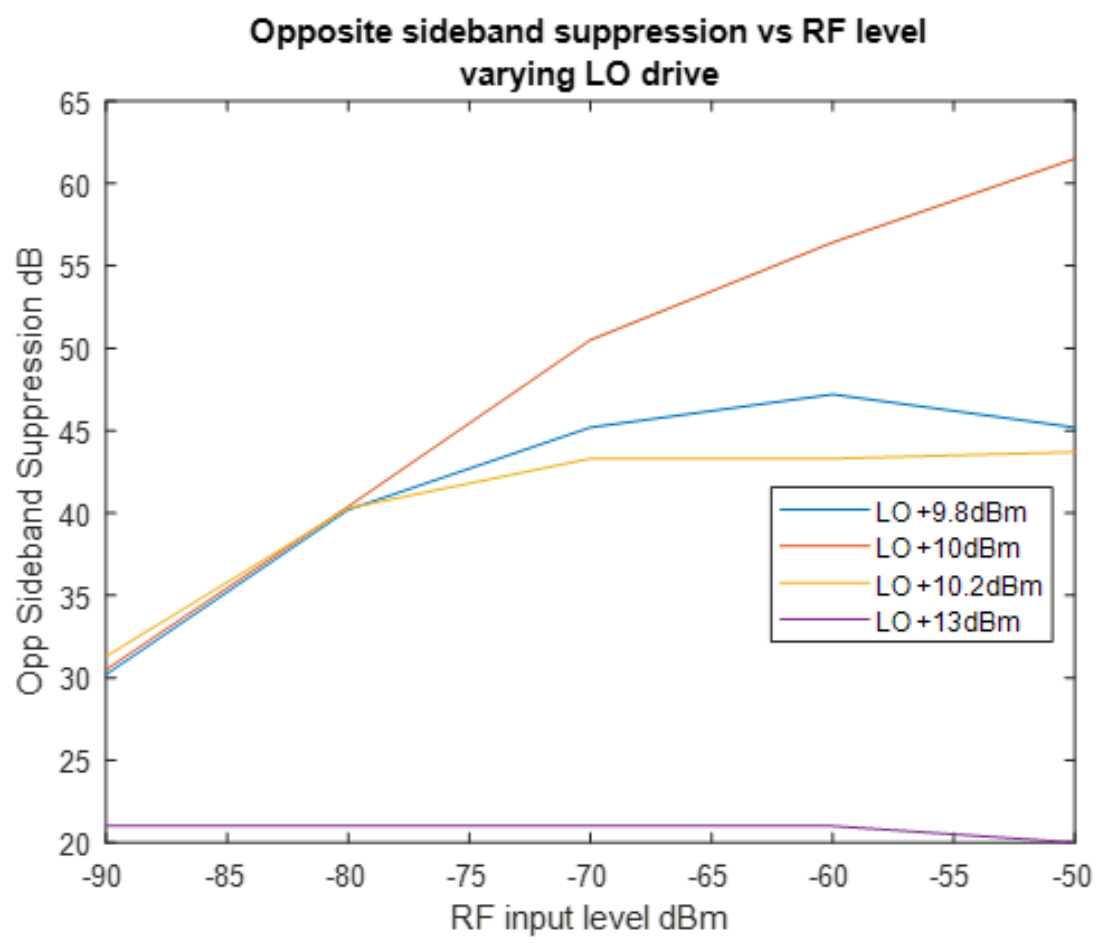

Figure 5.0.2: The iqx2 opposite sideband suppression sensitivity to LO drive-level with the signal generator test setup of Figure 5.0.1 at 3.5 MHz LO.

+10 dBm yielded the highest opposite sideband suppression, sweeping RF input levels from $-50 \mathrm{dBm}$ to $-90 \mathrm{dBm}$, Figure 5.0.2, and tuning the amplitude adjust in the iqx 2 . The LO frequency was fixed at $3.5 \mathrm{MHz}$ for this test. The opposite sideband suppression was then tested across the band, (for three LO frequencies), by varying the LO drive-level while keeping the RF input level fixed at $-60 \mathrm{dBm}$, Figure 5.0.3. It is not as clear from the LO drive-level sweep over frequency, Figure 5.0.3, that $+10 \mathrm{dBm}$ is the best choice. This is because $+10.6 \mathrm{dBm}$, at the top of the band, produced higher opposite sideband suppression, but $+10 \mathrm{dBm}$ held $45 \mathrm{~dB}+$ opposite sideband suppression across the band, so this was chosen as the optimal LO drive-level.

The first scaled VFO the author built, scaled from $7 \mathrm{MHz}$ to $3.5 \mathrm{MHz}$, resulted in lower output power-about $+8 \mathrm{dBm}$ versus $+10 \mathrm{dBm}$. The author tried to think of a convenient way to increase the output power level without changing the power supply and the board voltage regulator setup. The author observed that, in the scaling process, the coupling ca- 


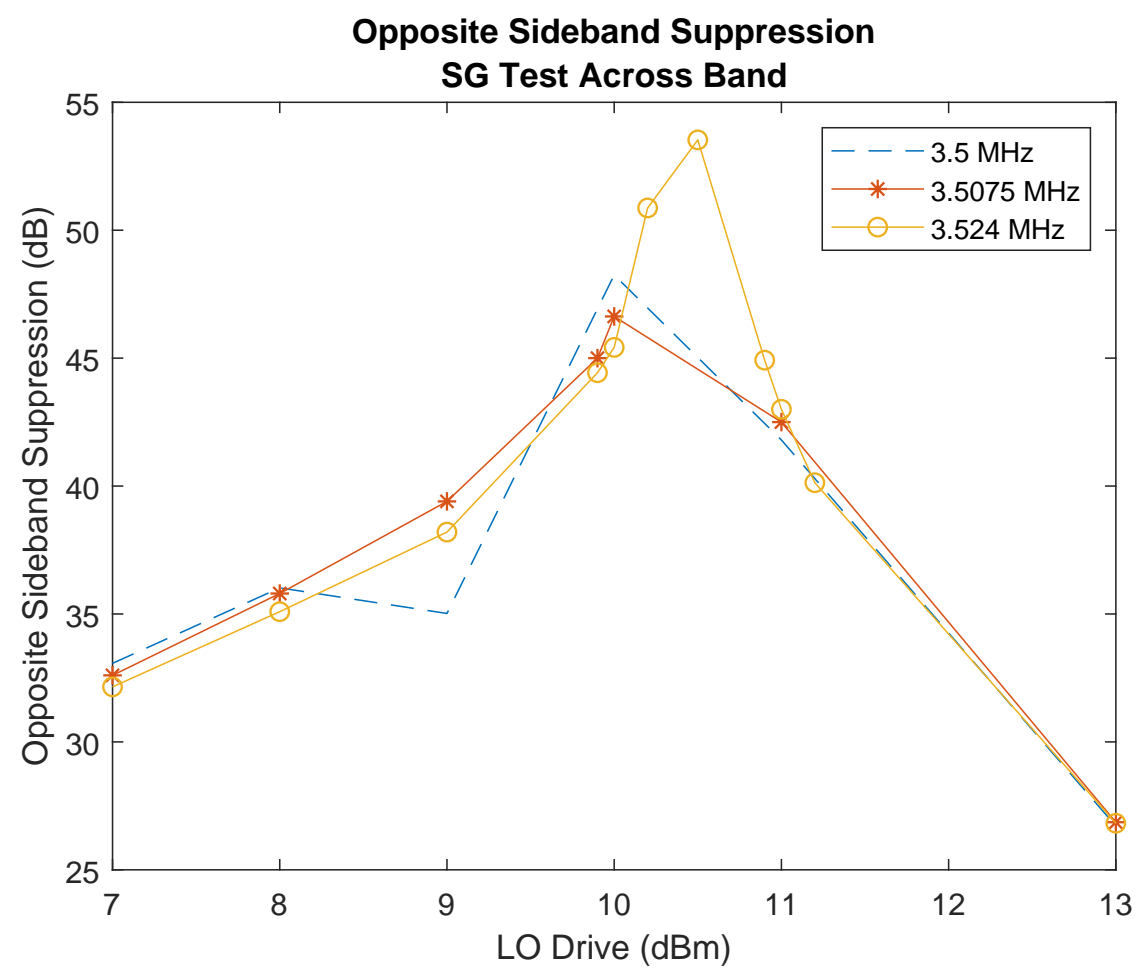

Figure 5.0.3: The iqx2 opposite sideband suppression sensitivity to LO drive-level and frequency with the signal generator test setup of Figure 5.0.1 RF input level fixed at $-60 \mathrm{dBm}$.

pacitor on the JFET gate had not been touched. The halving of the oscillator frequency meant that the impedance of this coupling capacitor was effectively doubled, which consequently increased the voltage drop across it. The author predicted that if the capacitance was scaled up by about 2 , then—being in series with the tank voltage — the voltage drop across the coupling capacitor would decrease, and the voltage swing across the tank would therefore increase. The output power increased to roughly $+10 \mathrm{dBm}$ when the coupling capacitor was increased to $5.8 \mathrm{pF}$ from the previous $2.9 \mathrm{pF}$, supporting the prediction. This was an experimental result, not rigorously informed from theory, but it produced the desired output power level and was used to replicate the signal generator test results. Later tests, discussed in this chapter, revealed the errors of this result, and the coupling capacitor value was accordingly decreased to the $4.7 \mathrm{pF}$ shown in Figure 4.0.3.

The problem was that $+10 \mathrm{dBm}$ from the VFO did not yield anywhere near the 45 $\mathrm{dB}+$ opposite sideband suppression results observed from the signal generator test, (Fig- 
Table 5.1: The iqx2 opposite sideband suppression with VFO utilizing variable coupler to vary LO drivelevel.

\begin{tabular}{|c|c|c|c|c|c|c|}
\hline $\begin{array}{c}\mathrm{LO} \\
(\mathrm{MHz})\end{array}$ & $\begin{array}{l}\text { x2 LO } \\
(\mathrm{MHz})\end{array}$ & $\begin{array}{c}\text { LO level } \\
(\mathrm{dBm})\end{array}$ & $\begin{array}{c}\text { RF level } \\
(\mathrm{dBm})\end{array}$ & $\begin{array}{c}\text { USB } \\
\text { (dBm) }\end{array}$ & $\begin{array}{c}\text { LSB } \\
(\mathrm{dBm})\end{array}$ & $\begin{array}{l}\text { OSS } \\
\text { (dB) }\end{array}$ \\
\hline \multirow{3}{*}{3.4826} & \multirow{3}{*}{6.9653} & 10.62 & -60 & -35.4 & -59.1 & 23.7 \\
\hline & & \multirow{2}{*}{10.6} & -50 & -26.35 & -48.19 & 21.84 \\
\hline & & & -50 & -26.4 & -48.8 & 22.4 \\
\hline 3.4739 & 6.9478 & 10.29 & -50 & -26.55 & -52.8 & 26.25 \\
\hline 3.4710 & 6.9420 & 10.16 & -50 & -26.59 & -53 & 26.41 \\
\hline \multirow{2}{*}{3.4691} & \multirow{2}{*}{6.9381} & \multirow{2}{*}{10} & -50 & -26.5 & -46.14 & 19.64 \\
\hline & & & -60 & -36.1 & -56.02 & 19.92 \\
\hline \multirow{5}{*}{3.4665} & \multirow{5}{*}{6.9329} & 9.9 & -50 & -26.7 & -56.5 & 29.8 \\
\hline & & \multirow{4}{*}{9.85} & -50 & -26.9 & -58.1 & 31.2 \\
\hline & & & -60 & -36.42 & -67.8 & 31.38 \\
\hline & & & -70 & -46.41 & -77.8 & 31.39 \\
\hline & & & -80 & -56.4 & -85.9 & 29.5 \\
\hline 3.4649 & 6.9297 & 9.87 & -50 & -26.58 & -57.5 & 30.92 \\
\hline
\end{tabular}

ures 5.0.2 and 5.0.3). Opposite sideband suppression levels of about $22 \mathrm{~dB}$ were observed with the VFO for LO level $+10 \mathrm{dBm}$, Table 5.1. Since the $+10 \mathrm{dBm}$ LO drive-level did not match the expected results, the drive-level output was varied to search for the most optimal LO level with the free-running VFO setup. At first, the output drive-level was varied by replacing the JFET gate coupling capacitor with a variable capacitor, informed from previous experimental results, which increased the output drive-level. This produced poor results, Table 5.1, where the best suppression was $31 \mathrm{~dB}$ at $+9.85 \mathrm{dBm}$ drive-level.

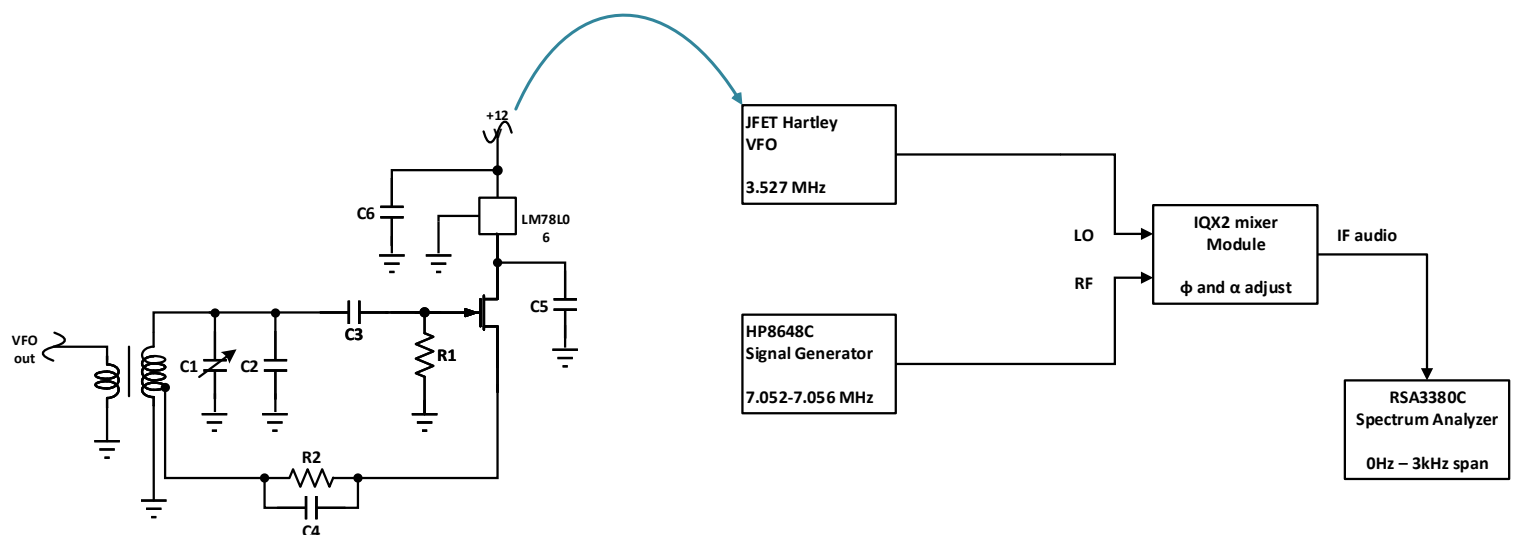

Figure 5.0.4: The iqx2 opposite sideband suppression test setup with signal generator for RF input and VFO for local oscillator (LO). 
The variable capacitor did indeed produce a variable output drive, but at a cost that the author had not understood; the output frequency was also varying based on the output drive-level. But it was varying by an amount that suggested that the JFET's gate-source capacitor $\left(C_{g s}\right)$ was participating in the LC tank. An unaccounted for $4 \mathrm{pF}$ or so would be affecting the output frequency at the highest output power-levels. The J310 JFET datasheet stated that the nominal $C_{g s}$ was about $4 \mathrm{pF}$, which corroborated the observed stray $4 \mathrm{pF}$ of frequency variation. Through private correspondence with Wes Hayward (W7ZOI) [17], and further reading from the oscillator section of EMRFD [4], the purpose of the coupling capacitor was understood. The gate coupling capacitor couples loop power back to the gate, but it also forms a capacitive divider with the JFET's $C_{g s}$. This can be seen in the equivalent small-signal model of Figure 5.0.5, where $C_{3}$ is the variable coupling capacitor. The larger the coupling capacitor-relative to the JFET's $C_{g s}$ - the larger the oscillator's output power, and the more the $C_{g s}$ capacitor participated in the LC tank oscillation frequency. The coupling capacitor is therefore recommended to be as small as possible for reliable starting [22], to ensure isolation between $C_{g s}$ and the tank.

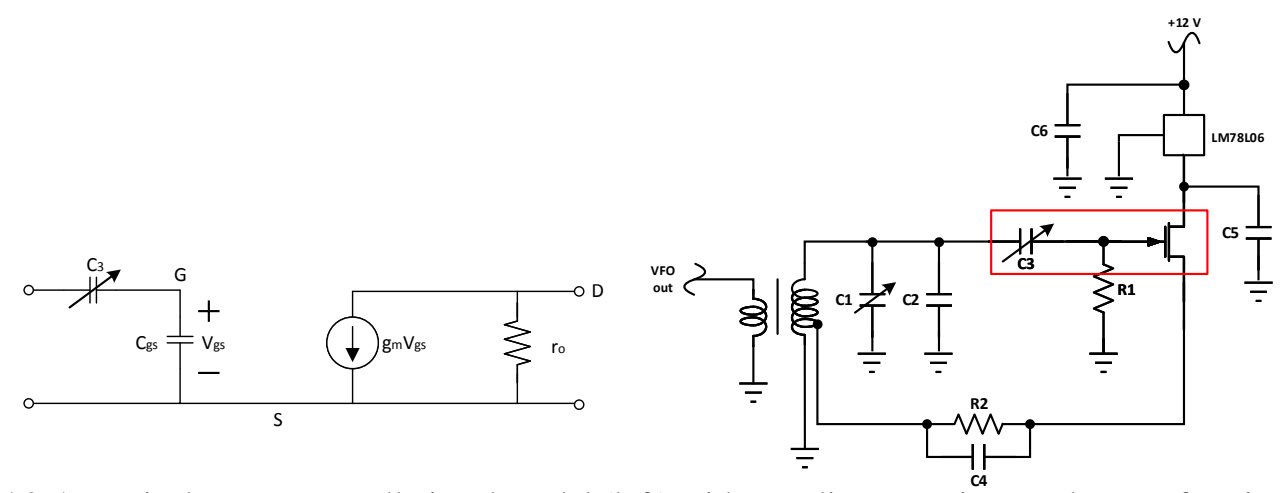

Figure 5.0.5: Equivalent JFET small-signal model (left) with coupling capacitor on the gate forming voltage divider with $C_{g s}$.

As the $+10 \mathrm{dBm}$ LO drive did not serve to replicate the signal generator test results, and tuning the output power of the VFO with the variable coupling capacitor on the gate did not serve to produce anything much better, the author turned to other potential culprits. The 
author thought that this might be due to the VFO possessing poor phase noise, but review of the literature of this Hartley oscillator [22] suggested this was the wrong conclusion. The expected phase noise from previously measured versions of this circuit were about $-146 \mathrm{dBc} / \mathrm{Hz}$ at $20 \mathrm{kHz}$ offset [4] [22]. The author attempted to measure the phase noise in the lab with available equipment, but encountered instrument limitations. The signal generators used for the tests (HP8648C) had phase noise, stated in the datasheet, to be $<-$ $120 \mathrm{dBc} / \mathrm{Hz}$ at a $500 \mathrm{MHz}$ carrier [41]. The datasheet did not have data for 3.5 MHz center frequency, but it may be expected to be a little better than $-120 \mathrm{dBc} / \mathrm{Hz}$.

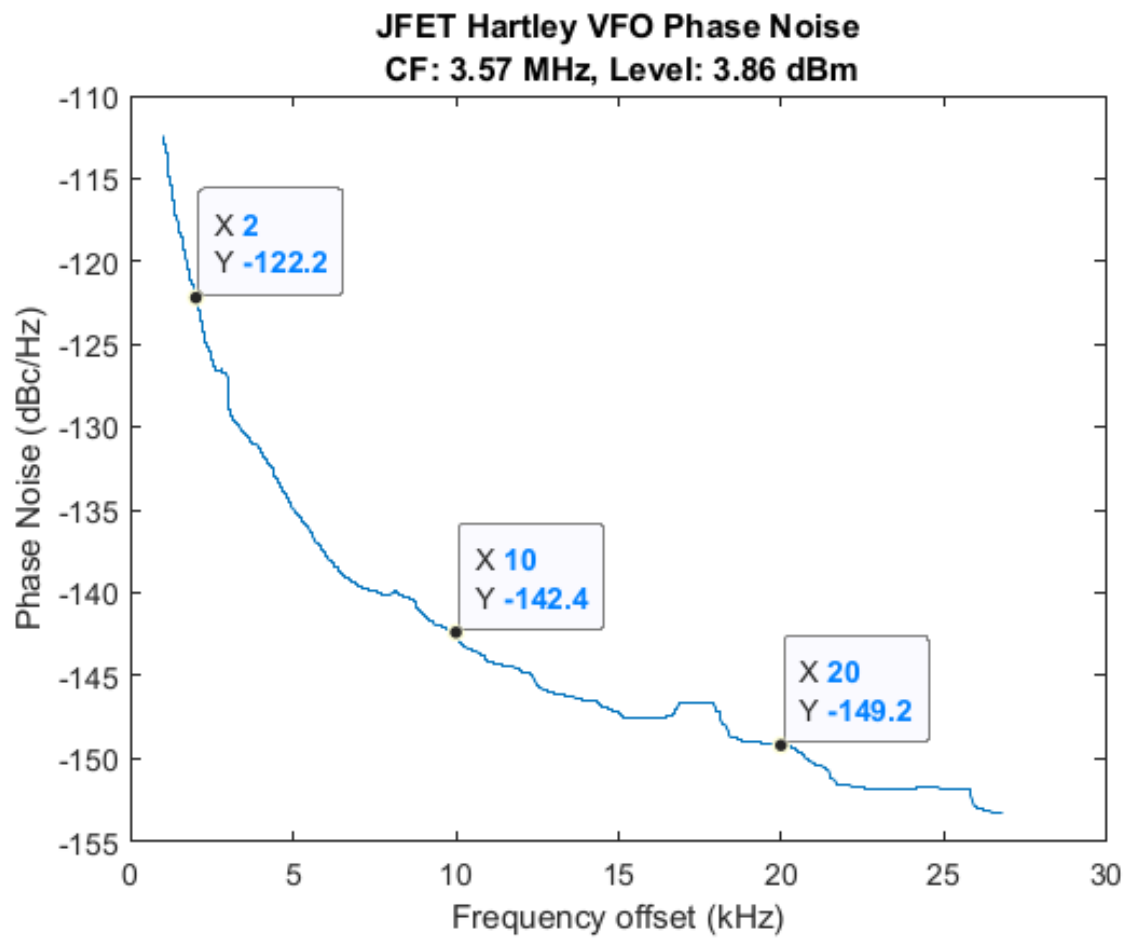

Figure 5.0.6: Phase noise of VFO measured with R\&S FSWP.

The signal generator phase noise could not be measured at the $20 \mathrm{kHz}$ offset, however, with the spectrum analyzer the author had at hand (RSA3308C), because its own phase noise — at a $20 \mathrm{kHz}$ offset from a $1 \mathrm{GHz}$ carrier-was - $108 \mathrm{dBc} / \mathrm{Hz}$ [42]. Thus the spectrum analyzer's own internal phase noise was likely to be worse than the DUT's as well as the signal generator's, meaning too much phase noise to reliably measure the DUT's own phase 
noise. The author was able to use a Rohde \& Schwarz phase noise spectrum analyzer (FSWP) at work, and measured $-149 \mathrm{dBc} / \mathrm{Hz}$ at a $20 \mathrm{kHz}$ offset, Figure 5.0.6. The phase noise measurements were made with the VFO unshielded and open in the lab.

After the phase noise measurements, the author was convinced that the phase noise of the oscillator was not leading to the poor opposite sideband suppression. Instead, the author investigated other fundamental differences between the two setups: signal generator for LO, vs. free-running Hartley VFO for LO. One significant difference was that the signal generator provided a wideband $50 \Omega$ load to the frequency doubler input, and the VFO output was not $50 \Omega$ as it was a 3-turn link directly off the JFET Hartley tank inductor. Therefore a $3 \mathrm{~dB}$ minicircuits attenuator was acquired, as it was lying around the lab, and placed in-between the VFO and the iqx2 LO input to the frequency doubler, Figure 5.0.7.

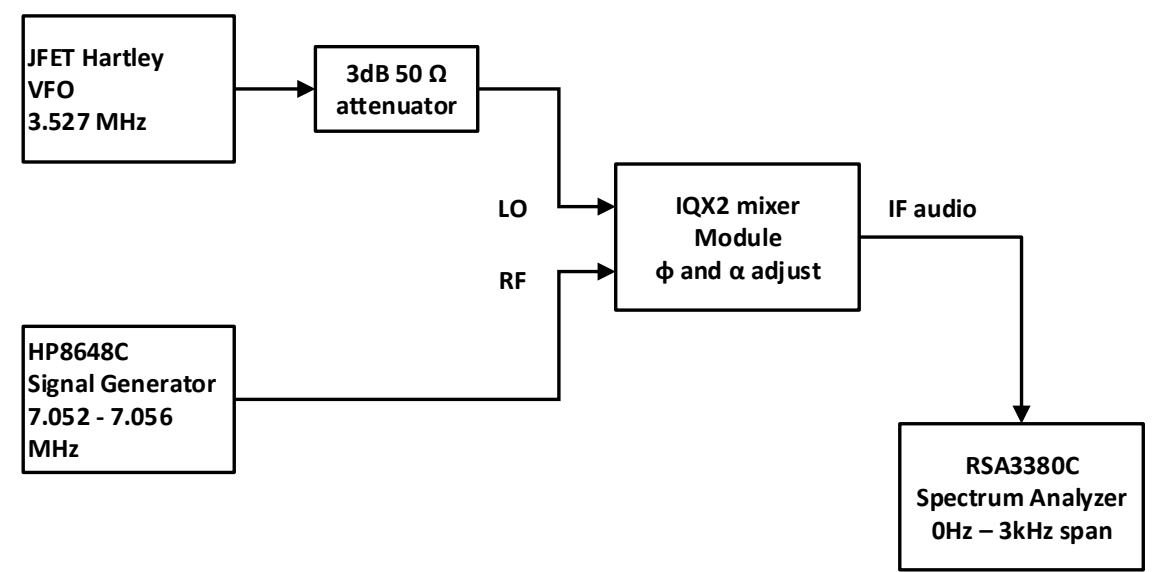

Figure 5.0.7: The iqx2 opposite sideband suppression signal generator test setup with $3 \mathrm{~dB}$ attenuator to provide $50 \Omega$ input between VFO and the iqx2 input.

This immediately produced nearly the results expected from the signal generator test; with the difference that the iqx2 now required less LO drive than the signal generator test predicted. It was now producing up to $60 \mathrm{~dB}$ of opposite sideband suppression, (nonuniformly across the band), with $+3.7 \mathrm{dBm}$ LO drive-measured after the attenuator. This was about $6 \mathrm{~dB}$ less than the signal generator setup required. The $3 \mathrm{~dB}$ attenuator forced the VFO output impedance presented to the frequency doubler to be a lot closer to $50 \Omega$ 
and brought the two test setups closer to a valid comparison. This test setup, with the $3 \mathrm{~dB}$ attenuator, can be seen in Figure 5.0.7. The final opposite sideband suppression with the 3 $\mathrm{dB}$ attenuator can be seen in Table 5.2. Above $40 \mathrm{~dB}$ opposite sideband suppression was achieved for small variation in power supply $(4.2-4.5 \mathrm{~V})$ and LO drive-levels $+2.25 \mathrm{dBm}$ $-+5 \mathrm{dBm}$.

Table 5.2: The iqx2 opposite sideband suppression with temperature compensated VFO utilizing the test setup of Figure 5.0.7. 4.7pF non-NP0 ceramic capacitor used for coupler on JFET gate.

\begin{tabular}{|c|c|c|c|c|c|c|c|c|}
\hline $\begin{array}{c}\text { current iqx2 } \\
\mathrm{mA}\end{array}$ & $\begin{array}{c}\text { voltage } \\
\text { V }\end{array}$ & $\begin{array}{c}\text { LO level } \\
\text { dBm }\end{array}$ & $\begin{array}{c}\text { LO Freq } \\
\mathrm{MHz}\end{array}$ & $\begin{array}{c}\text { RF level } \\
\mathrm{dBm}\end{array}$ & $\begin{array}{l}\text { USB } \\
\mathrm{dBm}\end{array}$ & $\begin{array}{c}\text { LSB } \\
\mathrm{dBm}\end{array}$ & $\begin{array}{l}\text { OSS } \\
\mathrm{dB}\end{array}$ & $\begin{array}{c}\text { x2 LO Freq SB } \\
\text { MHz (USB) }\end{array}$ \\
\hline \multirow{2}{*}{8} & \multirow{2}{*}{7} & \multirow{2}{*}{10.05} & \multirow{2}{*}{3.4883} & -50 & -27.35 & -49.8 & 22.45 & \multirow{2}{*}{6.9787} \\
\hline & & & & -60 & -37.04 & -59.8 & 22.76 & \\
\hline 8 & 6.98 & 10.01 & 3.4883 & -60 & -37.08 & -59.98 & 22.9 & 6.9787 \\
\hline \multirow{3}{*}{6} & \multirow{3}{*}{5} & \multirow{3}{*}{6.6} & \multirow{3}{*}{3.4898} & -60 & -38.57 & -70.5 & 31.93 & \multirow{3}{*}{6.9816} \\
\hline & & & & -50 & -28.96 & -60.66 & 31.7 & \\
\hline & & & & -70 & -48.9 & -80.56 & 31.66 & \\
\hline \multirow{2}{*}{6} & \multirow{2}{*}{4.5} & \multirow{2}{*}{5.04} & \multirow[b]{2}{*}{3.49041} & -60 & -38.88 & -80.3 & 41.42 & \multirow{2}{*}{6.9828} \\
\hline & & & & -50 & -30.3 & -70.7 & 40.4 & \\
\hline \multirow{2}{*}{6} & \multirow{2}{*}{4.4} & \multirow{2}{*}{4.54} & \multirow[b]{2}{*}{3.490545} & -60 & -40.34 & -85.6 & 45.26 & \multirow{2}{*}{6.9831} \\
\hline & & & & -50 & -30.52 & -77.2 & 46.68 & \\
\hline \multirow{2}{*}{6} & \multirow{2}{*}{4.39} & \multirow{2}{*}{4.47} & \multirow[b]{2}{*}{3.49055} & -60 & -40.41 & -82.8 & 42.39 & \multirow{2}{*}{6.9831} \\
\hline & & & & -50 & -30.58 & -73.5 & 42.92 & \\
\hline \multirow{2}{*}{6} & \multirow{2}{*}{4.3} & \multirow{2}{*}{3.74} & \multirow[b]{2}{*}{3.490675} & -50 & -31.01 & -91 & 59.99 & \multirow{2}{*}{6.9834} \\
\hline & & & & -40 & -22.48 & -79.2 & 56.72 & \\
\hline \multirow{3}{*}{6} & \multirow{3}{*}{4.25} & \multirow{3}{*}{2.76} & & -50 & -31.3 & -84.5 & 53.2 & \multirow{3}{*}{6.9835} \\
\hline & & & & -60 & -41.16 & -92 & 50.84 & \\
\hline & & & 3.490735 & -70 & -51.16 & -93 & 41.84 & \\
\hline 6 & (1) & 25 & & -50 & -31.55 & -81.2 & 49.65 & 60836 \\
\hline 0 & 4.2 & 2.20 & 3.490795 & -60 & -41.42 & -90 & 48.58 & 0.9030 \\
\hline 6 & 4 & 056 & & -60 & -43.3 & -79.7 & 36.4 & 69840 \\
\hline 0 & 4 & 0.50 & 3.49098 & -50 & -33.4 & -69.7 & 36.3 & \\
\hline
\end{tabular}




\section{Chapter 6 VFO Frequency Drift}

Once the driftiness of the VFO was observed and communicated to the author's professor, he suggested that the source of the driftiness - considering the component choices—was from the tank components. He subsequently supplied the author with higher quality parts. Immediately upon replacing the poly trimmer capacitor and ordinary ceramic capacitors with an air-core sliding-plate variable capacitor and silver mica capacitors, the oscillator output could be seen on the spectrum analyzer without whizzing off the screen. This was enough to prove that the temperature variability was arising from the temperature variability of the tank components, so at a minimum the tank capacitors were implicated.

A search for frequency stabilizing methods, including a method by Wes Hayward [14], was conducted in this work with the advice of the author's professor. Wes Hayward's method was attractive in the sense that the needed test equipment was at hand. Construction of additional equipment was easily achieved and explained in the article. The list of required equipment for the temperature compensation tests and the equipment used is as follows:

1. Frequency Counter : CMC251

2. Thermometer : partial-immersion (red spirit)

3. Temperature Chamber : styrofoam cooler

4. Heat source : light bulb

5. Separator : piece of bamboo board

6. Fan : 12v computer GPU fan (optional)

The author built the recommended temperature chamber from reference [14]. The chamber can be seen in Figure 6.0.1. The left picture is a closed-view of the chamber, with the styrofoam box (3), the thermometer sticking out (2), the VFO power supply and output cables below to the left, and the fan (6) with a removable cover below on the ground. 

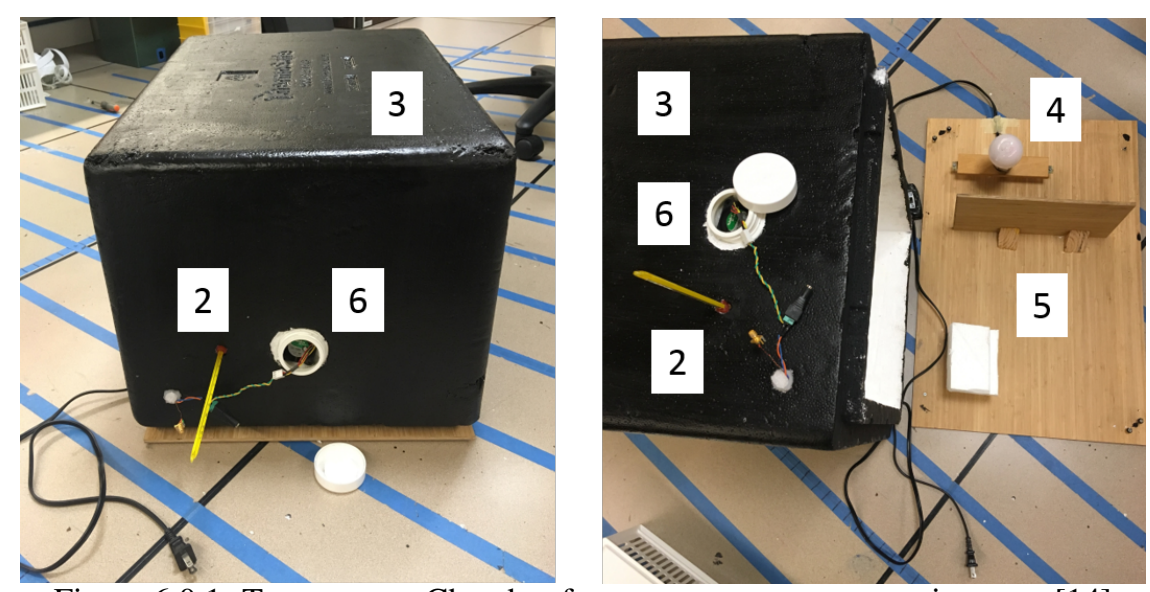

Figure 6.0.1: Temperature Chamber for temperature compensation tests [14]

The thermometer could alternatively be a digital one, but calibration would be needed [14]. The fan was installed into the side of the chamber to fascilitate removing heat from inside the chamber. An additional fan could be used internally to help improve thermal conduction, but care would have to be expended so that mechanical stress is not imparted to the DUT, [14].

The right picture of figure 6.0.1 is an open-view showing the light-bulb heat-source (4), separator (5) for the DUT, and a small platform for the DUT in the bottom left corner. The separator is a miniature internal wall that is needed to keep the light-bulb radiation from directly impacting the DUT. The platform was used to elevate the DUT because heat tends to rise, and the thermometer was placed at the same level as the DUT. A piece of bamboo board was used both for the separator and for the base of the temperature chamber. Styrofoam works very well as it is a good insulator. Only 3 sides and the top of the chamber were styrofoam, but the thermal insulation was still sufficient.

The basic idea of the temperature compensation technique was to measure the temperature variability of the oscillator and, with some basic assumptions, calculate the temperature coefficients (TC) of the tank components and replace some tank components with parts possessing equal and opposite temperature coefficients. 


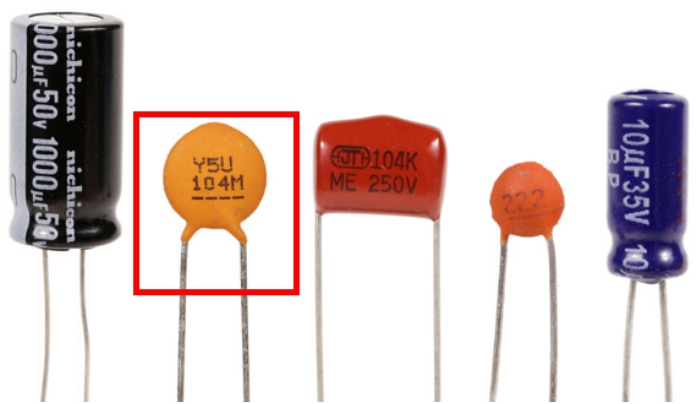

Figure 6.0.2: On the two ends are electrolytic capacitors, in the center is a film capacitor, and the remaining two are class 2 ceramic capacitors.

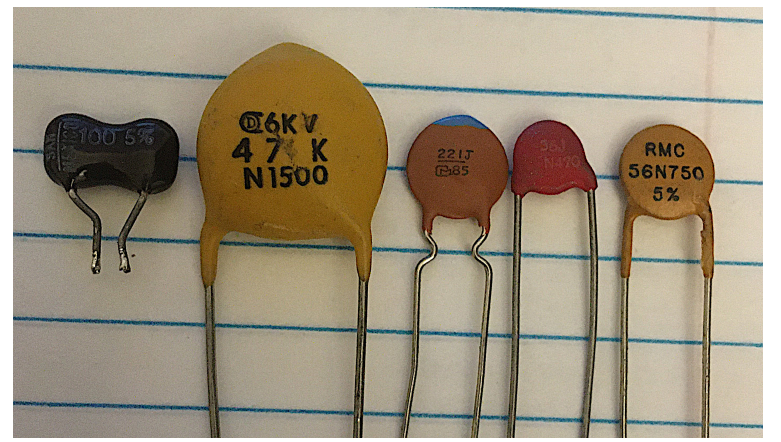

Figure 6.0.3: First is a silver mica capacitor, the following 4 are class 1 ceramic capacitors of different temperature coefficients.

$$
T C F=\frac{\Delta f}{f}=-\frac{1}{2}\left[T C L+T C_{C 1} \frac{C_{1}}{C_{t o t}}+T C_{C 2} \frac{C_{2}}{C_{t o t}}+\ldots\right]
$$

First the assumptions are important, the previously overlooked "NP0" and "COG" markers on schematics and components now had a solid and valid meaning to the author. The meaning of these codes is that a component, usually capacitor, marked with these codes"NP0" or "COG"- has a zero temperature coefficient. This means it will not change its values with temperature changes, with some tolerance-roughly $+/-30 \mathrm{ppm} /{ }^{\circ} \mathrm{C}$. There are two classes of ceramic capacitors. Class 1 have a well-defined temperature coefficient in partsper-million (ppm) per degree Celcius temperature change. Class 2 on the other hand are specified as a maximum percentage change over their entire operating temperature range for any deviation from room temperature, $\left(25^{\circ} \mathrm{C}\right)$. For example, the the rightmost class 1 capacitor in Figure 6.0.3 has a temperature coefficient of $-750 \mathrm{ppm} /{ }^{\circ} \mathrm{C}$ over its operating 


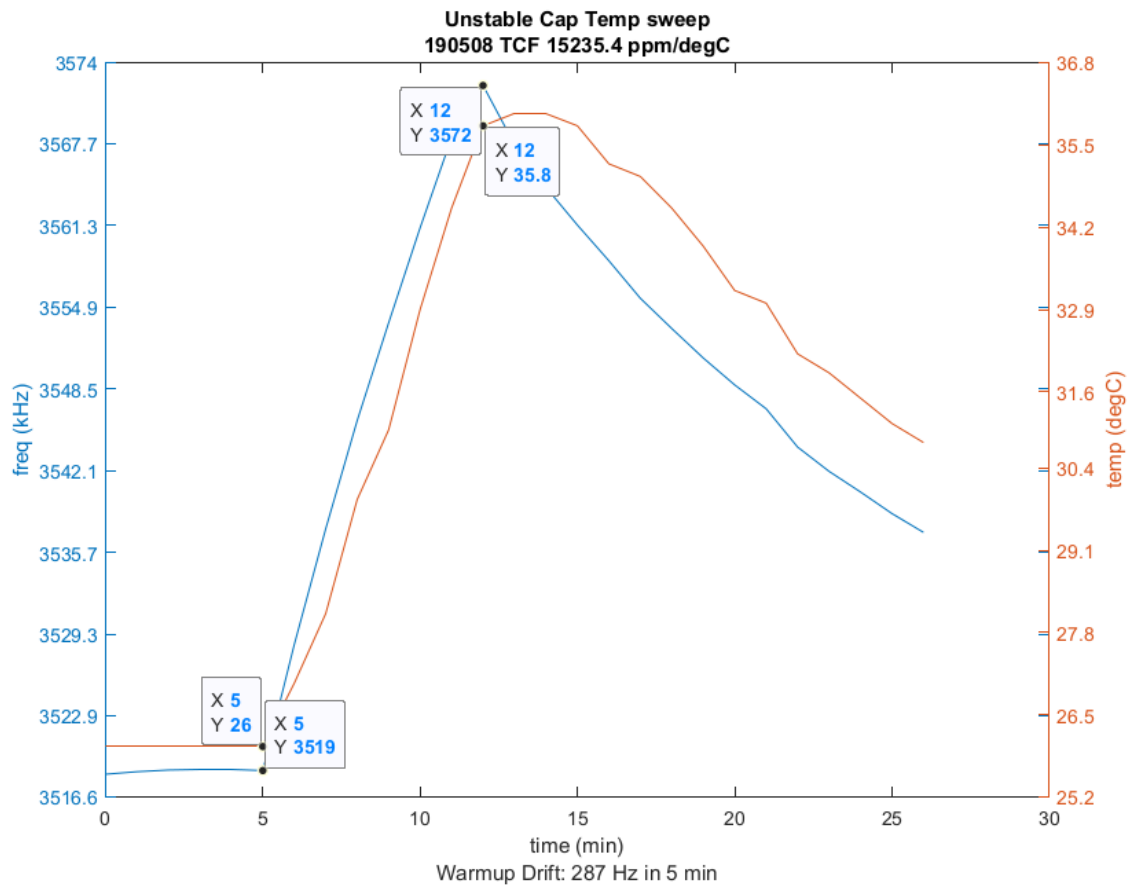

Figure 6.0.4: VFO drift with initial parts available from junk box.

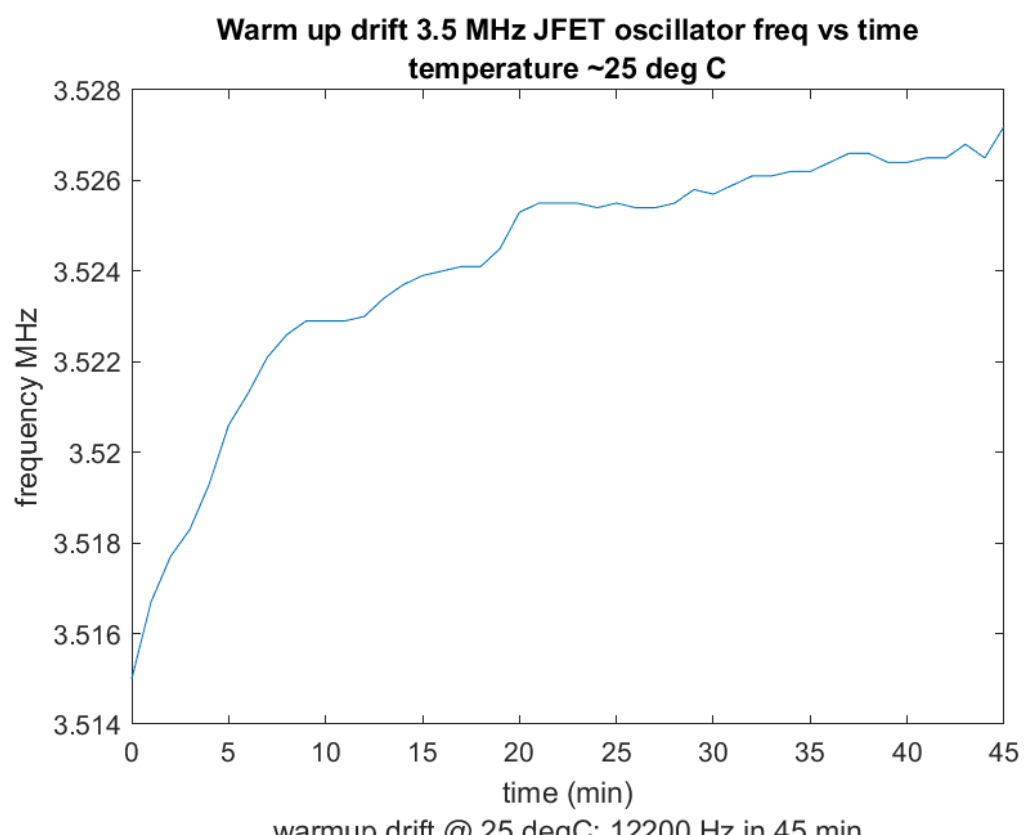

Figure 6.0.5: VFO warmup drift with initial parts available from junk box, $12.2 \mathrm{kHz}$ in $45 \mathrm{~min}$ and climbing! 
temperature range, with $5 \%$ value tolerance. In contrast, the boxed capacitor in Figure 6.0.2 is a class 2 ceramic capacitor with maximum percent capacitance change $+/-22 \%$ over its temperature range, $\left(-30{ }^{\circ} \mathrm{C}\right.$ to $\left.+85^{\circ} \mathrm{C}\right)$, with $20 \%$ value tolerance. This corresponds to about $220,000 \mathrm{ppm}$ maximum variation for an unspecified degree deviation from room temperature. Class 2 ceramic capacitors are less stable with temperature variations than class 1 , but they are able to have much higher capacitance values for their size [47].

The idea is to replace all tank capacitors with stable $0 \mathrm{ppm} /{ }^{\circ} \mathrm{C}$ parts and to measure the temperature coefficient of frequency (TCF) in a controlled temperature environment. The time, temperature, and the frequency are measured and recorded. The tank capacitors could be NP0/COG parts or silver mica capacitors, which also typically have low temperature coefficients. The tank inductor, being wound with magnetic wire on powdered iron toroid core, typically has some appreciable amount of positive temperature coefficient.

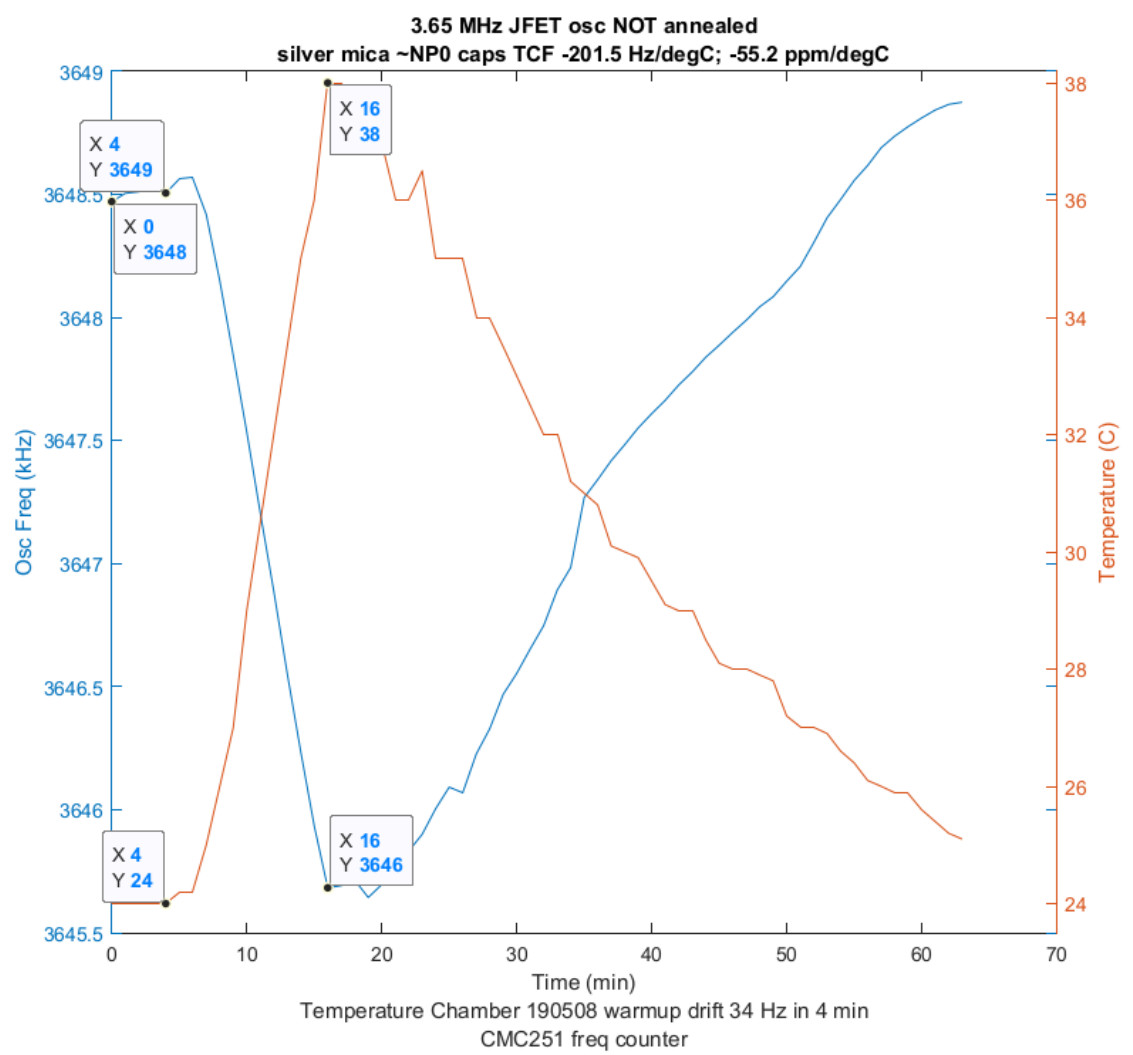

Figure 6.0.6: VFO drift after changing a few key components in the tank circuit. 
By replacing all the capacitors with zero temperature coefficient parts, the measured temperature drift can be assumed to be produced solely by the inductor. Equation 6.0.1 [4] [14] gives the relationship between TCF and the inductor temperature coefficient (TCL) and temperature coefficients of the tank capacitors $\left(T C_{C i}\right)$. With the temperature coefficients of the capacitors all assumed to be zero, Equation 6.0.1 reduces to the measured TCF equaling half of the temperature coefficient of the inductor (TCL).

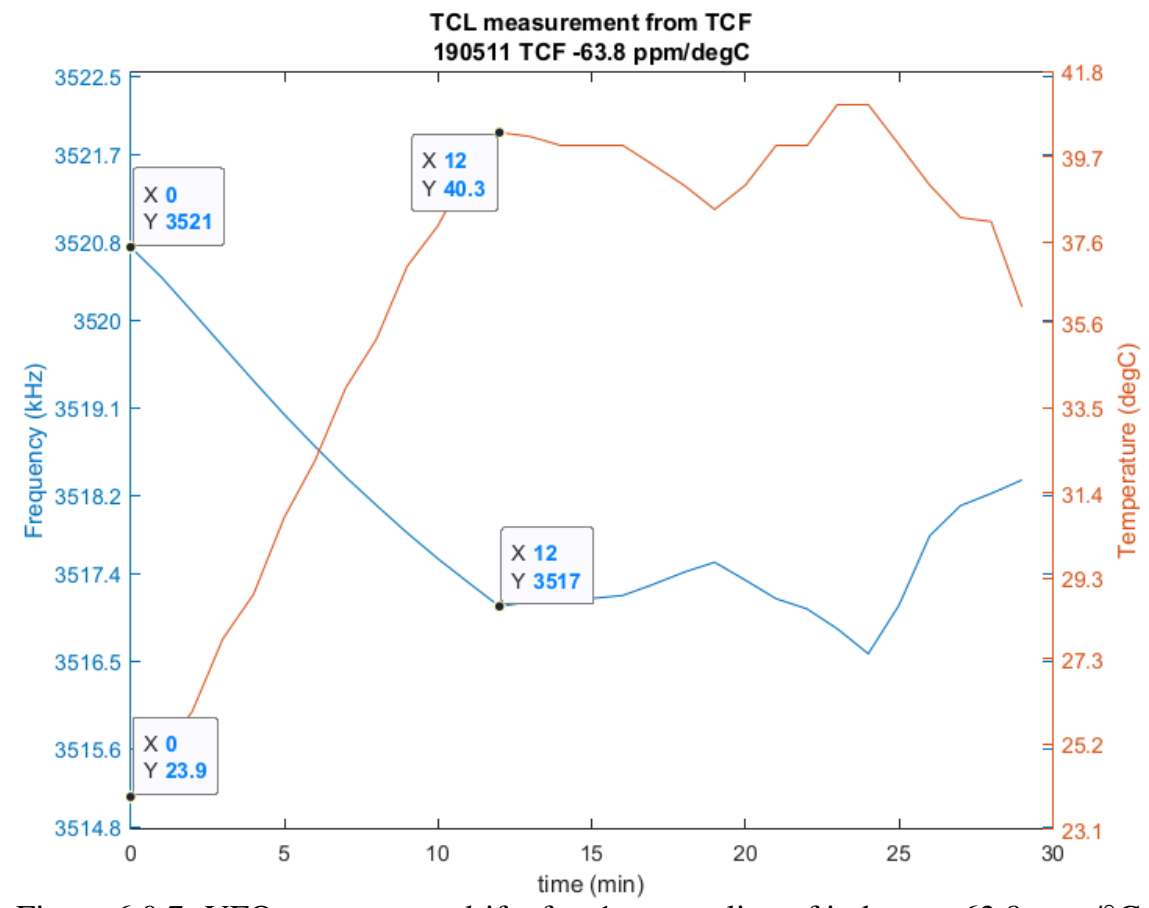

Figure 6.0.7: VFO temperature drift after $1 \mathrm{st}$ annealing of inductor $-63.8 \mathrm{ppm} /{ }^{\circ} \mathrm{C}$.

Before proceeding with the measurement of the TCF, the unstable junk box capacitors were swapped back in for the silver mica (SM) capacitors and air core variable capacitor. Figure 6.0.4 shows the original drift was about $+15235 \mathrm{ppm} /{ }^{\circ} \mathrm{C}$, or about $+11.5 \mathrm{kHz} /{ }^{\circ} \mathrm{C}$, so it is no wonder that this was visible on the spectrum analyzer. The warmup characteristic of the drifty circuit does not settle out even after 45 minutes, Figure 6.0.5; drifting about $12.2 \mathrm{kHz}$ and climbing.

The author next removed the original drifty components, replaced them with Dr. Campbell's stable silver mica and air core variable capacitor, and the temperature drift measured. 
Table 6.1: The tank components for calculation of the TCL of the inductor, and for measurements of Figures 6.0.7, 6.0.8, and 6.0.10

\begin{tabular}{|c|c|c|c|}
\hline QTY & Cap fixed & Cvar & Inductor \\
\hline 1 & $100 \mathrm{p}$ SM & $1.6 \mathrm{p}-11.2 \mathrm{p}$ & $11.3 \mu$ \\
\hline 1 & $56 \mathrm{p} \mathrm{SM}$ & & \\
\hline 1 & $10 \mathrm{p} \mathrm{NP0}$ & & \\
\hline
\end{tabular}

Figure 6.0.6 shows the result. A significant improvement in TCF can be seen in Figure 6.0.4, going from $+15235 \mathrm{ppm} /{ }^{\circ} \mathrm{C}$ to $-55 \mathrm{ppm} /{ }^{\circ} \mathrm{C}$. The temperature stable capacitor values could only achieve $3.65 \mathrm{MHz}$, but the results were instructive regardless-exposing the drifty quality of the original capacitors. More silver mica and NP0 components were acquired and the VFO tuned down to $3.5 \mathrm{MHz}$.

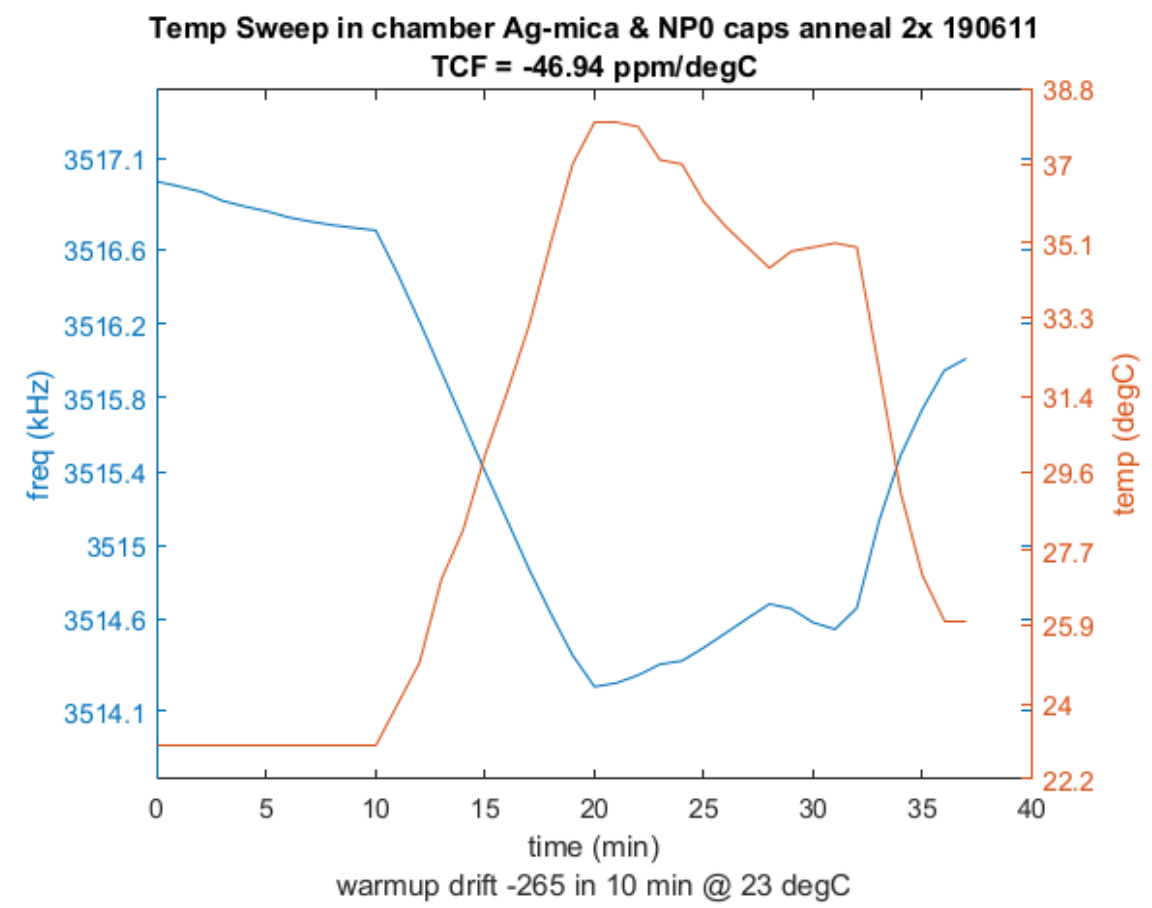

Figure 6.0.8: VFO temperature drift after 2nd annealing of inductor $-46.94 \mathrm{ppm} /{ }^{\circ} \mathrm{C}$.

A recommendation found in Roy Lewallen's paper [28] suggested that annealing the inductor, to temperature condition it, makes it less variable with temperature. The tapped inductor of the Hartley oscillator was wound on a powdered-iron toroid core with magnetically- 


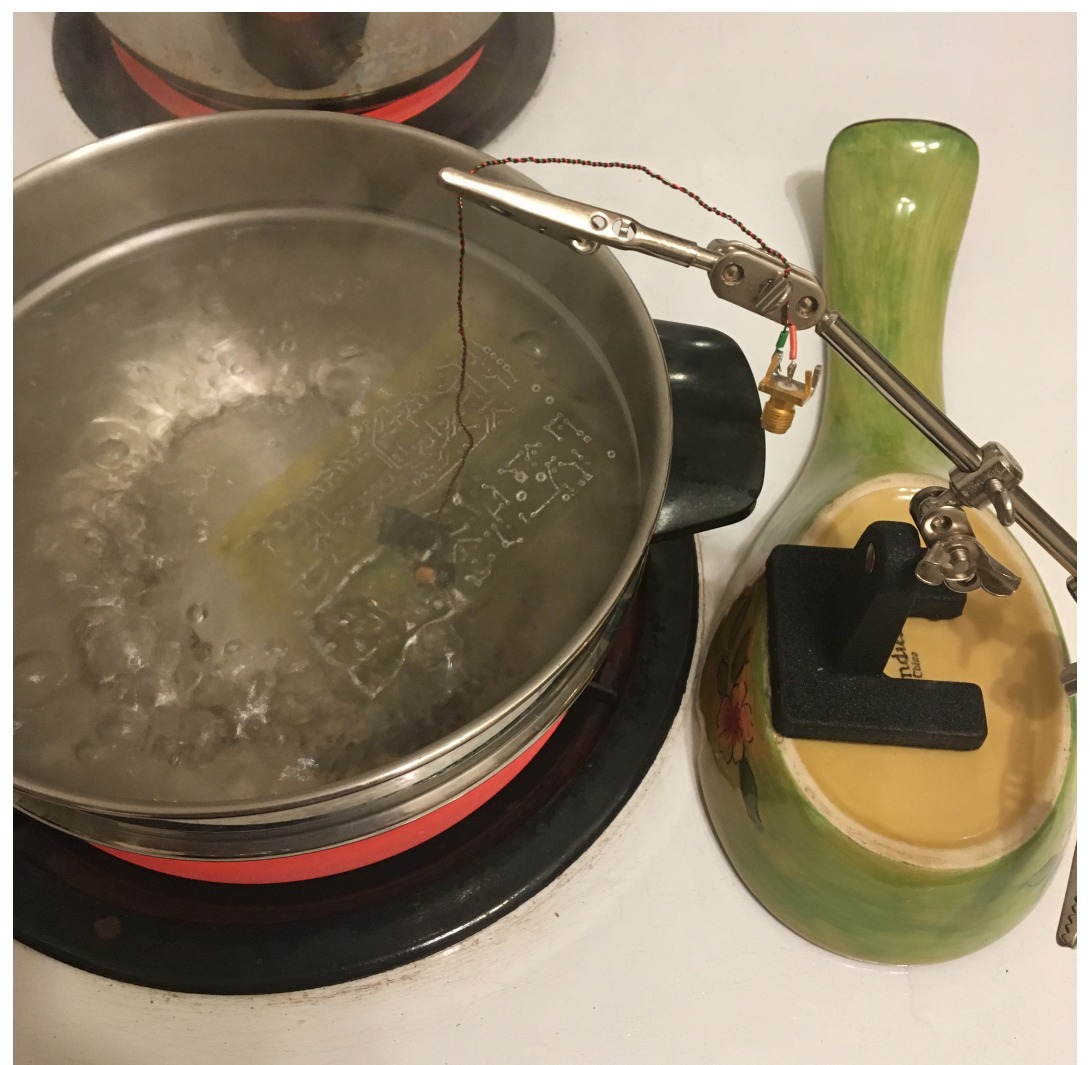

Figure 6.0.9: Boiling of entire circuit in pot of water on the stove to anneal inductor.

insulated wire and annealing likely temperature-conditions the wire windings to have a stable temperature coefficient. The resultant temperature drift of the $3.5 \mathrm{MHz}$ circuit after annealing one and two times can be seen in Figures 6.0.7 and 6.0.8, respectively. Figure 6.0.7 has $-63.8 \mathrm{ppm} /{ }^{\circ} \mathrm{C} \mathrm{TCF}$, and the 2 nd annealing of Figure 6.0 .8 shows marginal improvement to $-46.94 \mathrm{ppm} /{ }^{\circ} \mathrm{C} \mathrm{TCF}$. The warmup characteristics, however, became noticeably more stable with each annealing, Figure 6.0.10. The warmup drift before annealing is greater than $650 \mathrm{~Hz}$ in more than 70 minutes, with $500 \mathrm{~Hz}$ of the drift occurring in the first 20 minutes. After the first annealing the warmup improves to about $320 \mathrm{~Hz}$, most of which occurs in the first 17 minutes, but still continues to drift after 120 minutes. After the 2nd annealing, the warmup drift was about $100 \mathrm{~Hz}$, stabilizing well after about 17 minutes. The inductor had already been placed on the board, so the entire circuit was boiled in water for about 5 minutes for the first annealing, and 10 minutes for the second annealing. Figure 6.0.9 
shows the oscillator board in a pot of boiling water on the stove.

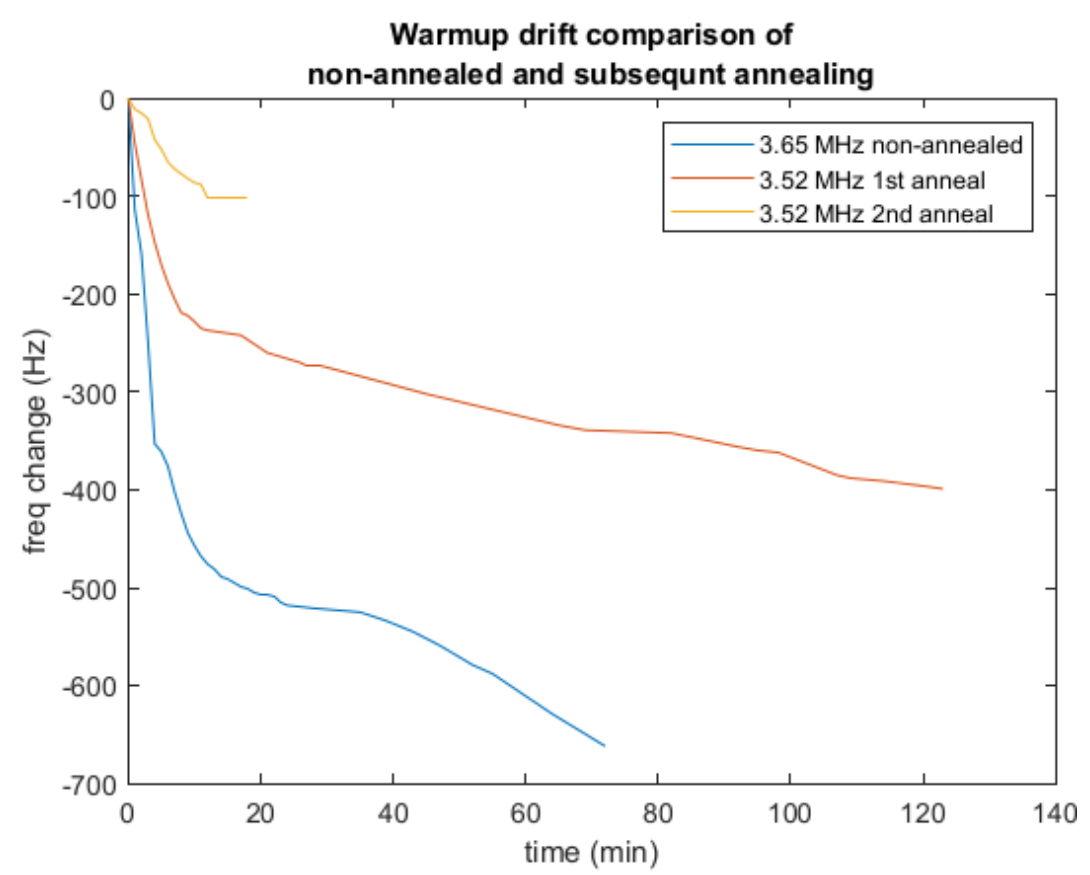

Figure 6.0.10: Comparison of warmup of oscillator before and after annealing one and two times.

$$
\begin{aligned}
T C L & =(-2) T C F-T C_{C 1} \frac{C_{1}}{C_{t o t}}-\left.T C_{C 2} \frac{C_{2}}{C_{t o t}}\right|_{T C_{c i}==0} \\
& =(-2)(-63.8) \\
& =+128 \frac{p p m}{{ }^{\circ} C} \\
T C L & =(-2) T C F-T C_{C 1} \frac{C_{1}}{C_{t o t}}-T C_{C 2} \frac{C_{2}}{C_{t o t}} \\
& =(-2)(-63.8)-(-50) \frac{100 p F}{177.2 p F}-(-50) \frac{56 p F}{177.2 p F} \\
& =+171 \frac{p p m}{{ }^{\circ} C}
\end{aligned}
$$

After the first annealing, the temperature coefficient of inductance (TCL) was calculated by rearranging Equation 6.0.1, to solve for the TCL, Equations 6.0.2 and 6.0.3. Equation 6.0.2 assumes ideal zero temperature coefficient capacitors, and thus the TCL is just $2 \mathrm{x}$ the measured TCF. Equation 6.0.3 introduces some component non-idealities, discussed in the 
following paragraphs.

$$
\begin{aligned}
\left.T C F\right|_{=0} & =-\frac{1}{2}\left[T C L+T C_{C_{1}} \frac{C_{1}}{C_{t o t}}\right] \\
T C_{C_{1}} & =-128\left(\frac{177.2 p}{56 p}\right) \\
& =-405 \frac{p p m}{{ }^{\circ} C}
\end{aligned}
$$

The author chose a $56 \mathrm{pF}$ capacitor as the compensating capacitor, and the temperature coefficient $\left(T C_{C 1}\right)$ was calculated using the ideal TCL of $+128 \mathrm{ppm} /{ }^{\circ} \mathrm{C}$ and a TCF of zero, Equation 6.0.4. The TC was calculated to be $-405 \mathrm{ppm} /{ }^{\circ} \mathrm{C}$, but the closest standard $\mathrm{TCs}$ were $-330(\mathrm{~N} 330)$ or $-470(\mathrm{~N} 470)$. Neither N330, nor N470 for $56 \mathrm{pF}$ could be located online or elsewhere. Instead, a heuristic measurement with an acquired 56 pF N750 capacitor was performed to test how well the mathematical model fit measurements. Equation 6.0.5 shows the prediction of $-54.5 \mathrm{ppm} /{ }^{\circ} \mathrm{C}$ TCF, assuming the ideal calculated TCL $(+128$ $\mathrm{ppm} /{ }^{\circ} \mathrm{C}$ ) and $-750 \mathrm{ppm} /{ }^{\circ} \mathrm{C}$ for the $\mathrm{TC}$ of the $56 \mathrm{pF}$ capacitor. A temperature sweep was performed in the temperature chamber to measure the TCF, using the tank components in Table 6.2. Figure 6.0.11 shows the measured TCF of $+85 \mathrm{ppm} /{ }^{\circ} \mathrm{C}$.

Table 6.2: Tank components for fitting the mathematical model. NP0 - $0 \mathrm{ppm} /{ }^{\circ} \mathrm{C}$ and $\mathrm{SM}$ for silver mica, Figure 6.0.11

\begin{tabular}{|c|c|c|c|c|}
\hline QTY & Cap fixed & Cap Comp & Cvar & Inductor \\
\hline 1 & $100 \mathrm{p}$ SM & $56 \mathrm{p} \mathrm{N750}$ & $1.6 \mathrm{p}-11.2 \mathrm{p}$ & $11.3 \mu$ \\
\hline 5 & $10 \mathrm{p} \mathrm{NP0}$ & & & \\
\hline
\end{tabular}

$$
\begin{aligned}
T C F & =-\frac{1}{2}\left[128+(-750) \frac{56 p}{177.2 p}\right] \\
& =-54.5 \frac{\mathrm{ppm}}{{ }^{\circ} \mathrm{C}}
\end{aligned}
$$




$$
\begin{aligned}
T C F & =-\frac{1}{2}\left[150+(-850)_{N 750} \frac{56 p}{177.2 p}+(-15)_{N P 0} \frac{10 p}{177.2 p}+(-90)_{S M} \frac{100 p}{177.2 p}\right] \\
& =+85 \frac{p p m}{{ }^{\circ} C}
\end{aligned}
$$

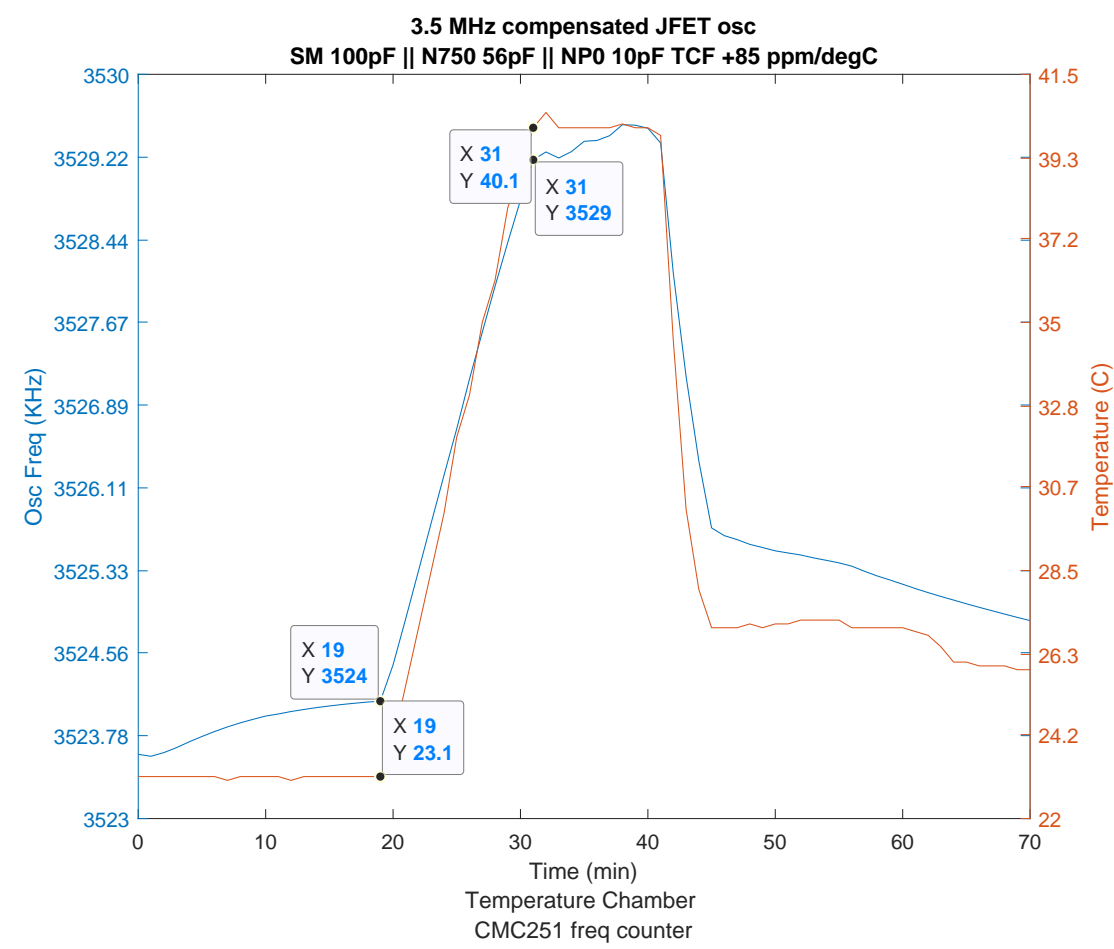

Figure 6.0.11: Overcompensation with 56 pF N750 compensating capacitor.

The measured $+85 \mathrm{ppm} /{ }^{\circ} \mathrm{C}$ was used to introduce some component non-idealities into the mathematical model of Equation 6.0.5, in order to match theory to measurements. Silver mica (SM) capacitors typically have some nonzero temperature coefficient, (roughly $-50 \mathrm{ppm} /{ }^{\circ} \mathrm{C}$ ), therefore the TCL was calculated accounting for approximate non-idealities, Equation 6.0.3. The range of TCL values was calculated to be about $-128 \mathrm{ppm} /{ }^{\circ} \mathrm{C}$ to -171 $\mathrm{ppm} /{ }^{\circ} \mathrm{C}$. The mean of these two values $\left(+150 \mathrm{ppm} /{ }^{\circ} \mathrm{C}\right)$ was used for subsequent TC calculations and TCF predictions. N750 capacitors have a TC tolerance of $+/-120 \mathrm{ppm} /{ }^{\circ} \mathrm{C}$, which provides a temperature coefficient range of $-630 \mathrm{ppm} /{ }^{\circ} \mathrm{C}$ to $-870 \mathrm{ppm} /{ }^{\circ} \mathrm{C}$. To fit the measured $+85 \mathrm{ppm} /{ }^{\circ} \mathrm{C}$ TCF to the model, the mean TCL of $+150 \mathrm{ppm} /{ }^{\circ} \mathrm{C}$ was used, 
and the N750 was adjusted to $-850 \mathrm{ppm} /{ }^{\circ} \mathrm{C}$ - close to its maximum. Adjusting the NP0 to $-15 \mathrm{ppm} /{ }^{\circ} \mathrm{C}$ for the $10 \mathrm{pF}$ capacitor, and the $\mathrm{SM} 100 \mathrm{pF}$ to $-90 \mathrm{ppm} /{ }^{\circ} \mathrm{C}$ in Equation 6.0.6, calculated to fit the measured result quite well. Therefore, $-90 \mathrm{ppm} /{ }^{\circ} \mathrm{C}$ and -15 $\mathrm{ppm} /{ }^{\circ} \mathrm{C}$ were used for subsequent TC calculations and TCF predictions for SM and NP0 parts, respectively.

$$
\begin{aligned}
T C F & =-\frac{1}{2}\left[T C L+T C_{C_{1}} \frac{C_{1}}{C_{t o t}}+T C_{C_{2}} \frac{C_{2}}{C_{t o t}}+T C_{C_{3}} \frac{C_{3}}{C_{t o t}}\right] \\
& =-\frac{1}{2}\left[150+(-470) \frac{36.8 p}{180.5 p}+(-15) \frac{30 p}{180.5 p}+(-90) \frac{101 p}{180.5 p}\right] \\
& =+0.6 \frac{p p m}{{ }^{\circ} C} \\
T C F_{\text {high }} & =-\frac{1}{2}\left[150+\mathbf{( - 4 1 0 )} \frac{36.8 p}{180.5 p}+(-15) \frac{30 p}{180.5 p}+(-90) \frac{101 p}{180.5 p}\right] \\
& =+6.7 \frac{p p m}{{ }^{\circ} C} \\
T C F_{\text {low }} & =-\frac{1}{2}\left[150+\mathbf{( - 5 3 0 )} \frac{36.8 p}{180.5 p}+(-15) \frac{30 p}{180.5 p}+(-90) \frac{101 p}{180.5 p}\right] \\
& =-5.5 \frac{p p m}{{ }^{\circ} C}
\end{aligned}
$$

Table 6.3: 7.05 - 7.22 MHz tank components, NP0 - $0 \mathrm{ppm} /{ }^{\circ} \mathrm{C}$ and SM for silver mica. Figures 6.0.13 and 6.0.12, and Equation 6.0.7

\begin{tabular}{|c|c|c|c|c|}
\hline QTY & Cap fixed & Cap Comp & Cvar & Inductor \\
\hline 1 & $101 \mathrm{p}$ SM & $36.8 \mathrm{p} \mathrm{N} 470$ & $1.6 \mathrm{p}-11.2 \mathrm{p}$ & $11.3 \mu$ \\
\hline 3 & $10.5 \mathrm{p}$ NP0 & & & \\
\hline
\end{tabular}

Temperature stability was achieved at $-1.42 \mathrm{ppm} /{ }^{\circ} \mathrm{C}$ for frequency range $7.05-7.22$ $\mathrm{MHz}$ with styrofoam packaging placed on the inductor and the capacitors for thermal insulation. Without insulation, $-4.25 \mathrm{ppm} /{ }^{\circ} \mathrm{C}$ was measured. The temperature compensating part was a single $36 \mathrm{pF}$ capacitor with temperature coefficient of N470, meaning -470 


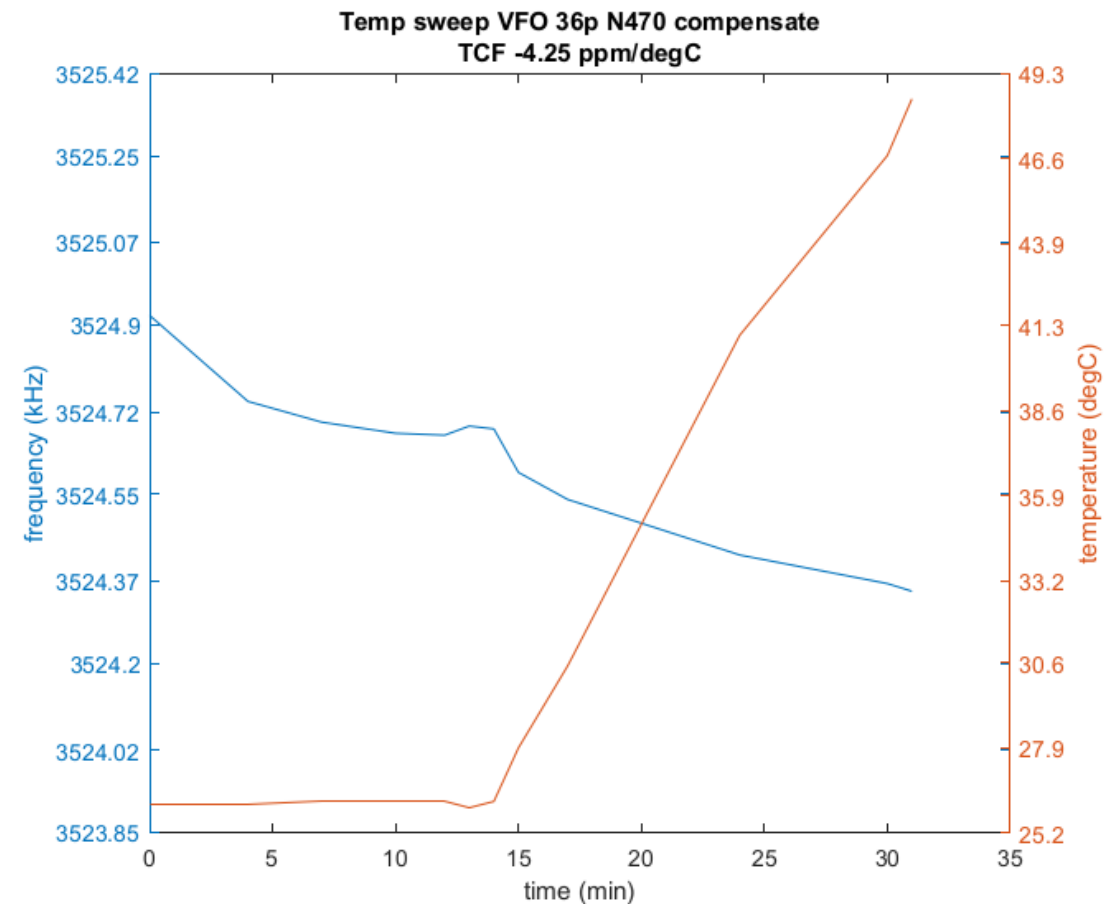

Figure 6.0.12: VFO drift with temperature compensating capacitor and low, (zero) temp-co capacitors for frequency doubled tuning range 7.05- 7.22 MHz, (doubled frequency).

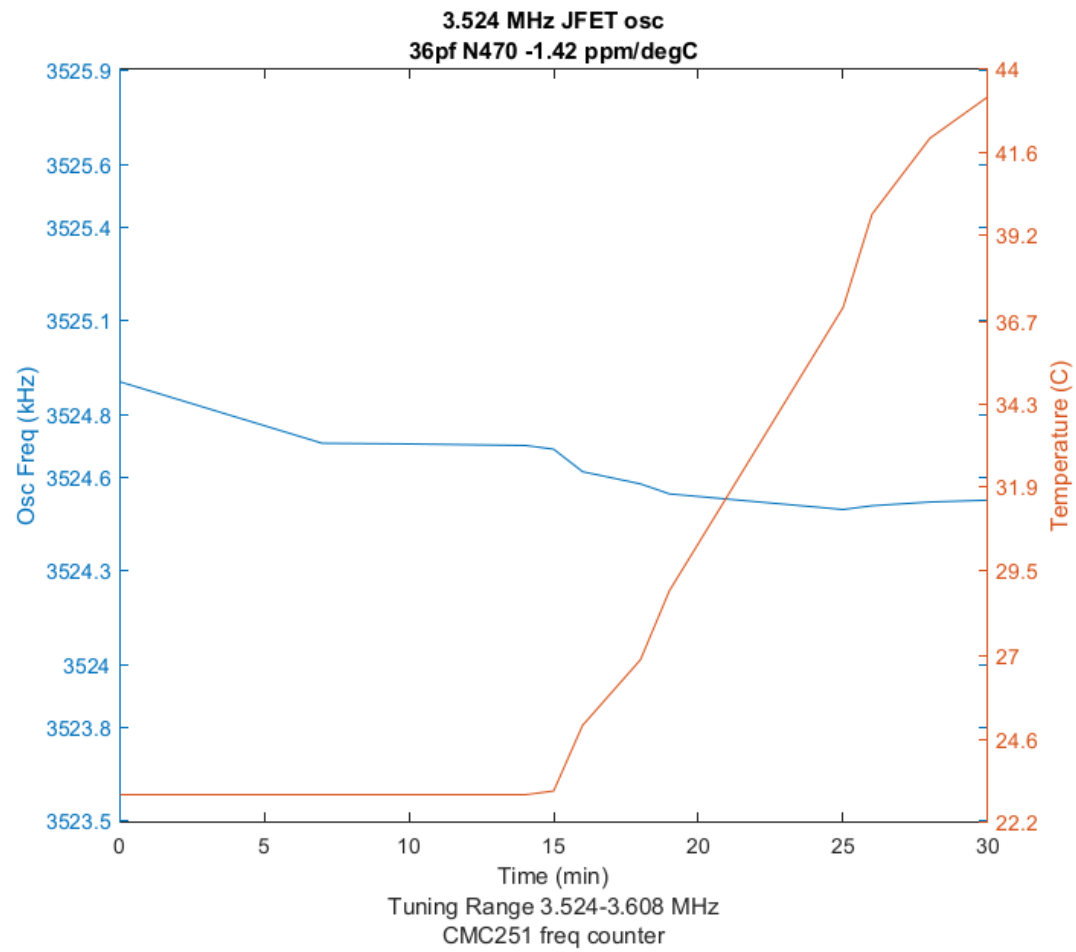

Figure 6.0.13: Temperature drift of 7.05 - 7.22 MHz, (doubled frequency), tuned oscillator with styrofoam packaging on tank inductor and capacitors, $-1.42 \mathrm{ppm} /{ }^{\circ} \mathrm{C}$. 


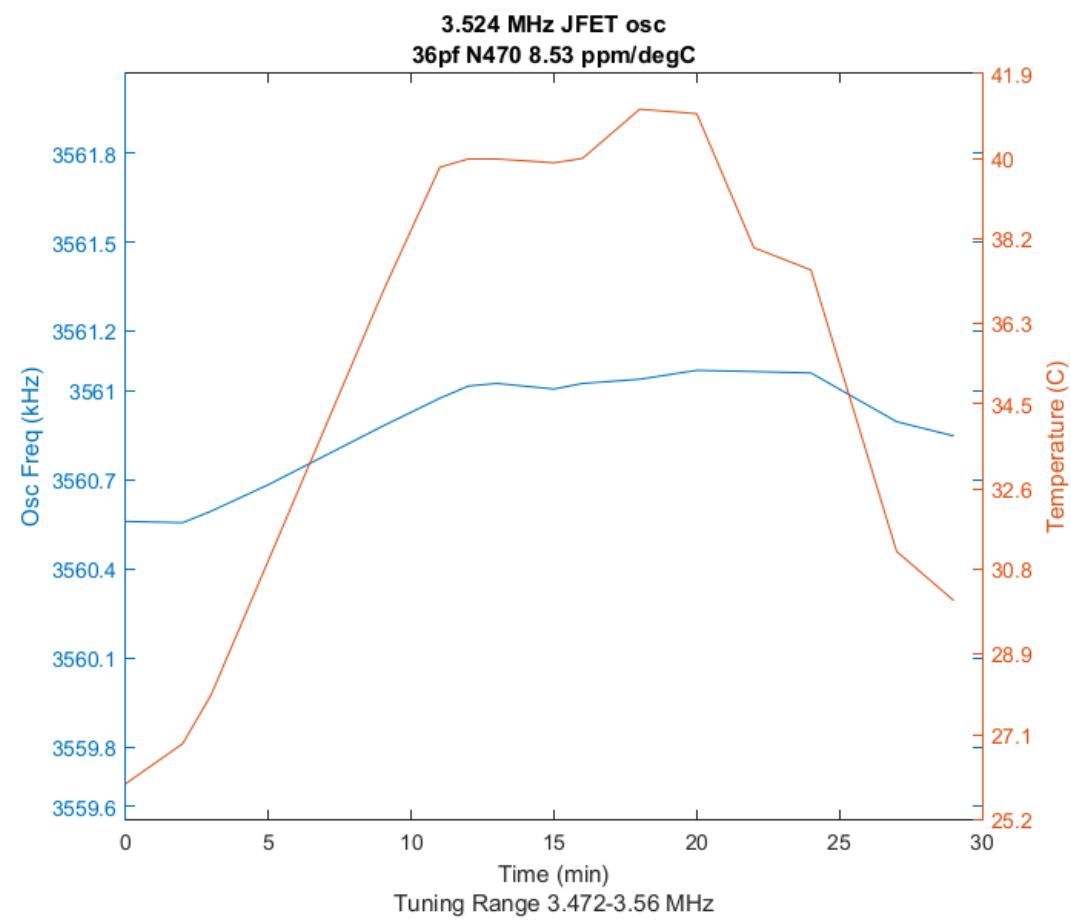

Figure 6.0.14: VFO drift with temperature compensating capacitor and low, (zero) temp-co capacitors for frequency doubled tuning range $6.95-7.13 \mathrm{MHz}$, (doubled frequency).

$\mathrm{ppm} /{ }^{\circ} \mathrm{C}$, Table 6.3. The predicted TCF with this compensating capacitor was $+0.6 \mathrm{ppm} /{ }^{\circ} \mathrm{C}$, Equation 6.0.7, where nominal $-470 \mathrm{ppm} /{ }^{\circ} \mathrm{C} \mathrm{TC}$ was assumed. Non-idealities of the three $10.5 \mathrm{pF}$ NP0 parts were assumed to have $-15 \mathrm{ppm} /{ }^{\circ} \mathrm{C}$ temperature coefficient. The $101 \mathrm{pF}$ assumed to have $-90 \mathrm{ppm} /{ }^{\circ} \mathrm{C}$ temperature coefficient. These non-idealities were informed from the previous heuristic measurement. This was quite close to the measured -4.25 $\mathrm{ppm} /{ }^{\circ} \mathrm{C} \mathrm{TCF}$ and within the range of tolerance of the $\mathrm{N} 470 \mathrm{TC}--470+/-60 \mathrm{ppm} /{ }^{\circ} \mathrm{C}-$ Equation 6.0.8. Temperature stability was also tested for the frequency range $6.95-7.13$ $\mathrm{MHz}$, which yielded $+8.5 \mathrm{ppm} /{ }^{\circ} \mathrm{C}$, Figure 6.0.14. The components used for this test are listed in Table 6.4.

Table 6.4: 6.95 - 7.13 MHz tank components, $\mathrm{NP0}-0 \mathrm{ppm} /{ }^{\circ} \mathrm{C}$ and SM for silver mica. Figure 6.0.14

\begin{tabular}{|c|c|c|c|c|}
\hline QTY & Cap fixed & Cap Comp & Cvar & Inductor \\
\hline 1 & $85.3 p$ SM & $36.8 p$ N470 & $1.6 \mathrm{p}-11.2 \mathrm{p}$ & $11.3 \mu$ \\
\hline 5 & $10.5 \mathrm{p} \mathrm{NP0}$ & & & \\
\hline
\end{tabular}


It is quite a practical challenge to procure stable and specific temperature coefficient capacitors these days. None could be found in leaded form from Digikey or Mouser. All of the temperature coefficient and stable capacitors were purchased and scavenged from SurplusGizmos or received from the author's professor. The ARRL chapter on Oscillators [16] notes that the supply of such capacitors is disappearing due to frequency synthesizers becoming the standard. 


\section{Chapter 7 \\ Conclusion and Future Work}

A frequency stabilization technique of Wes Hayward's [14] to stabilize frequency drift with temperature of a JFET Hartley VFO has been demonstrated. The requisite temperature chamber to perform the temperature drift measurements was constructed by the author in accordance with suggestions of Wes Hayward's article [14], discussed in chapter 6 of this work. This work represents the first known presentation of careful measurements of the improvement in initial and long term frequency drift resulting from immersion of the entire VFO circuit in boiling water. The VFO frequency drift with temperature was compensated to $-4.25 \mathrm{ppm} /{ }^{\circ} \mathrm{C}$ and $+8.5 \mathrm{ppm} /{ }^{\circ} \mathrm{C}$ for the $40 \mathrm{~m}$ frequency ranges: $7.05-7.22 \mathrm{MHz}$, and $6.95-7.13 \mathrm{MHz}$, respectively. The frequency drift was further reduced with styrofoam packaging on the tank components, achieving $-1.42 \mathrm{ppm} /{ }^{\circ} \mathrm{C}$. The theoretical frequency drift model was also heuristically modified to fit measured results to enable more accurate temperature drift of frequency (TCF) predictions. The resultant adjusted model, Equation 6.0.7, predicted $+0.6 \mathrm{ppm} /{ }^{\circ} \mathrm{C}$ for the $7.05-7.22 \mathrm{MHz}$ compensated $\mathrm{VFO}$, (using the nominal TC of the compensating capacitor), which measured at $-4.25 \mathrm{ppm} /{ }^{\circ} \mathrm{C}$. The measured TCF was within the tolerance of the compensating component's temperature coefficient, which predicted a TCF in the range of -6.7 to $+5.5 \mathrm{ppm} /{ }^{\circ} \mathrm{C}$, Equation 6.0.8.

Also shown in this work was the solution of the iqx2 [1] with opposite sideband suppression sensitivity to LO drive-level. A fixed-level VFO achieved $40 \mathrm{~dB}+$ opposite sideband suppression of the iqx 2 for $\mathrm{LO}$ drive-levels in the range of $+2.25-+5 \mathrm{dBm}$. Measurement of the phase noise of the JFET Hartley VFO in this thesis using an available instrument during the author's internship at Rohde \& Schwarz confirmed that this topology is capable of remarkable performance, significantly better than the signal generators and measurement instruments in the Portland State laboratories. The phase noise of the VFO 
was measured and found to be $-150 \mathrm{dBc} / \mathrm{Hz}$, which was in accordance with the literature on this VFO topology [4] [22].

When the VFO was frequency stabilized and applied back to measuring the opposite sideband suppression of the iqx 2 mixer module, more design improvements were made to the VFO in order to improve opposite sideband suppression. It was discovered that some $50 \Omega$ attenuation was needed between the output of the VFO and the input of the iqx 2 to present a wideband $50 \Omega$ load, which improved the opposite sideband suppression to the $40 \mathrm{~dB}+$ results. Additionally, it was discovered through experimentation and review of literature [4] that the oscillator's output power should be varied by varying the power supply, not through varying the JFET gate coupling capacitor.

This work presents the measurements performed on the experimental iqx 2 circuit, the work done on temperature compensation of the VFO, and the fitting of the VFO into a working test setup for the iqx2. The method for frequency drift compensation was a principally simple one, but which has been expressed as tedious in literature [16]. The author would not attempt to discount such a claim, but notes that the tedium of the process was very useful especially for a new HAM radio operator. The experience, to use Dr. Campbell's phrase, "digging into the onion" and working all aspects of the experiment right down to building part of the test setup was extremely rewarding and revealing.

Considering the work that has been done here, a complete receiver has still not been acheived. Further blocks to complete this full direct conversion phasing receiver include an RF LNA and a low-pass input filter. Additionally, the IQ mixer module and baseband audio signal processor would be a project for the author to build; the one measured here is not the author's property. To use the half-frequency VFO described in this work, some improvements could be made to the interface of the iqx 2 . A $3 \mathrm{~dB}$ or $6 \mathrm{~dB} 50 \Omega$ pad at the output of the VFO would provide a nice wideband $50 \Omega$ termination to the LO frequency doubler port. Additionally, a low-pass filter would be useful to knock down oscillator 
harmonics, (which the 3rd harmonic is $24 \mathrm{~dB}$ down), Figure 4.0.4. According to EMRFD [4] even order harmonics can severely imbalance the balanced frequency doubler, Figure 3.0.1, and degrade the suppression of the fundamental feed-through. This might further compromise the balanced mixer if the fundamental and strong harmonics are both present at the LO input. It might be worth experimenting further with some filtering of the half frequency VFO output before the frequency doubler input. Although the second harmonic is about $28 \mathrm{~dB}$ down from the fundamental already, a 3-pole low-pass Chebyshev filter with cutoff frequency $5 \mathrm{MHz}$ would knock the 2 nd harmonic down to about $38 \mathrm{~dB}$ down from the fundamental. Whether the lower harmonic levels from use of the low pass has an improvement on balance in the frequency doubler and/or mixer could be approximated by observation of the effect on the opposite sideband suppression. An increase in opposite sideband suppression with use of the low pass filter with respect to without the low pass filter might lead to the conclusion that reducing harmonic energy at the frequency doubler input preserves balance of the frequency doubler and perhaps the mixer. All measurements with and of the VFO were done with the circuit open on the bench, so the natural next step would be to build the VFO in a shielded container, an Altoids can would be an ideal choice as it is the right size and readily available. Some foam packing could be easily installed for some temperature insulation which would further stabilize any temperature induced frequency drift. 


\section{References}

[1] Rick Campbell and Katlin Dahn, "Simple New Analog-RF Weak Signal Receivers" in Proceedings of the 2019 VHF Super Conference, Sterling, VA, April 2019, American Radio Relay League, ISBN:978-1-62595-102-1, pp. 196-203

[2] Richard Campbell, Portland State University, "HF-VHF-UHF IQ Mixer with a Single SPDT Switch” IEEE MTT-S IMS Conference Paper, May 2015.

[3] Richard Campbell, TriQuint Semiconductor "An Integrated I-Q Mixer for SoftwareDefined Radio Applications" High Frequency Design, Jan. 2004, pp 26-33.

[4] Wes Hayward, Rick Campbell, Bob Larkin, Experimental Methods in RF Design Newington, CT: American Radio Relay League, Inc, 2016 Classic Reprint. Chap. 5.

[5] Wes Hayward, Rick Campbell, Bob Larkin, Experimental Methods in RF Design Newington, CT: American Radio Relay League, Inc, 2016 Classic Reprint. Chap. 5 pp 5.8 .

[6] Wes Hayward, Rick Campbell, Bob Larkin, Experimental Methods in RF Design Newington, CT: American Radio Relay League, Inc, 2016 Classic Reprint. Chap. 9.

[7] Richard Campbell, Notes: "New Mixer Design Notes" April 26, 2007. [Online]. Available: http://web.cecs.pdx.edu/ campbell/mixnotes.pdf (Accessed on August 09, 2019)

[8] Behzad Razavi, RF Microelectronics 2nd Ed. London, UK: Pearson Education, Inc, 2012. Chap. 4.

[9] Rick Lyons, "Quadrature Signals: Complex but not Complicated" White Paper IEEE Long Island Section. [Online]. Available: https://www.ieee.li/pdf/essay/quadrature signals.pdf (Accessed on Jul. 28, 2019)

[10] Rick Lyons, 'Understanding the 'Phasing Method' of Single Sideband Demodulation" DSPrelated.com. Aug. 8, 2012 [Online]. Available: https:// www.dsprelated.com/showarticle/176.php (Accessed on August 30, 2019)

[11] Ralph Oppelt, "The Generation and Demodulation of SSB Signals Using the Phasing Method Part 1: Basic Theory" VHF Communications, vol. 19, ed 2, Summer 1987, pp 66-72.

[12] Ralph Oppelt, "The Generation and Demodulation of SSB Signals Using the Phasing Method Part 2: Signal Processing for SSB/DSB/AM Transceiver Without Using Crystal Filters" VHF Communications, vol. 19, ed 3, Fall 1987, pp 130-140. 
[13] Mike Arnold, Randy Class, Francesco Dantoni, Shrinivasan Jaganathan, and Roland Sperlich, Texas Instruments. Application Report SLWA05 "LO Harmonic Effects on I/Q Balance and Sideband Suppression in Complex I/Q Modulators" May 09, 2010. [Online]. Available: http://www.ti.com/lit/an/slwa059/slwa059.pdf (Accessed on Oct. 03, 2019)

[14] Wes Hayward, "Measuring and Compensating Oscillator Frequency Drift” QST, Dec. 1993, pp 37-41.

[15] Jacob Makhinson, ”A Drift-Free VFO” QST, Dec. 1996, pp 32-36.

[16] David Stockton, Frederick J. Telewski, ”Oscillators and Synthesizers" Newington, CT: ARRL Handbook 2012. Chap. 10.

[17] Wes Hayward, Private Correspondence Jul. 2019.

[18] Wes Hayward, Rick Campbell, Bob Larkin, Experimental Methods in RF Design Newington, CT: American Radio Relay League, Inc, 2016 Classic Reprint. Chap. 4.

[19] Richard Campbell, TriQuint Semiconductor, "LO Phase-Noise Management in Amateur Radio Receiver Systems" White Paper.

[20] Richard Campbell, "A Black Box HF-VHF Instrumentation Receiver" White Paper. [Online]. Available: http://web.cecs.pdx.edu/ campbell/iR2ch8.pdf (Accessed on August 09, 2019)

[21] Richard Campbell, "Direct-Conversion Receiver Noise Figure" QST Technical Correspondence, Feb. 1996, pp 82-84.

[22] Wes Hayward, Doug DeMaw Solid State Design for the Radio Amateur Newington, CT: American Radio Relay League, Inc, 1986. Chap. 3.

[23] Kay Green, Agilent Technologies, Inc, Presentation "Phase Noise Measurements Methods and Techniques" 2012. [Online]. Available: https://www.keysight.com/ upload/cmc_upload/All/PhaseNoise_webcast_19Jul12.pdf

[24] Rhode \& Schwarz, Application Note: "Phase Noise Measurements with Spectrum Analyzersof the FSE family" Dec. 08, 1995. [Online]. Available: https: //scdn.rohde-schwarz.com/ur/pws/dl_downloads/dl_application/application_notes/ 1epan16/1epan16e.pdf

[25] Richard Campbell, "High-Performance Direct-Conversion Receivers" QST, Aug. 1992, pp 19-28.

[26] Richard Campbell, "High-Performance, Single-Signal Direct-Conversion Receivers" QST, Jan. 1993, pp 32-40. 
[27] Richard Campbell, "The MicroR2 - An Easy to Build "Single Signal" SSB or CW Receiver", 4th ed., QST, Oct. 2006 pp 28-32.

[28] Roy Lewallen, ”An Optimized QRP Transceiver" QST, Aug. 1980, pp 14-19.

[29] Wes Hayward, Dick Bingham, "Direct Conversion: A Neglected Technique" QST, Nov. 1968, pp 15-17.

[30] Wes Hayward, Rick Campbell, Bob Larkin, Experimental Methods in RF Design Newington, CT: American Radio Relay League, Inc, 2016 Classic Reprint. Chap. 8.

[31] Joel Hallas, "Receivers" Newington, CT: ARRL Handbook 2012. Chap. 12.

[32] Richard Campbell, Notes: "Medium Power Diode Frequency Doublers". May 20, 1999. [Online]. Available: http://web.cecs.pdx.edu/ campbell/mixnotes.pdf (Accessed on Jul. 09, 2019)

[33] Stephen A. Maas, "A GaAs MESFET Mixer with Very Low Intermodulation" IEEE Transactions on Microwave Theory and Techniques, vol. MTT-35, no. 4, Apr. 1987, pp 425-429.

[34] Richard Campbell, Cascade Microtech, "High Frequency Differential Passive FET Direct Conversion Mixer/Modulator” IEEE MTT-S IMS Digest 2006, pp 922-925.

[35] Richard Campbell, "Microwave Downconverter and Upconverter Update" White Paper.

[36] Wes Hayward, personal website. "Two Faces of Q" Nov. 2010. [Online]. Available: http://w7zoi.net/twofaces.pdf (Accessed on Jul. 06, 2019).

[37] R. Fischer, "Broadband Twisted-Wire Quadrature Hybrids" IEEE Transactions on Microwave Theory and Techniques, vol. 21, no. 5, May 1973, pp 355-357.

[38] D. Pozar, Microwave Engineering, 4th ed. Hoboken, NJ: John Wiley and Sons, Inc, 2012.

[39] Kenneth K. Kaiser, Kettering University, Electromagnetic Compatibility Handbook Flint, MI: Taylor and Francis Group, Sept. 2004.

[40] H. T. Friis, FELLOW, I.R.E. "Noise Figures of Radio Receivers" Proceedings of the I.R.E. Jul. 1944.

[41] Agilent Technologies, Inc, "Agilent 8648A/B/C/D Signal Generators Data Sheet" Nov. 6, 2006. pp 2. [Online]. Available: https://literature.cdn.keysight.com/litweb/ pdf/5965-3432E.pdf?id=1000031128:epsg:dow (Accessed Jul. 9, 2019) 
[42] Tektronix, Inc, RSA3300A Series, "Real Time Spectrum Analyzers" Sep. 2006. pp 8. [Online]. Available: http://www.testequipmenthq.com/datasheets/TEKTRONIXRSA3303A-Datasheet.pdf (Accessed Jul. 09, 2019)

[43] Darioush Agahi, William Domino, Nooshin Vakilian, "Two-tone vs. Single-tone Measurement of Second-order Nonlinearity" Microwave Journal, Mar. 1, 2002. [Online]. Available: https://www.microwavejournal.com/articles/print/3411-two-tonevs-single-tone-measurement-of-second-order-nonlinearity (Accessed Oct. 07, 2019).

[44] Doug Grant, Business Development Director, RF \& Wireless Systems, Analog Devices, "Solving the Direct Conversion Problem" Wilmington, MA: EE Times, Dec. 09, 2000. [Online]. Available: https://www.eetimes.com/document.asp?doc_id= 1224754\# (Accessed Oct. 07, 2019).

[45] Doug Stuetzle, Linear Technology, "Understanding IP2 and IP3 Issues in Direct Conversion Receivers for WCDMA Wide Area Basestations" Analog Devices Technical Article. [Online]. Available: https://www.analog.com/en/technical-articles/ understanding-ip2-and-ip3-issues-in-direct-conversion-receivers.html (Accessed Oct. 07, 2019)

[46] Bill Foose NK1Z, "High Frequency Communications - An Introductory Overview - Who, What, and Why?" Aug. 13, 2012. [Online]. Available: http://pcars.org/ PROGRAMS/Foose_Bill\%20Eweek.pdf (Accessed Jun. 6, 2019).

[47] Gordon McComb, "Understanding Capacitor Markings" Robot Builder's Bonanza Application Notes. 2011. [Online]. Available: http://www.robotoid.com/appnotes/ electronics-capacitor-markings.html (Accessed Jun. 21, 2019). 


\section{Appendix A \\ Mixer Mathematics}

A barrage of excellent sources conspired to permit the following summary of Hartley IQ image rejection, [6] [8] [9] [11].

\section{A.1 Terminology}

The terminology of in-phase (I) and quadrature (Q) signifies a $90^{\circ}$ phase difference between I and Q with the $90^{\circ}$ being attributed to the Q signal. The term quadrature will be used as a verb meaning to apply a $90^{\circ}$ phase-shift, and as a noun meaning the $90^{\circ}$ phaseshift itself. The upper sideband (USB) will be treated first with the lower sideband (LSB) being summarized as a dual of the USB case. In the present treatment the USB is favored with low-side injection of the local oscillator (LO) meaning that the $\mathrm{LO}$ frequency is lower than the desired RF signal. The LSB is considered to be the image signal located equidistant in frequency from the LO as the USB, but lower than the LO. Hereafter "signal" and "USB" will be used interchangeably; likewise "image" and "LSB" will be interchangeable. To reverse the subsequent analysis in favor of the LSB, the Q channel may be inverted and the USB would then cancel. This can be visualized in Figure A.8.4 where the lower left plot Q signal and image would be inverted summing with I to suppress the signal. In this case the "image" would be the signal and the "signal" would be the image and this would be considered high-side LO injection.

\section{A.2 Baseband I \& Q Output}

To mathematically exemplify IQ image-reject mixing, the time-domain is more suitable than the frequency-domain analogue. As such the time-domain basic representations of 
band-limited signals are used in Equations A.5.3 and A.5.4 for the RF and LO signals, respectively.

In an IQ system, quadrature can be applied to the LO or to the RF input. In the present example, quadrature is applied to the LO. For convenience, the LO signal amplitude has been arbitrarily set to 2 to cancel with the $\frac{1}{2}$ coefficient of Equation A.7.1 resulting from multiplication of the RF and LO signals. Because the image, LSB, and the desired signal, USB, are spaced equally in frequency from the LO frequency, they translate to the same frequency by the trigonometric property in Equations A.7.2 and A.7.3; note Equation A.7.3 applies a sign inversion, but the phase argument is the same.

The heterodyne principle employs a mixer to multiply the input RF signal at $\omega_{R F}$ frequency by the input LO at $\omega_{L O}$ frequency to produce the intermediate frequency (IF) at the output, where frequency has been defined by Equation A.5.1 in $\left(\frac{\mathrm{rad}}{\mathrm{s}}\right)$. The signal multiplication follows the trigonometric identity in Equation A.7.1. As indicated by the trig identity of A.7.1, sum and difference products result from RF and LO signal multiplication; Equations A.5.5 and A.5.11 demonstrate this. The difference product is desired for down-conversion in receivers and a low-pass filter removes the sum product, leaving the difference term in Equations A.5.7 and A.5.11; this is the IF. The IF may be from zero Hz up to the cutoff frequency of the low-pass filter. This frequency range is considered baseband. Thus the low-passed IF outputs for USB and LSB I and Q signals are repersented by $b b_{i / q}{ }^{\prime}(t)$ and $b b_{i / q}{ }^{\prime \prime}(t)$, respectively, 'bb' for baseband.

\section{A.3 IQ Sum to Reject LSB}

The Q baseband signal is subjected to an additional $-90^{\circ}$ phase shift, Equation A.5.13, before being summed with the I baseband signal to give the USB output, Equation A.5.15. In conjuction with the USB signal path, the LSB is present and treated similarly resulting in the LSB output, Equation A.6.6. 


\section{A.4 Error Terms}

Important assumptions and approximations were made to achieve the results of Equations A.5.15b and A.6.6b. The coefficients and terms $\epsilon$ and $\delta$ are amplitude and phase error terms, respectively. They account for imperfections in the I and Q signal paths from when they are split, converted, phase-shifted and then summed and for the phase error from imperfections in generating the $\mathrm{LO}$ quadrature. The additional $-90^{\circ}$ phase shift is typically a network that applies a phase shift to both the I and Q channels such that the difference between them remains $90^{\circ}$. For this reason the phase-shift network imparts a frequency dependence on the LO phase, $\phi_{o}(\omega)$, which appears in both the I and Q baseband signals. Similarly, the phase error term, $\delta(\omega)$, gains some frequency dependence through the phase-shift network and this shows up in the baseband Q signal. The Q baseband signal is separated into individual terms and the trigonometric identity A.7.6 is used to produce the 4 terms in A.5.14a. The $\delta$ and $\epsilon$ terms were taken to be smaller than 0.1, allowing for the small angle approximation, Equation A.7.7, to be made in Equation A.5.14b. The 4th term in A.5.14b is subsequently ignored, A.5.14c. The USB output, Equation A.5.15a, is comprised of the desired signal with an amplitude of 2 , and two error terms with amplitudes much less than 2 , so are therefore safely ignored. For the LSB output, it can be seen from Equation A.6.6b that only error terms remain. The smaller the phase and amplitude errors, the more effectively the LSB is rejected.

\section{A.5 Upper Sideband (USB) desired for low-side LO injection: $\omega_{R F}>\omega_{L O}$}

$$
\omega=\frac{1}{2 \pi f} \quad \frac{r a d}{s}
$$

$$
\text { define: } \omega_{I F}=\omega_{L O}-\omega_{R F}
$$




\section{USB In-Phase mixing:}

$$
\begin{gathered}
R F_{i}(t)=a_{R F}(t) \cos \left(\omega_{R F} t+\phi_{R F}(t)\right) \\
L O_{i}(t)=2 \cos \left(\omega_{L O} t+\phi_{o}\right) \\
R F_{i}(t) L O_{i}(t)=a_{R F}(t) \cos \left(\left(\omega_{R F}+\omega_{L O}\right) t+\phi_{R F}(t)+\phi_{o}\right) \\
+a_{R F}(t) \cos \left(\left(\omega_{R F}-\omega_{L O}\right) t-\phi_{R F}(t)+\phi_{o}\right) \\
\boldsymbol{L P F}\left\{R F_{i}(t) L O_{i}(t)\right\}=a_{R F}(t) \cos \left(\left(\omega_{R F}-\omega_{L O}\right) t-\phi_{R F}(t)+\phi_{o}\right) \\
b b_{i}(t)=a_{R F}(t) \cos \left(-\omega_{I F} t-\phi_{R F}(t)+\phi_{o}\right) \\
=a_{R F}(t) \cos \left(\omega_{I F} t+\phi_{R F}(t)-\phi_{o}\right) \\
b b_{i}{ }^{\prime}(t)=a_{R F}(t) \cos \left(\omega_{I F} t+\phi_{R F}(t)-\phi_{o}(\omega)\right)
\end{gathered}
$$

\section{USB Quadrature mixing:}

$$
\begin{gathered}
R F_{q}(t)=[1+\epsilon] a_{R F}(t) \cos \left(\omega_{R F} t+\phi_{R F}(t)\right) \\
L O_{q}(t)=2 \cos \left(\omega_{L O} t+\phi_{o}-90^{\circ}+\delta\right)
\end{gathered}
$$

$$
\begin{aligned}
R F_{q}(t) L O_{q}(t) & =[1+\epsilon] a_{R F}(t) \cos \left(\left(\omega_{R F}+\omega_{L O}\right) t+\phi_{R F}(t)+\phi_{o}-90^{\circ}+\delta\right) \\
& +[1+\epsilon] a_{R F}(t) \cos \left(\left(\omega_{R F}-\omega_{L O}\right) t-\phi_{R F}(t)+\phi_{o}-90^{\circ}+\delta\right)
\end{aligned}
$$

$$
\begin{aligned}
b b_{q}(t) & =\boldsymbol{L P F}\left\{R F_{q}(t) L O_{q}(t)\right\} \\
& =[1+\epsilon] a_{R F}(t) \cos \left(\left(\omega_{R F}-\omega_{L O}\right) t-\phi_{R F}(t)+\phi_{o}-90^{\circ}+\delta\right) \\
& =[1+\epsilon] a_{R F}(t) \cos \left(-\omega_{I F} t-\phi_{R F}(t)+\phi_{o}-90^{\circ}+\delta\right) \\
& =[1+\epsilon] a_{R F}(t) \cos \left(\omega_{I F} t+\phi_{R F}(t)-\phi_{o}+90^{\circ}-\delta\right)
\end{aligned}
$$

$$
\begin{aligned}
b b_{q}{ }^{\prime}(t) & =[1+\epsilon(\omega)] a_{R F}(t) \cos \left(\omega_{I F} t+\phi_{R F}(t)-\phi_{o}(\omega)+90^{\circ}-90^{\circ}-\delta(\omega)\right) \\
& =[1+\epsilon(\omega)] a_{R F}(t) \cos \left(\omega_{I F} t+\phi_{R F}(t)-\phi_{o}(\omega)-\delta(\omega)\right)
\end{aligned}
$$




$$
\begin{aligned}
& b b_{q}{ }^{\prime}(t)=a_{R F}(t) \cos \left(\omega_{I F} t+\phi_{R F}(t)-\phi_{o}(\omega)\right) \cos (\delta(\omega)) \\
& -a_{R F}(t) \sin \left(\omega_{I F} t+\phi_{R F}(t)-\phi_{o}(\omega)\right) \sin (\delta(\omega)) \\
& +\epsilon(\omega) a_{R F}(t) \cos \left(\omega_{I F} t+\phi_{R F}(t)-\phi_{o}(\omega)\right) \cos (\delta(\omega)) \\
& -\epsilon(\omega) a_{R F}(t) \sin \left(\omega_{I F} t+\phi_{R F}(t)-\phi_{o}(\omega)\right) \sin (\delta(\omega)) \\
& =a_{R F}(t) \cos \left(\omega_{I F} t+\phi_{R F}(t)-\phi_{o}(\omega)\right) \\
& -\delta(\omega) a_{R F}(t) \sin \left(\omega_{I F} t+\phi_{R F}(t)-\phi_{o}(\omega)\right) \\
& +\epsilon(\omega) a_{R F}(t) \cos \left(\omega_{I F} t+\phi_{R F}(t)-\phi_{o}(\omega)\right) \\
& -\epsilon(\omega) \delta(\omega) a_{R F}(t) \sin \left(\omega_{I F} t+\phi_{R F}(t)-\phi_{o}(\omega)\right) \\
& =a_{R F}(t) \cos \left(\omega_{I F} t+\phi_{R F}(t)-\phi_{o}(\omega)\right) \\
& -\delta(\omega) a_{R F}(t) \sin \left(\omega_{I F} t+\phi_{R F}(t)-\phi_{o}(\omega)\right) \\
& +\epsilon(\omega) a_{R F}(t) \cos \left(\omega_{I F} t+\phi_{R F}(t)-\phi_{o}(\omega)\right) \\
& U S B_{\text {out }}=b b_{q}{ }^{\prime}(t)+b b_{i}{ }^{\prime}(t) \\
& =2 a_{R F}(t) \cos \left(\omega_{I F} t+\phi_{R F}(t)-\phi_{o}(\omega)\right) \\
& -\delta(\omega) a_{R F}(t) \sin \left(\omega_{I F} t+\phi_{R F}(t)-\phi_{o}(\omega)\right) \\
& +\epsilon(\omega) a_{R F}(t) \cos \left(\omega_{I F} t+\phi_{R F}(t)-\phi_{o}(\omega)\right) \\
& =2 a_{R F}(t) \cos \left(\omega_{I F} t+\phi_{R F}(t)-\phi_{o}(\omega)\right)
\end{aligned}
$$

A.6 Lower Sideband (LSB) image for low-side LO injection: $\omega_{R F}<\omega_{L O}$

\section{LSB In-Phase mixing:}

$$
\begin{gathered}
b b_{i}{ }^{\prime \prime}(t)=a_{R F}(t) \cos \left(\omega_{I F} t-\phi_{R F}(t)+\phi_{o}\right) \\
b b_{i}{ }^{\prime \prime}(t)=a_{R F}(t) \cos \left(\omega_{I F} t-\phi_{R F}(t)+\phi_{o}(\omega)\right)
\end{gathered}
$$

\section{LSB Quadrature mixing:}




$$
\begin{aligned}
& b b_{q}{ }^{\prime \prime}(t)=[1+\epsilon] a_{R F}(t) \cos \left(\omega_{I F} t-\phi_{R F}(t)+\phi_{o}-90^{\circ}+\delta\right) \\
& b b_{q}{ }^{\prime \prime}(t)=[1+\epsilon] a_{R F}(t) \cos \left(\omega_{I F} t-\phi_{R F}(t)+\phi_{o}(\omega)-90^{\circ}-90^{\circ}+\delta(\omega)\right) \\
&=[1+\epsilon] a_{R F}(t) \cos \left(\omega_{I F} t-\phi_{R F}(t)+\phi_{o}(\omega)-180^{\circ}+\delta(\omega)\right) \\
&=-[1+\epsilon] a_{R F}(t) \cos \left(\omega_{I F} t-\phi_{R F}(t)+\phi_{o}(\omega)+\delta(\omega)\right) \\
& b b_{q}^{\prime \prime}(t)=-a_{R F}(t) \cos \left(\omega_{I F} t-\phi_{R F}(t)+\phi_{o}(\omega)\right) \\
&+\delta(\omega) a_{R F}(t) \sin \left(\omega_{I F} t-\phi_{R F}(t)-\phi_{o}(\omega)\right) \\
&-\epsilon(\omega) a_{R F}(t) \cos \left(\omega_{I F} t-\phi_{R F}(t)+\phi_{o}(\omega)\right) \\
& \quad b_{q}^{\prime \prime}(t)+b b_{i}^{\prime \prime}(t) \\
&=a_{R F}(t) \cos \left(\omega_{I F} t-\phi_{R F}(t)+\phi_{o}(\omega)\right) \\
&-a_{R F}(t) \cos \left(\omega_{I F} t-\phi_{R F}(t)+\phi_{o}(\omega)\right) \\
&+\delta(\omega) a_{R F}(t) \sin \left(\omega_{I F} t-\phi_{R F}(t)+\phi_{o}(\omega)\right) \\
&-\epsilon(\omega) a_{R F}(t) \cos \left(\omega_{I F} t-\phi_{R F}(t)+\phi_{o}(\omega)\right) \\
&+\delta(\omega) a_{R F}(t) \sin \left(\omega_{I F} t-\phi_{R F}(t)+\phi_{o}(\omega)\right) \\
&-\epsilon(\omega) a_{R F}(t) \cos \left(\omega_{I F} t-\phi_{R F}(t)+\phi_{o}(\omega)\right) \\
&
\end{aligned}
$$

\section{A.7 Trigonometric Identities}

$$
\begin{gathered}
\cos (a) \cos (b)=\frac{1}{2}(\cos (a+b)+\cos (a-b)) \\
\cos (b)=\cos (-b) \\
-\sin (a)=\sin (-a) \\
\cos \left(a-90^{\circ}\right)=\sin (a) \\
\cos \left(a-180^{\circ}\right)=-\cos (a) \\
\cos (a+b)=\cos (a) \cos (b)-\sin (a) \sin (b)
\end{gathered}
$$


small angle approx. $\alpha<=0.1$

$$
\begin{aligned}
& \sin (\alpha)=\sim \alpha \\
& \cos (\alpha)=\sim 1
\end{aligned}
$$

\section{A.8 IQ Image Rejection Spectra Diagrams}

The preceding pages of Equations describing IQ mixer image rejection are summarized illustratively in Figures A.8.1-A.8.4. Whereas the mathematical treatment of IQ image rejection lends itself more usefully to the time-domain, it is more visually instructive in the frequency domain. Thus the band-limited signals, images, and LO signals are portrayed as spectra and the time-domain multiplication corresponds to convolution in the frequency domain. The plots in Figures A.8.1-A.8.4 are meant to illustrate the image cancellation. Thus the amplitudes of the signals are not to scale, the $+1,-1,-j,+j$ labels on the axes are for distinguishing real and imaginary axes. For illustrative purposes ideal amplitude and phase matching across I and Q is assumed.

The desired signal is the signal at $\omega_{i n}$, the outermost signal in the upper-left plot in Figure A.8.1, and the image is at $\omega_{i m}$. When the image and the desired channel are mixed with the LO, they are frequency shifted to both sums, $\left(\omega_{i n}+\omega_{L O}\right) \&\left(\omega_{L O}+\omega_{i m}\right)$, and differences, $\left(\omega_{i n}-\omega_{L O}\right) \&\left(\omega_{L O}-\omega_{i m}\right)$. With low pass filtering, the sum-products are removed, (attenuated), leaving the remaining difference products.

In Figure A.8.1, the RF signal and its image, top-left, are convolved with the idealized in-phase LO signal, (cosine spectrum), lower-left, to produce the frequency-translated lowpassed spectra to the right. This Figure corresponds to Equations A.5.3-A.5.7 and A.6.1, this is the I output. In Figure A.8.2 the RF signal and its image are convolved with the idealized quadrature LO signal, (sine spectrum), to similary produce the frequency-translated low-passed spectra to the right. Equations A.5.9-A.5.12 and A.6.3 correspond to this Figure. This is the Q output. The Hilbert transform of the Q signal and image is demonstrated 

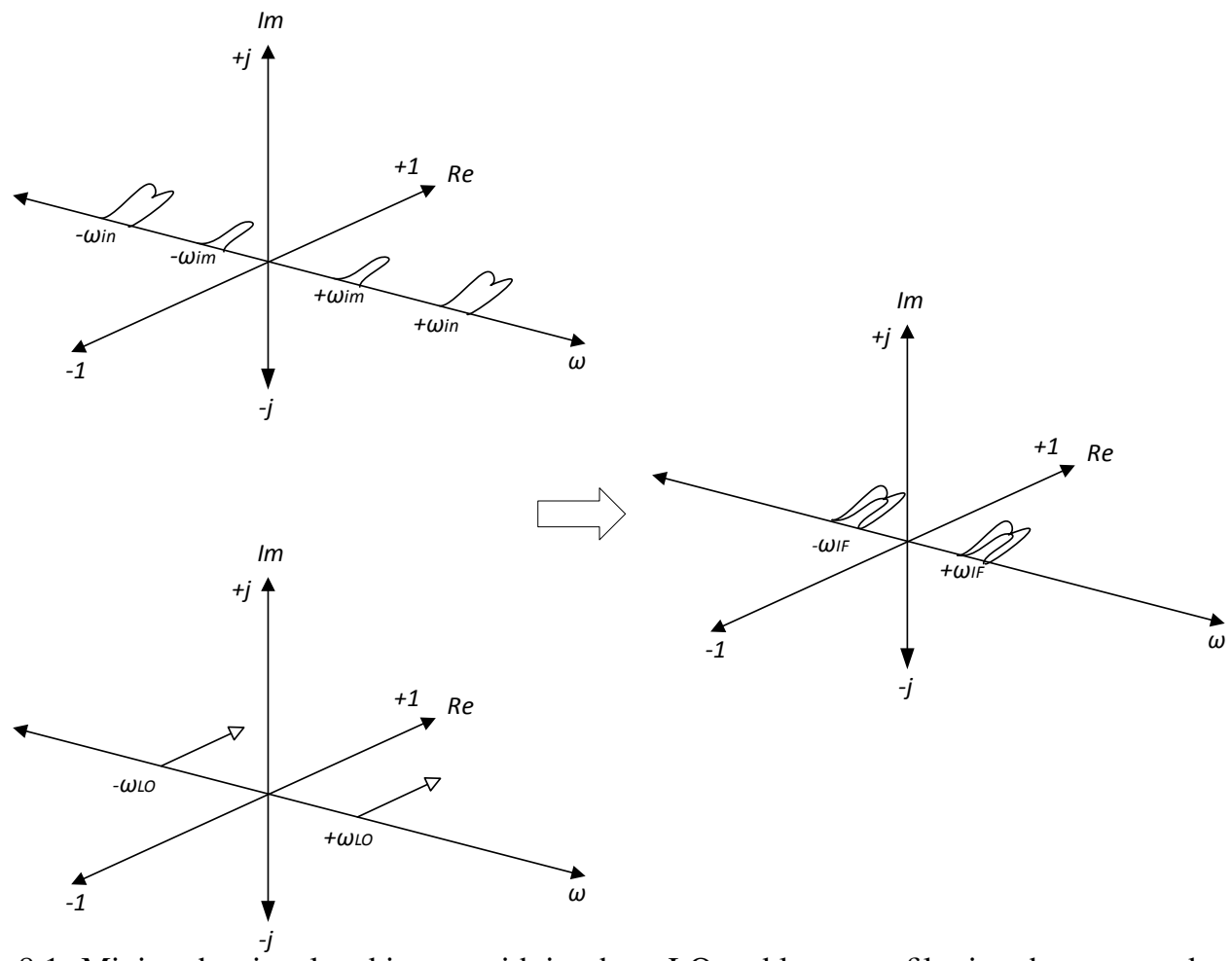

Figure A.8.1: Mixing the signal and image with in-phase LO and low pass filtering the sum product. This is the I output.

in Figure A.8.3 corresponding to Equations A.5.13 and A.6.4.

The final summation of the Hilbert transformed quadrature signal and image with the inphase signal and image is represented in Figure A.8.4 corresponding to Equations A.5.15b and A.6.6b. 

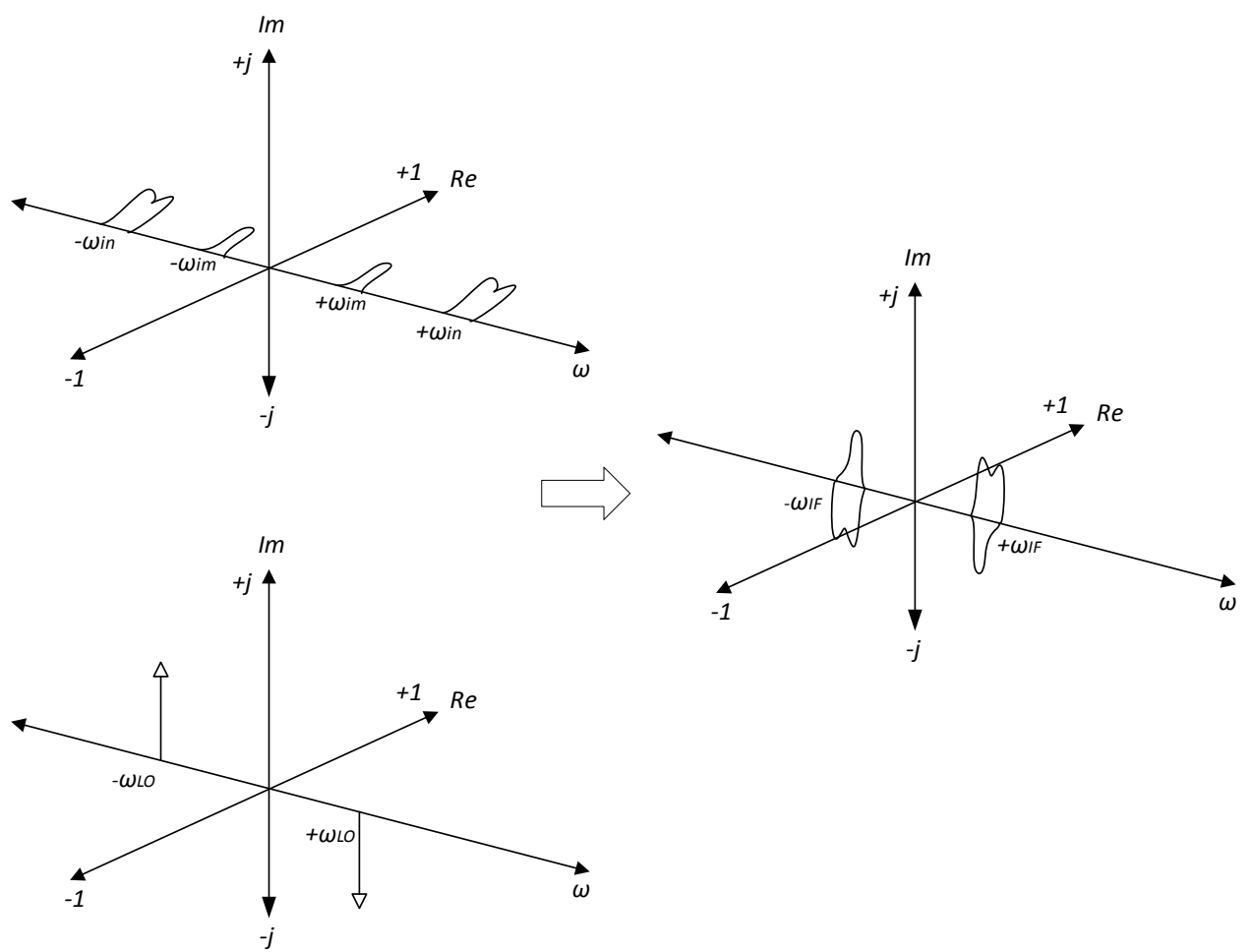

Figure A.8.2: Mixing the signal and image with quadrature LO results in the frequency shifted Hilbert transform of the signal and image with the signal inverted. Low pass filtering renders the $\mathrm{Q}$ output.

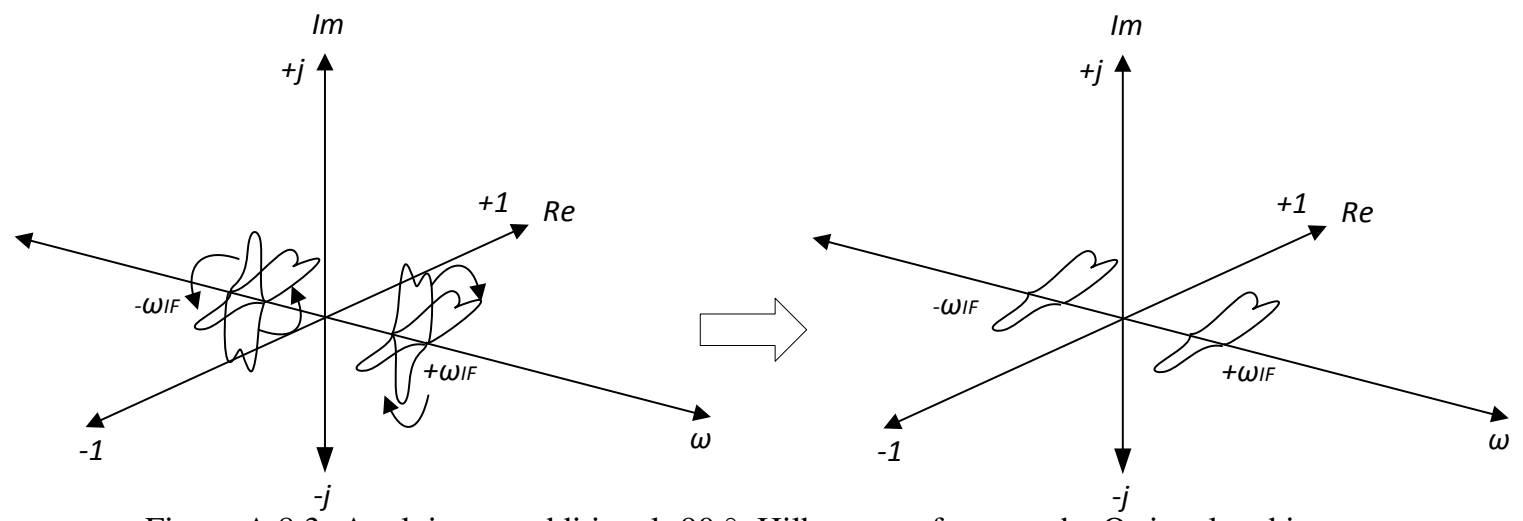

Figure A.8.3: Applying an additional $-90^{\circ}$, Hilbert transform, to the $\mathrm{Q}$ signal and image. 

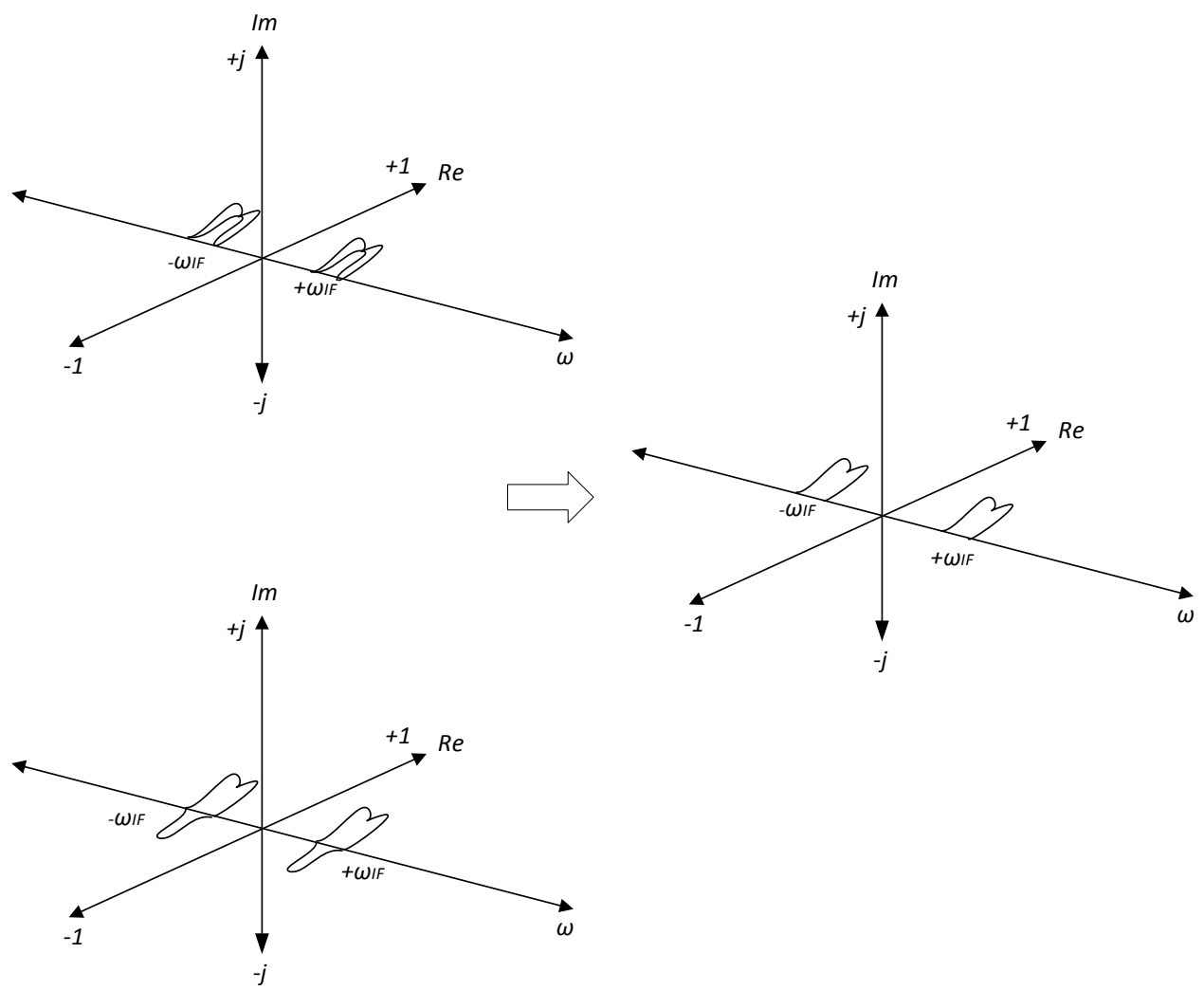

Figure A.8.4: The addition of the in-phase signal and image and Hilbert-transformed quadrature mixed signal and image results in the image being rejected. 


\section{Appendix B \\ Additional Receiver Background}

\section{"Cat's Whisker"}

The equivalent schematic with the familiar diode symbol is to the right in Figure B.0.1. The frequency is determined by the inductor, L, and the capacitor, C, see Equation B.0.2.
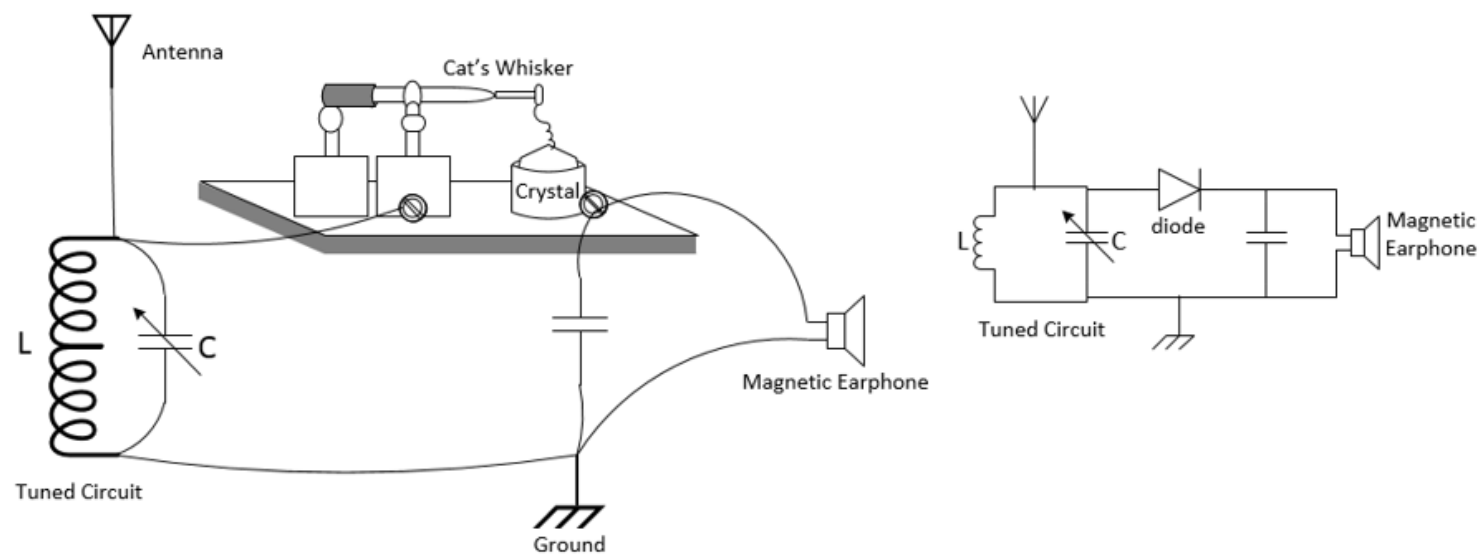

Figure B.0.1: Crystal TRF cat's whisker receiver for AM broadcast signals.

$$
\begin{gathered}
f=\frac{\omega}{2 \pi f} H z \\
\omega=\frac{1}{\sqrt{L C}} \frac{r a d}{s}
\end{gathered}
$$

